\title{
Direct CP Violation in $\boldsymbol{b} \rightarrow \boldsymbol{s} \boldsymbol{\gamma}$ Decays*
}

\author{
Andrew Eichenbaum \\ Stanford Linear Accelerator Center \\ Stanford University \\ Stanford, CA 94309
}

SLAC-Report-734

November 2004

\section{Prepared for the Department of Energy under contract number DE-AC02-76SF00515}

Printed in the United States of America. Available from the National Technical Information Service, U.S. Department of Commerce, 5285 Port Royal Road, Springfield, VA 22161.

\footnotetext{
*Ph.D. thesis University of Wisconsin - Madison
} 


\title{
Direct $C P$ Violation in $\boldsymbol{b} \rightarrow \boldsymbol{s} \boldsymbol{\gamma}$ Decays
}

\author{
by
}

\author{
ANDREW EICHENBAUM
}

A dissertation submitted in partial fulfillment of the requirements for the degree of

\section{Doctor of Philosophy}

(PHYSICS)

at the

UNIVERSITY OF WISCONSIN - MADISON

2004 


\title{
Direct $C P$ Violation in $\boldsymbol{b} \rightarrow \boldsymbol{s} \boldsymbol{\gamma}$ Decays
}

\author{
Andrew Eichenbaum \\ Under the supervision of Professor Richard Prepost \\ At the University of Wisconsin - Madison
}

This dissertation describes a measurement of the direct $C P$ asymmetry between inclusive $b \rightarrow s \gamma$ and $\bar{b} \rightarrow \bar{s} \gamma$ decays. This asymmetry is expected to be less than 0.01 in the Standard Model, but could be enhanced up to the order of 0.10 by non-Standard Model contributions. We use a sample of 89 million $B \bar{B}$ pairs recorded with the $B A B A R$ detector at PEP-II, from which we reconstruct a set of 12 exclusive $b \rightarrow s \gamma$ final states containing one charged or neutral kaon and one to three pions. We measure an asymmetry of $A_{C P}(b \rightarrow s \gamma)=$ $0.025 \pm 0.050$ (stat. $) \pm 0.015$ (syst.), corresponding to an allowed range of $-0.06<A_{C P}(b \rightarrow$ $s \gamma)<+0.11$ at $90 \%$ confidence level. 
For all the people who put up with me for the past 6 years. 


\section{Contents}

1 Abstract $\quad$ xviii

2 Introduction $\quad 1$

2.1 The Standard Model . . . . . . . . . . . . . . . . . . . 2

2.2 Supersymmetric Extensions to the Standard Model . . . . . . . . . . . . . . 6

$2.3 \mathrm{CP}$ in the Standard Model and Beyond . . . . . . . . . . . . . . . 7

2.3.1 Standard Model CKM Matrix . . . . . . . . . . . . . . . . 11

2.3.2 A Brief History of $C P$ Violation . . . . . . . . . . . . . . . . . 13

2.3.3 The Neutral K Meson . . . . . . . . . . . . . . . . . . . . . . . 13

2.3.4 The B Meson ...................... 15

2.4 Penguin Decays . . . . . . . . . . . . . . . . . . 16

$2.5 A_{C P}$ in $B \rightarrow X_{S} \gamma$ Decay ...................... 18

3 The BABAR Experiment $\quad 21$

3.1 PEP-II . . . . . . . . . . . . . . . . . . . . . 21

3.2 The BABAR Detector . . . . . . . . . . . . . . . . . . . . . . 21

3.2.1 Silicon Vertex Detector $(\mathrm{SVT}) \ldots \ldots \ldots . \ldots . \ldots . \ldots 23$

3.2 .2 Drift Chamber $(\mathrm{DCH}) \ldots \ldots \ldots \ldots$

3.2.3 Detector of Internally Reflected Cherenkov Light (DIRC) . . . . . . . 26

3.2.4 Electromagnetic Calorimeter (EMC) . . . . . . . . . . . . . 27

3.2 .5 Particle Identification . . . . . . . . . . . . . . . . . 28

3.2.6 Instrumented Flux Return (IFR) . . . . . . . . . . . . . . . 29

4 Monte Carlo and Data Samples $\quad 31$ 
5 Analysis Strategy $\quad 35$

6 Selection $\quad 41$

6.1 Initial Reconstruction . . . . . . . . . . . . . . . . . . . . . . 42

6.2 Particle Quality Criteria . . . . . . . . . . . . . . . . 43

6.2.1 High Energy $\gamma$ Selection . . . . . . . . . . . . . . . . . 44

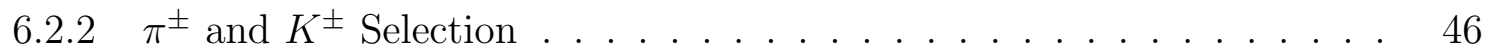

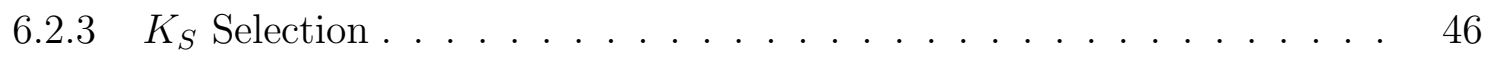

$6.2 .4 \pi^{0}$ Selection . . . . . . . . . . . . . . . . . 47

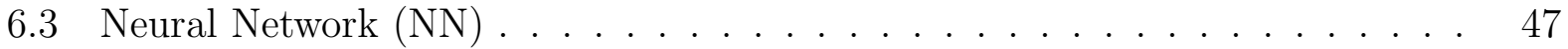

6.3.1 Input Variables for the Neural Net . . . . . . . . . . . . . . . . . . 48

6.3.2 Neural Network Basics ................... 49

6.3.3 Training the Neural Network . . . . . . . . . . . . . . 51

6.3.4 Note on Neural Networks . . . . . . . . . . . . . . . 53

6.3.5 Training/Optimization of the Neural Net . . . . . . . . . . 53

6.3.6 Comparison to a Linear Discriminant . . . . . . . . . . . . . . 54

6.4 Final Selection Cuts . . . . . . . . . . . . . . . . . . . . 55

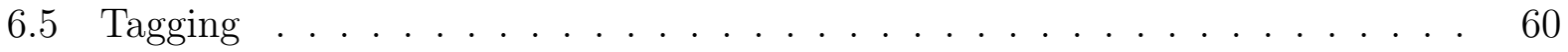

6.6 Multiple Candidates Choice . . . . . . . . . . . . . . . . . 60

$6.7 X_{S}$ Mass Cut-Off . . . . . . . . . . . . . . . . . . 61

6.8 Final Efficiencies . . . . . . . . . . . . . . . . . . 61

7 Fitting of Monte Carlo and Data $\quad 63$

7.1 Fitting of the Different Monte Carlo Categories . . . . . . . . . . . . . . 64

7.1 .1 Signal Fits . . . . . . . . . . . . . . . . 65

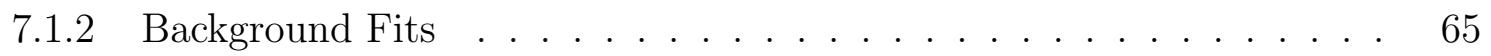


7.1 .3 Full Fits . . . . . . . . . . . . . . . . . . 68

7.2 Results from Monte Carlo Events . . . . . . . . . . . . . . . . . . . 69

$8 \mathrm{BF}\left(B \rightarrow K^{*} \gamma\right)$ Cross Check $\quad 71$

8.1 Selection Differences . . . . . . . . . . . . . . . . . 71

8.2 Fitting the $K^{*} \ldots \ldots \ldots \ldots \ldots$

$8.3 K^{*}$ Branching Fractions . . . . . . . . . . . . . . . 75

9 Systematic Uncertainties $\quad 76$

9.1 CP Asymmetry in Signal Events Due to Efficiency Biases . . . . . . . . . . . 76

10 Results $\quad 82$

$10.1 A_{C P}(b \rightarrow s \gamma)$ Numerical Inputs from Monte Carlo . . . . . . . . . . . . . 82

10.2 Expected $A_{C P}(b \rightarrow s \gamma)$ in Monte Carlo Events . . . . . . . . . . . . 83

10.3 Data Fits . . . . . . . . . . . . . . . . . . 83

10.4 Measured $A_{C P}(b \rightarrow s \gamma)$ in Data Events . . . . . . . . . . . . 85

11 Summary and Conclusions $\quad 92$

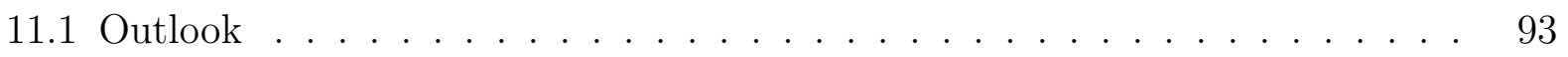

A Truth-Match for Signal and Cross-Feed $\quad 94$

$\begin{array}{lll}\text { B } & \text { The PDFs used in the fits } & 97\end{array}$

B.1 Argus Background Distribution _. . . . . . . . . . . . . . . 97

B.2 Crystal Ball Line Shape Distribution . . . . . . . . . . . . . . . . 97

$\begin{array}{lr}\text { C Toy Study } & 98\end{array}$

$\begin{array}{ll}\text { D Supplementary Systematics Section } & 100\end{array}$ 
D.1 CP Asymmetry in Mistagged Signal Monte Carlo Events due to Mis-ID Biases 100

D.2 CP Asymmetry in Cross-Feed Monte Carlo Events Due to Detector Effects . 103

D.3 Background Asymmetry of Monte Carlo Events . . . . . . . . . . . . . 104

D.4 Variation of Fitting Parameters . . . . . . . . . . . . . . . . . . 104

D.5 NN Validation . . . . . . . . . . . . . . . . . . . . . 106

D.6 Fragmentation Corrections . . . . . . . . . . . . . . . . 106

E Nuclear Cross-sections for Charged Kaons 115 


\section{List of Figures}

1 Higgs Potential for positive $\mu^{2}$. The rolling ball shows a choice of direction, thus a breaking of the symmetry of the system in favor of a ground state. . . 4

2 The running coupling constants with (right) and without (left) SUSY. . . . 5

3 One of the unitarity triangles for the B-meson. The right figure has been rotated and the sides normalized by $V_{c d} V_{c b}^{*} \ldots \ldots \ldots \ldots$. . . . . . . . . 12

4 Example Feynman diagrams for a Penguin $b \rightarrow s \gamma$ decay (left) and a tree level $b \rightarrow c$ decay (right). . . . . . . . . . . . . . . . 17

5 Schematic of SLAC, PEP-II, and placement of the BABAR detector. . . . . . 22

$6 \quad$ BABAR detecor longitudinal view. . . . . . . . . . . . . . . . 23

7 Longitudinal schematic view of the SVT. . . . . . . . . . . . . . . 24

8 Longitudinal schematic view of the DCH. The chamber is offset by 0.37 meters from the interaction point (in the forward direction). . . . . . . . . . . 25

9 Plot of $d E / d x$ in the $\mathrm{DCH}$ as a function of track momentum for different particle types. . . . . . . . . . . . . . . . . . . . 25

10 Schematic view of the DIRC . . . . . . . . . . . . . . . 26

11 Invariant $\mathrm{K} \pi$ inclusive mass spectrum with and without the use of the DIRC for kaon identification. The mass peak corresponds to the decay of the $D^{0}$

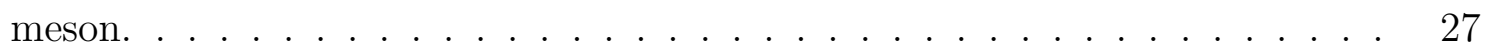

12 Schematic view of the EMC . . . . . . . . . . . . . . . . 28

13 Angular(left) and energy(right) resolution of the EMC for photons coming from neutral pions. . . . . . . . . . . . . . . . . . . . 29

14 Longitudinal schematic view of the the IFR RPC's. . . . . . . . . . . . . . 30

15 The $\left|\cos \theta_{T^{*}}\right|$ distribution. The histograms are all normalized to an area of 1.44 
16 MC mass fits to truth matched signal and flight distributions for $K_{S}$ signal events, continuum, and generic $B \bar{B}$ background. The histograms are all normalized to an area of 1 , so their shapes can be compared but not their relative heights. $K_{S}$ flight distance plot normalized to one. . . . . . . . . . . . . . 47

17 MC signal mass fits for truth matched $\pi^{0}$ s. . . . . . . . . . . . . . . . . 48

18 Shape variables going into the neural net: $\mid \cos ($ thrust $),\left|\cos \theta_{B^{*}}\right|,\left|\cos \theta^{\prime}\right|$, $W 2, R 2$ and $R 2^{\prime}$. The histograms are all normalized to an area of $1 . . . . \quad 50$

19 Basic structure of a single hidden layer neural network. Input nodes (red) corresponding to event variables are passed to "hidden" nodes (blue) as linear combinations. The line combination is then transformed bay an "activation function," in this case the $\tanh (x)$. Linear combinations of the hidden node outputs are then passed to the output node (black), where it is transformed once again by the activation function to give the final neural network output.

20 Neural net output for various MC samples: 1 is signal like, while 0 is continuum background like. The histograms are all normalized to an area of

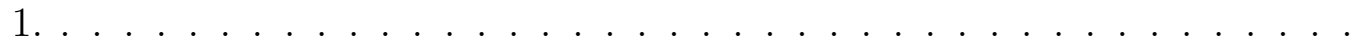

21 Plot of $\Delta E^{*}$ for signal and background. The falling off of the background $\Delta E^{*}$ distributions come from cuts made before this plot was made. The histograms are all normalized to an area of $1 \ldots \ldots \ldots \ldots$

22 Kaon momentum for $2 / 3 / 4$ body and all decays in our on-resonance sidebands. Notice the step like distribution before $0.7 \mathrm{GeV}$ due to no DIRC requirement

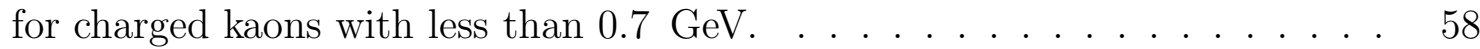

23 Charged(left) and neutral(right) bump distance distribution for signal and background MC events. The histograms are all normalized to an area of 1 . 
24 Closest masses per event (in case of overlap) to the $\pi^{0}$ (left) or $\eta$ (right) PDG masses for $X_{s} \gamma$ signal MC and generic $B \bar{B}$ background MC. The histograms are all normalized to the luminosity. . . . . . . . . . . . . . . . . . . . 59

25 Plots of continuum Monte Carlo(left) and off-resonance data(right) for the 1.5-1.9 GeV region. Both plots have be normalized to expected luminosity. .

26 Individual Monte Carlo $m_{\mathrm{ES}}$ plots for 1.1-1.5 GeV mass bin for $\bar{b}$ events: top left signal, top right cross-feed, bottom left $B \bar{B}$, and bottom right continuum. 66

27 Individual Monte Carlo $m_{\mathrm{ES}}$ plots for $b$ events in the 1.1-1.5 GeV mass bin: top left signal, top right cross-feed, bottom left $B \bar{B}$, and bottom right continuum. 67

28 Peaking Monte Carlo (Xfeed $+B \bar{B}$ ) background untagged $m_{\mathrm{ES}}$ plot for 1.1-1.5 $\mathrm{GeV}$ mass for $\bar{b}$ events. . . . . . . . . . . . . . . . . . . . . 68

29 Cross-feed, $B \bar{B}$, and continuum combined fit(left), and full Monte Carlo fit (right), for the 1.1-1.5 GeV mass bin for $\bar{b}$ events. . . . . . . . . . . 68

$30 \Delta E^{*},\left|\cos \theta_{B^{*}}\right|$, and neural net output distributions for the $K^{*}$ region. . . . 72

31 Individual Monte Carlo $m_{\mathrm{ES}}$ plots for all four $K^{*}$ modes combined: top left signal, top right Xfeed, bottom left $B \bar{B}$, and bottom right continuum. . . . . 73

32 Full signal + background fits for the combined $K^{*}$ modes; left Monte Carlo, right data . . . . . . . . . . . . . . . . . . . . . . 74

33 Overlay of Monte Carlo(triangles) and data(circles) for $\Delta E^{*}$. All cuts except our $\Delta E^{*}$ cut have been applied and $m_{\mathrm{ES}}$ asked to be greater than $5.271 \mathrm{GeV}$.

$34 m_{\mathrm{ES}}$ plot for all for data events $\left(M_{X s}=0.6-2.3 \mathrm{GeV}\right)$ : the first row shows the lepton-tagged events, the second row shows the lepton-untagged events. The left column shows the $b$ flavor sample, the left column shows the $\bar{b}$ flavor sample. 87 
35 Measured $A_{C P}$ values for all the studied categories for lepton-tagged (triangles) and untagged (circles) events. The $A_{C P}$ numbers are for the untagged

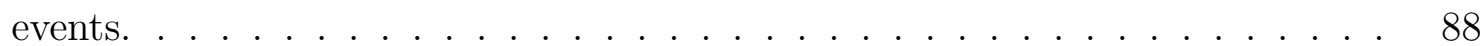

36 Measured direct $C P$ asymmetry as a function of decay mode. . . . . . . . . . 90

37 Measured direct $C P$ asymmetry as a function of hadronic mass. . . . . . . . 91

38 (Reco $M_{X s^{-}}$True $\left.M_{X s}\right)(\mathrm{GeV})$ for fully truth-matched signal events. . . . . . 95

39 (Reco $M_{X s^{-}}$True $\left.M_{X s}\right)(\mathrm{GeV})$ for events which are not "fully truth-matched," but whose tracks have an associated GHit track and the $B$ reconstructed final state is the same than the generated one. The left plots shows the events for $M_{X s}>1.1$ and the right one for $M_{X s}<1.1 \ldots \ldots \ldots$

40 (Reco $M_{X s^{-}}$True $\left.M_{X s}\right)(\mathrm{GeV})$ for Xfeed events: events whose tracks have all a GHit track associated but the reconstructed final state is different from the generated one, and all the events in which at least a $B$ daughter track does not have an associated GHit track. . . . . . . . . . . . . . . .

41 The left plot is an overlay of $9.6 \mathrm{fb}^{-1}$ of off-resonance data on top of an equal amount of $u d s$ and $c \bar{c}$ Monte Carlo. The right plot is of the off-resonance NN output (after all cuts and $\left|\Delta E^{*}\right|$ best candidate selection) for the $\bar{b}$ (circles) and $b$ (triangles) final states. . . . . . . . . . . . . . . . . . 107

42 The plots (left untagged background subtracted, right tagged) are an overlay of scaled $B \rightarrow K^{*} \gamma$ Monte Carlo signal, and on resonance data after all the selection cuts are applied. Moreover, the $m_{\mathrm{ES}}$ cut has been tightened to $5.271 \mathrm{GeV}$ to show the NN performance on "signal" like events. . . . . . . . 107

43 Total and elastic collision cross-section for $K^{+} p, K^{+} d$ (total only) and $K^{+} n$ as a function of the laboratory beam momentum and total center-of-mass energy. . . . . . . . . . . . . . . . . . . 116 
44 Total and elastic collision cross-section for $K^{-} p, K^{-} d$ (total only) and $K^{-} n$ as a function of the laboratory beam momentum and total centre-of-mass energy. . . . . . . . . . . . . . . . . . . . . . 117 


\section{List of Tables}

1 The Standard Model particles and force carriers. For the quarks and leptons the physical properties $[2,3]$ are for matter. The antimatter counterparts have opposite charge. The quarks and leptons have been split up by their generation, going from lightest to heaviest. . . . . . . . . . . . . . 2

2 Production cross-section at $\Upsilon(4 \mathrm{~S})$ resonance for BABAR . . . . . . . . . . . . 22

3 Advantages and disadvantages for the different ways to measure $A_{C P}$. . . 35

4 Studied $X_{S}$ final states and their rates (in percent) according to the Kagan and Neubert signal model [28] for two different values of the effective $b$ quark mass, $m_{b}$. The dependence of the fractions on the $b$ quark mass is a result of the different hadronic mass distributions. Note that $K_{S}$ includes the decay mode $K_{S} \rightarrow \pi^{0} \pi^{0}$, and that no constraint is made on $E_{\gamma} \ldots \ldots$

5 Reconstructed decay modes, corresponding coding referred to by "iType", and distinction among $\bar{b}$ and $b$ final states. The last is important for our definition of the $A_{C P}$. The mixed-flavor states are only used for systematic studies. . . 40

6 Cuts applied by the pion selection. "DOCA" is the distance of closest approach to the beam spot. . . . . . . . . . . . . . . . . 43

$7 \quad$ Efficiencies obtained from MC signal and background events after all initial

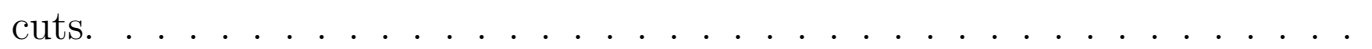

8 Average number of candidate multiplicities obtained from MC signal and background events after all initial cuts. At least one candidate is required. . . . . 44

9 For a neural round-robin training set, the optimum number of cycles to train per sub-sample, and the best neural network cut point. . . . . . . . . . . . 
10 Minimum $\pi$ and $K$ momentum cut per number of $\pi^{\prime} s$ in tag or untagged state with $1.1<M_{X s}<2.3 \mathrm{GeV} \ldots \ldots \ldots$. . . . . . . . . . . . 57

11 Average candidate multiplicities obtained from MC after all cuts are made. . 60

12 Number of signal events per $100 \mathrm{MeV}$ mass bin in $X_{S}$ (full $m_{\mathrm{ES}}$ range). The ratio of increased number of signal events to the statistical error in the peaking component of a fit to the signal background events is our criteria to choose

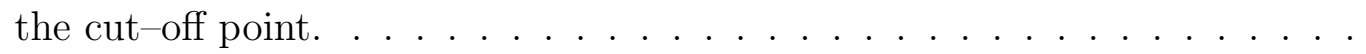

13 Final efficiencies and expected number of events for different types of signal and background definitions. Expected number of events for full $m_{\mathrm{ES}}$ distribution. 62

14 Efficiency of the cuts normalized to all cuts before (above) them for the $M_{X s}=1.1-2.3 \mathrm{GeV}$ mass range. . . . . . . . . . . . . . . . . . .

15 Functions and free parameters used to fit the various data samples. Legend: $\mathrm{A}=$ Argus function, $\mathrm{CB}=$ Crystal Ball function. . . . . . . . . . . . .

16 Values and error weighted average of truth-matched Crystal Ball parameters (both $b$ and $\bar{b}$ events fit together). See Appendix B for the Crystal Ball definition and the description of the free parameters. . . . . . . . . . . 66

17 Estimated peaking background (cross-feed and $B \bar{B}$ ) from Monte Carlo samples, expected peaking component from the Monte Carlo full fit, and actual number of Monte Carlo truth-matched signal events in the full fit for our nine data categories. The "MC Signal" plus the "Peaking Background" bins equal "Peaking Signal + Background" bins within error. The $b$ and $\bar{b}$ events have been averaged, as this is only to test that the fitting method works. . . . . .

18 Monte Carlo peaking background and peaking signal + background events as a function of the hadronic mass bins for lepton-tagged and untagged events. The quoted errors are just statistical errors. . . . . . . . . . . . . . 
19 Chosen decay mode versus true decay mode for all $K^{*}$ candidates (in percent). 72

20 Final branching fractions and statistical errors for the four reconstructed $K^{*}$ modes for the current analysis. . . . . . . . . . . . . . . . . . . 75

21 Fitted number of events in Argus and peaking the Crystal Ball fitted distributions in on-resonance sideband data, on-resonance continuum enriched and suppressed data, antiveto sample and off-resonance data for $b$ and $\bar{b}$ flavors. The corresponding $C P$ asymmetry is computed. . . . . . . . . . . . . 79

22 CP asymmetry dependence on the charged kaon momentum in on-resonance sideband data. . . . . . . . . . . . . . . . . . . . . . . 80

$23 C P$ asymmetry dependence on the $K_{S}$ momentum in on-resonance sideband data. . . . . . . . . . . . . . . . . . . . . . . . . . .

24 Shifts due to the fake detector $C P$ asymmetry in reconstructed data signal events as a function of the hadronic mass bins for lepton-tagged $\left(\begin{array}{c}\text { Shift } \\ A_{\text {CPag. }}\end{array}\right)$ and untagged events $\left(A_{C P \text { Shtag. }}^{\text {Shift }}\right)$. This is our only systematic error. . . . . . .

25 Monte Carlo peaking background, full Monte Carlo peaking events and reconstructed number of events from the difference of the full Monte Carlo peaking events and Monte Carlo peaking background as a function of the hadronic mass bins for lepton-tagged and untagged events. The quoted errors are just

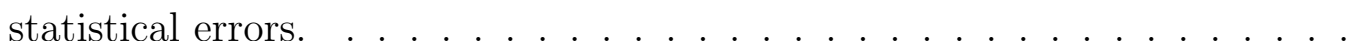

26 The dilution difference, $\Delta D$, and average, $\langle D\rangle$, and mis-tag rates $(\omega$ and $\bar{\omega}$ for $b$ and $\bar{b}$ events, respectively) as derived from signal Monte Carlo events in Section D.1 for lepton-tagged and untagged events. Where there is - means that there were not enough statistics. . . . . . . . . . . . . 
27 Shifts due to the fake detector $C P$ asymmetry in reconstructed data signal events as a function of the hadronic mass bins for lepton-tagged $\left(A_{C P}\right.$ Shift

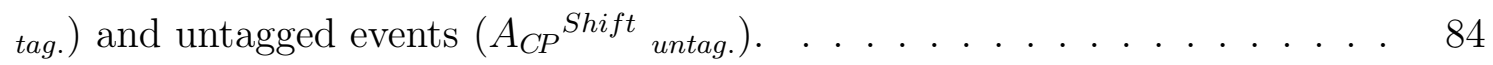

28 Expected $A_{C P}$ (in Monte Carlo) as a function of the hadronic mass bins. The $A_{C P}$ is not corrected by the mistag rate and the detector shift. The quoted errors are just statistical errors. . . . . . . . . . . . . . . . .

29 Data peaking yield and corresponding reconstructed number of events (after the Monte Carlo peaking background from Table 25 is subtracted) as a function of the hadronic mass bins. The quoted errors are just statistical errors. 86

30 Measured final $A_{C P}$ in data as a function of the hadronic mass bins. . . . . . 86

$31 C P$ asymmetries in various models. Ranges are approximate using current knowledge of $B \rightarrow X_{s} \gamma$ decays. . . . . . . . . . . . . . . . . . . . 93

32 This table shows results from toy data set fits to the peaking components of Monte Carlo samples, number of expected peaking background from crossfeed and $B \bar{B}$ Monte Carlo, their combined fit, fit after adding the continuum Monte Carlo, and then fit after adding the signal Monte Carlo. One can subtract the "Peaking Combined Xfeed $+B \bar{B}$ " from the "Peaking Full Fit" column to get the result in the "Signal" column. These numbers come from the toy study data set, and are to be used to show that the fitting technique is consistent. . . . . . . . . . . . . . . . . . . . 
33 The pull error and statistical error for all the toy examples. Large statistical errors come from low statistics, e.g. for the $1.6-1.8 \mathrm{GeV}$ bin, the $783 \%$ statistical error comes from an expected peaking component of $1.2 \pm 9.4$ events. These numbers come from the toy study data set, and are to be used to show that the fitting technique is consistent. All possible pulls correspond to pulls of $<1$ event in the final fit, and are not consistently in the positive or negative yield direction. . . . . . . . . . . . . . . . . . .

34 Cases in which mistagged signal events generate the same or the opposite flavor due to charge confusion or pion mis-ID as a Kaon. It is assumed that the signal events are not re-classified as cross-feed as a result of the mis-

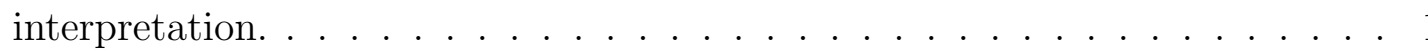

35 The mistag rate for signal events lepton-tagged $\left(\omega_{\text {tag }}\right)$ and untagged $\left(\omega_{\text {untag }}\right)$ events is shown for the $b$ and $\bar{b}$ flavors. Monte Carlo events are re-weighted. Where there is a "-," means that there were not enough statistics. . . . . . .

36 Fake $A_{C P}$ in mistagged events due to detector bias as a function of the hadronic mass bins. The events are split into lepton-tagged $\left(A_{C P}{ }_{\text {tag. }}^{\text {fake }}\right)$ and untagged events $\left(A_{C P}^{\text {fake }}{ }_{\text {untag. }}\right)$. Monte Carlo events are re-weighted. Where there is a "-," means that there were not enough statistics. . . . . . . . . . . . . . . 102

37 Fit peaking component for $b$ and $\bar{b}$ cross-feed Monte Carlo events and corresponding $A_{C P}$ for lepton-tagged and untagged events. The events are scaled to luminosity. . . . . . . . . . . . . . . . . . . . .

38 Fitted number of events in Argus and peaking the Crystal Ball fitted distributions in generic $B \bar{B}$ and continuum Monte Carlo events for $b$ and $\bar{b}$ flavors. The corresponding $C P$ asymmetry is computed. The events are leptonuntagged. 
39 Number of peaking background events and number of peaking signal and background events, for lepton-tagged and untagged events, with $K^{ \pm}$yield increased by 10\%. . . . . . . . . . . . . . . . . . 108

40 Kaon efficiency $\varepsilon\left(K^{+}\right)$and $\varepsilon\left(K^{-}\right)$for positively and negatively charged kaons during RUN1 and RUN2 as a function of the kaon momentum and the resulting detector efficiency $C P$ asymmetry. The kaon efficiency is obtained from the official PID tables. The apparent $C P$ asymmetry is defined as $A_{C P}{ }^{D e t}=$ $\frac{\varepsilon\left(K^{-}\right)-\varepsilon\left(K^{+}\right)}{\varepsilon\left(K^{-}\right)+\varepsilon\left(K^{+}\right)}$. . . . . . . . . . . . . . . . . . . .

41 Apparent $C P$ asymmetry in reconstructed Monte Carlo signal events due to the detector efficiency bias as a function of the hadronic mass bins for leptontagged $\left(A_{C P}^{\text {Det }}{ }_{\text {tag. }}\right)$ and untagged events $\left(A_{C P}^{\text {Det }}{ }_{\text {untag. }}\right) . \ldots \ldots$. . . . . . 110

42 Apparent $C P$ asymmetry in reconstructed Monte Carlo signal events due to the detector efficiency bias as a function of the hadronic mass bins for leptontagged $\left(A_{C P}{ }^{\text {Det }}{ }_{\text {tag. }}\right)$ and untagged events $\left(A_{C P}{ }^{\text {Det }}{ }_{\text {untag. }}\right)$. The signal events are re-weighted according to the $A_{C P}$ found in data events. . . . . . . . . . . 110

43 Pion to Kaon mis-ID rates $\varepsilon\left(\pi^{+} \rightarrow K^{+}\right)$and $\varepsilon\left(\pi^{-} \rightarrow K^{-}\right)$for positively and negatively charged pions during RUN1 and RUN2 as a function of the pion momentum and the resulting fake $C P$ asymmetry. The pion mis-ID rates are obtained from the official PID tables. The fake $C P$ asymmetry is defined as $A_{C P}$ fake $=\frac{\varepsilon\left(\pi^{-} \rightarrow K^{-}\right)-\varepsilon\left(\pi^{+} \rightarrow K^{+}\right)}{\varepsilon\left(K^{-}\right)+\varepsilon\left(K^{+}\right)} \ldots \ldots \ldots \ldots \ldots$

44 Cross-feed events that can contribute to $C P$ asymmetries due to replacement of a final state particle. The table also includes cases where not mixed-flavors change to mixed-flavors, and vice-versa. . . . . . . . . . . . . . . . 112 
45 Cross-feed events that can contribute to $C P$ asymmetries due to the addition or removal of a pion in the final state. Again note that there are cases where not mixed-flavors change to mixed-flavors, and vice-versa. . . . . . . . . . . 113

46 Comparison of $b$ and $\bar{b}$ Argus parameters for a fit to the lepton-untagged continuum $\mathrm{MC}$ events. . . . . . . . . . . . . . . . . . . . . 113

47 Comparison of $\bar{b}$ and $b$ floated Crystal Ball parameters for lepton-untagged signal MC events (normally fixed to a weighted average). . . . . . . . . . . . 114

48 Comparison of $b$ and $\bar{b}$ Argus parameters and corresponding yields for a fit to the lepton-untagged cross-feed and $B \bar{B}$ MC events. . . . . . . . . . . . . . 114

$49 C P$ asymmetry (last column) we find as a function of the kaon momentum (first column). The other columns show the details needed to extract the $A_{C P}$ : the second and the fourth columns show the kaon cross-section while the third and fifth columns show the percentage of lost kaons due to the nuclear interaction at $90^{\circ} \ldots \ldots \ldots \ldots \ldots \ldots$ 


\section{Abstract}

This dissertation describes a measurement of the direct $C P$ asymmetry between inclusive $b \rightarrow s \gamma$ and $\bar{b} \rightarrow \bar{s} \gamma$ decays. This asymmetry is expected to be less than 0.01 in the Standard Model, but could be enhanced up to the order of 0.10 by non-Standard Model contributions. We use a sample of 89 million $B \bar{B}$ pairs recorded with the BABAR detector at PEP-II, from which we reconstruct a set of 12 exclusive $b \rightarrow s \gamma$ final states containing one charged or neutral kaon and one to three pions. We measure an asymmetry of $A_{C P}(b \rightarrow s \gamma)=$ $0.025 \pm 0.050$ (stat. $) \pm 0.015$ (syst.), corresponding to an allowed range of $-0.06<A_{C P}(b \rightarrow$ $s \gamma)<+0.11$ at $90 \%$ confidence level. 


\section{Introduction}

Why can't ignorance be painful?

We currently live in a matter-dominated universe. But, 13.7 billion years ago when the universe was created. there were equal parts of matter and antimatter. If this 50:50 ratio of matter to antimatter remained constant throughout the lifetime of the universe we would not be here. So the question remains, "Where did all the antimatter go?"

Our best answer is that there are tiny differences in the interactions and decays of matter and antimatter. These differences were first seen in the 1960's when the decay of the Kmeson was found to be slightly different than that of its antimatter counterpart ( $\bar{K}$-meson). This difference is called $C P$ violation $(C P$ is the changing of matter into antimatter and a simultaneous mirror reflection).

Our current model of how the universe works at the most fundamental level accounts for the $C P$ violation seen in the $\mathrm{K}$-meson system. Yet the same model does not account for the matter - antimatter discrepancies we see within our universe.

To further probe this matter - antimatter asymmetry we study the B-meson. Like the Kmeson the B-meson can violate $C P$, but its heavier mass allows for decay into more possible $C P$ violating states. Even though the measured $C P$ violation of the B-meson is larger than that of the K-meson, the violating effects are still quite small. So we need a large number of B-mesons for our studies.

To get the large number of B-mesons needed, we use the PEP-II storage rings and Stanford Linear Accelerator Center (SLAC) Linac to produce almost 180 million B-mesons for this analysis. The BABAR detector then identifies these B-mesons decays, and their decay

constituents. From these reconstructed decays we extract the small number of B-mesons that could violate $C P$ for further study. 


\subsection{The Standard Model}

The Standard Model [1] is the best theory that describes (and accurately predicts) the interactions between fundamental pieces of matter. Specifically, the Standard Model is a quantum field theory that is consistent with Special Relativity, and describes the strong, weak, and electromagnetic interactions between quarks and leptons.

Quarks and leptons are the basic building blocks of matter, where the first generation (see Table 1) dominates the present universe. Second and third generation quarks and leptons are heavy unstable elementary particles that are only observed in high-energy processes.

\begin{tabular}{|l|c|c|c|}
\hline Particle & Spin & Electric Charge & Mass $(\mathrm{GeV})$ \\
\hline \hline First Generation Quarks and Leptons & & & \\
u (up) & $1 / 2$ & $2 / 3$ & $0.0015-0.0045$ \\
d (down) & $1 / 2$ & $-1 / 3$ & $0.005-0.0085$ \\
e (electron) & $1 / 2$ & -1 & $5.11 \times 10^{-4}$ \\
$\nu_{e}$ (electron neutrino) & $1 / 2$ & 0 & $<10^{-8}$ \\
\hline Second Generation Quarks and Leptons & & & \\
c (charm) & $1 / 2$ & $2 / 3$ & $1.0-1.4$ \\
s (strange) & $1 / 2$ & $-1 / 3$ & $0.080-0.155$ \\
$\mu$ (muon) & $1 / 2$ & -1 & 0.106 \\
$\nu_{\mu}$ (muon neutrino) & $1 / 2$ & 0 & $<2 \times 10^{-4}$ \\
\hline Third Generation Quarks and Leptons & & & \\
t (top) & $1 / 2$ & $2 / 3$ & $174.3 \pm 5.1$ \\
b (bottom) & $1 / 2$ & $-1 / 3$ & $4.0-4.5$ \\
$\tau$ (tau) & $1 / 2$ & -1 & 1.77 \\
$\nu_{\tau}$ (tau neutrino) & $1 / 2$ & 0 & $<0.02$ \\
\hline \hline Bosons & & & 0 \\
g (gluon) [strong] & 1 & 0 & 0 \\
$\gamma$ (photon)[EM] & 1 & 0 & 80.4 \\
$W \pm$ [weak] & 1 & \pm 1 & 91.2 \\
Z [weak] & 1 & 0 & \\
\hline
\end{tabular}

Table 1: The Standard Model particles and force carriers. For the quarks and leptons the physical properties $[2,3]$ are for matter. The antimatter counterparts have opposite charge. The quarks and leptons have been split up by their generation, going from lightest to heaviest. 
The Standard Model describes the interactions of quarks and leptons by combining the theory of electroweak interactions (a combination of electromagnetic and weak interactions) with quantum chromodynamics (strong interactions). Both electroweak and quantum chromodynamics are gauge field theories, whose forces are mediated by gauge bosons. These mediating gauge bosons are listed in Table 1, and briefly described below:

Gluon : Eight species of the gluon mediate the nuclear strong force.

Photon : The photon mediates the electromagnetic interaction. The electromagnetic force is on the order of $10^{-3}$ times the strength of the strong force.

$W^{ \pm}$and $Z$ : The $\mathrm{W}$ and $\mathrm{Z}$ bosons mediate the weak nuclear force. The weak force is on the order of $10^{-14}$ times the strength of the strong force.

Finally, all of the elementary particles in Table 1 have masses. In the Standard Model, particle mass is acquired through the Higgs mechanism. Specifically, the Standard Model Higgs is a complex scalar field with only one Higgs doublet, and a non-zero vacuum expectation value. The potential of the field is given by (see Figure 1):

$$
V=-\mu^{2}|\phi|^{2}+\lambda|\phi|^{4}
$$

where $\phi$ is the Higgs field.

Through the electroweak symmetry breaking ${ }^{1}$, the gauge bosons and Higgs receive their masses. At first order, the mass formulae for the bosons and Higgs are given by:

$$
m_{W}=\frac{1}{2} g v, \quad m_{Z}=\frac{1}{2} \sqrt{g+g^{\prime}} v,
$$

\footnotetext{
${ }^{1}$ Spontaneous (electroweak) symmetry breaking is where a system that is symmetric goes into a ground state that is not symmetric. An example of this phenomenon is a ball sitting on top of a hill as in Figure 1 . The ball starts in a symmetric non-stable state, but when the ball starts rolling, symmetry has been broken since one direction has been chosen.
} 


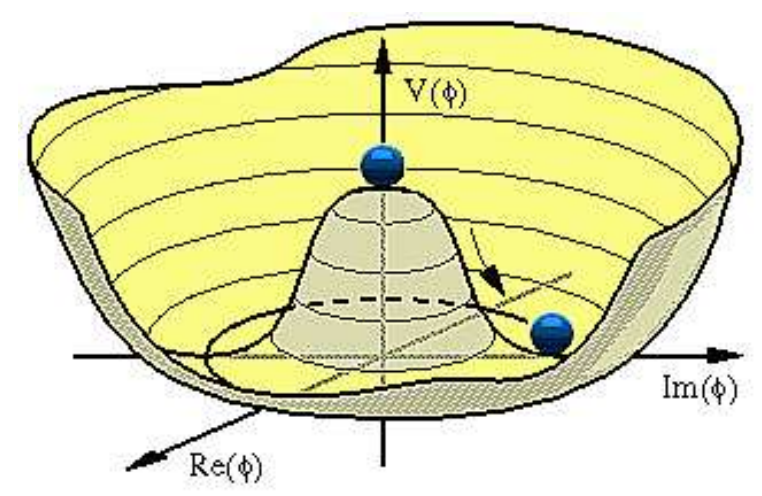

Figure 1: Higgs Potential for positive $\mu^{2}$. The rolling ball shows a choice of direction, thus a breaking of the symmetry of the system in favor of a ground state.

$$
m_{H}=\sqrt{\lambda} v
$$

Where $v(\simeq 246 \mathrm{GeV}[4])$ is the Higgs vacuum expectation value. $g$ and $g^{\prime}$ are the electroweak gauge coupling constants [5] which are related by the Weinberg (weak) angle $\theta_{W}[6]$ :

$$
\tan \theta_{W}=g^{\prime} / g, \quad \sin ^{2} \theta_{W}=1-\frac{m_{W}^{2}}{m_{Z}^{2}}=0.2221 \pm 0.0004 .
$$

The Weinberg angle is a fundamental parameter in the Standard Model that relates the relative strength of the $\mathrm{W}$ and $\mathrm{Z}$ electromagnetic couplings.

Equations 2 imply that boson mass is determined by the strength of its interaction with the Higgs field. Therefore, the measurement of the coupling constants related to the Higgs boson is an important check of the mass generation in the Standard Model. Specifically, the formula $m_{H}=\sqrt{\lambda} v$ suggest that the mass of the Higgs boson reflects the strength of electroweak symmetry breaking dynamics.

Although the Higgs boson mass is a free parameter within the Standard Model, we can determine its upper and lower bounds. The current experimental constraint on the Higgs mass is $114<M_{\text {Higgs }}<193 \mathrm{GeV}$ (at the $95 \%$ confidence limit). The lower limit on the Higgs mass comes from direct searches at the LEP experiments [7]. While the upper limit comes 
from precision electroweak measurements of the $W$ and $Z$ bosons at SLAC and CERN [8].

It is notable that even though the Standard Model accurately predicts many phenomena in sub-atomic interactions, we know that the Standard Model is incomplete. One of the major questions is, "Why are the strengths of the fundamental forces so different?" We know that the coupling constants of the strong, weak, and electromagnetic forces change with the energy at which scale they are measured. We also think that at the time of the Big Bang, all forces had the same strength. Yet when we run these coupling constants (by extrapolating current knowledge of the coupling constants to higher energies) we see that they do not unite (see Figure 2).

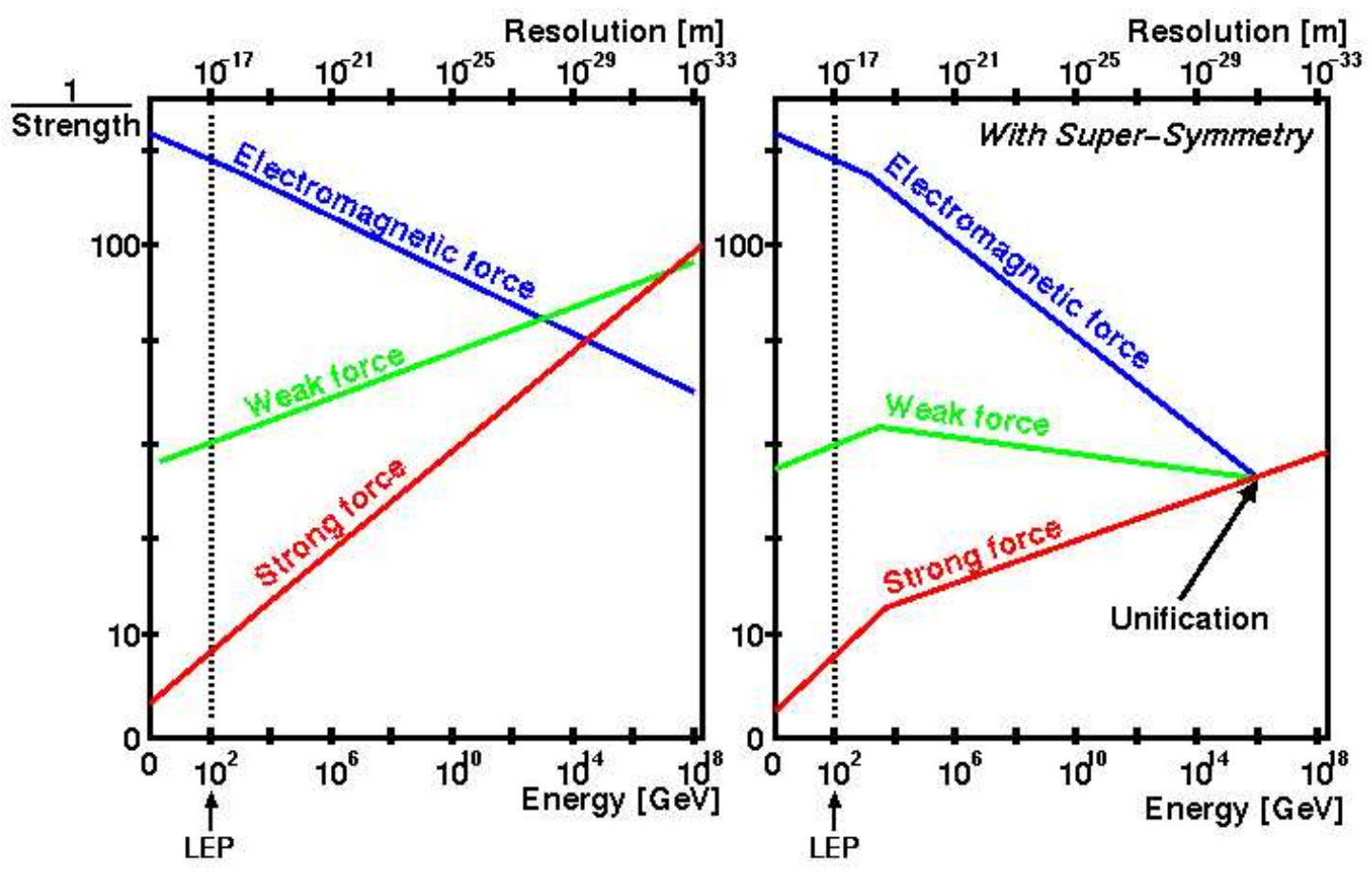

Figure 2: The running coupling constants with (right) and without (left) SUSY.

The shortcomings of the Standard Model are described as the Hierarchy Problem, and comes about from radiative corrections to mass at high energies [4]. There are a number of 
extensions to the Standard Model that solve the Hierarchy Problem. Three of these solutions are:

Technicolor : Technicolor models are GUT theories which do not have a scalar Higgs field. Instead, they have a larger number of fermion fields than the Standard Model. This larger group is spontaneously broken down to the Standard Model as fermion condensates form.

Supersymmetry : Supersymmetry is a symmetry that relates bosons and fermions. In supersymmetric theories, every fundamental fermion has a superpartner that is a boson, and vice versa.

Superstring Theory : Superstring theory is an attempt to explain all of the particles and fundamental forces of nature in one theory by modeling them as vibrations of tiny supersymmetric strings.

For the rest of the paper we will only concentrate on Supersymmetric extensions to the Standard Model.

\subsection{Supersymmetric Extensions to the Standard Model}

In SUSY, all fundamental particles have superpartners. For each fermion, there is a spin zero superpartner, and for each force-carrying boson, there is a spin 1/2 superpartner.

In SUSY there is no Hierarchy Problem. The divergence in scalar mass renormalization that is present in the Standard Model is canceled out due to the coupling of Standard Model particles with their SUSY superpartners [9]. Thus, the coupling constants unify at high energies and the Hierarchy Problem goes away (see Figure 2) [4].

The simplest supersymmetric extension (SUSY) of the Standard Model is called the Minimally Supersymmetric Standard Model (MSSM) [10]. The Higgs sector of the MSSM 
is comprised of two complex scalar fields with two Higgs doublets [10]. The Higgs doublet fields $H_{1}$ and $H_{2}$ are introduced for the down-type quark/lepton Yukawa coupling and the up-type quark Yukawa coupling respectively. In this case, there are now five Higgs bosons: a charged Higgs pair $\left(H^{ \pm}\right)$, one $C P$ odd scalar Higgs $(A)$, and two $C P$ even scalars Higgs $(h$ and $H)$.

Since MSSM Higgs self-interactions are not independent parameters, they can all be expressed in terms of electroweak gauge coupling constants. Therefore, all MSSM Higgs sector parameters at tree level are determined by two free parameters, $\tan \beta\left(\tan \beta \equiv\left\langle H_{2}\right\rangle /\left\langle H_{1}\right\rangle\right)$ and $m_{A}$ (mass of the $C P$ odd SUSY Higgs). So for $m_{A} \gg m_{Z}\left(m_{Z}\right.$ is the mass of the Standard Model Z boson), the expressions for the Higgs masses are:

$$
m_{h}^{2} \simeq m_{Z}^{2} \cos ^{2} 2 \beta, \quad m_{H}^{2} \simeq m_{A}^{2} \sin ^{2} 2 \beta, \quad m_{H^{ \pm}}^{2}=m_{A}^{2}+m_{W}^{2}
$$

where $m_{W}$ is the mass of the Standard Model W boson.

\subsection{CP in the Standard Model and Beyond}

In the Standard Model $C P$ eigenstates of mesons may not be the same as their weak interaction eigenstates, and a framework is needed which quantifies the relationship between them. We begin by defining the Standard Model operators charge $(C)$, parity $(P)$, and time $(T)$ as:

Charge : The charge conjugation operator transforms the particle to its anti-particle without changing position or momentum.

Parity : The parity operator reflects the spatial coordinates through the origin. This is like a mirror image followed by a 180-degree rotation.

Time : The time reversal operator leaves the spatial direction unchanged, but reverses the momentum and angular momentum. 
We note that theory states that $C P T$ is conserved in interactions [11], and current experimental results are consistent with the conservation of $C P T$ [12]. This means that the mass, charge, and lifetime are the same for matter and antimatter, but decay rates can be different. Thus, $C P$ or $\mathrm{T}$ can be violated in the Standard Model.

Next we put forth the three forms of $C P$ violation in the Standard Model that arise from weak eigenstates and mass eigenstates not being the same: direct $C P$ violation, $C P$ violation in mixing, and $C P$ violation in the interference between decays:

Direct $C P$ violation comes from $C P$ violation in decay, where a decay process and its $C P$ conjugate process have different phases.

$C P$ violation in mixing appears when particle mass eigenstates differ from the $C P$ eigenstates.

$C P$ violation in interference occurs when final $C P$ states are accessible from different initial $C P$ states without mixing.

We can now approach the three forms of $C P$ violation in a generalized field theory. We start with $C P$ violation in mixing by defining a weakly decaying neutral meson $X^{0}$ as an arbitrary linear combination of the flavor eigenstates. If we assume that the $X^{0}$ mixes with its $C P$ conjugate according to the time dependent Schrodinger equation we have:

$$
\begin{gathered}
\alpha\left|X^{0}\right\rangle+\beta\left|\bar{X}^{0}\right\rangle \\
i \frac{\partial}{\partial t}\left(\begin{array}{c}
\alpha \\
\beta
\end{array}\right)=\mathcal{H}\left(\begin{array}{c}
\alpha \\
\beta
\end{array}\right) \equiv\left(\begin{array}{cc}
m_{11}-\frac{1}{2} i \Gamma_{11} & m_{12}-\frac{1}{2} i \Gamma_{12} \\
m_{21}-\frac{1}{2} i \Gamma_{21} & m_{22}-\frac{1}{2} i \Gamma_{22}
\end{array}\right)\left(\begin{array}{c}
\alpha \\
\beta
\end{array}\right) .
\end{gathered}
$$

The $m$ and $\Gamma$ terms represent the mixing and decay features of the state. The off-diagonal elements of the matrix are potentially complex. Specifically, the phase of the off-diagonal elements corresponds to the phase of the mixing, and $\Gamma_{12}$ represents the coupling to common 
decay modes of the $X^{0}$ and $\bar{X}^{0}\left(C P T\right.$ invariance guarantees that $m_{11}=m_{22} \equiv m, \Gamma_{11}=$ $\Gamma_{22} \equiv \Gamma, m_{21}=m_{12}^{*}$, and $\left.\Gamma_{21}=\Gamma_{12}^{*}.\right)$. Equation 5 then becomes:

$$
i \frac{\partial}{\partial t}\left(\begin{array}{c}
\alpha \\
\beta
\end{array}\right)=\left(\begin{array}{cc}
m-\frac{1}{2} i \Gamma & m_{12}-\frac{1}{2} i \Gamma_{12} \\
m_{12}^{*}-\frac{1}{2} i \Gamma_{12}^{*} & m-\frac{1}{2} i \Gamma
\end{array}\right)\left(\begin{array}{c}
\alpha \\
\beta
\end{array}\right),
$$

and the mass eigenstates are simply the eigenvectors of the Hamiltonian:

$$
\begin{gathered}
\left|X_{H}\right\rangle=p\left|X^{0}\right\rangle-q\left|\bar{X}^{0}\right\rangle, \\
\left|X_{L}\right\rangle=p\left|X^{0}\right\rangle+q\left|\bar{X}^{0}\right\rangle,
\end{gathered}
$$

where:

$$
\begin{gathered}
|p|^{2}+|q|^{2}=1, \\
\frac{q}{p}=-\frac{2\left(m_{12}^{*}-\frac{i}{2} \Gamma_{12}^{*}\right)}{\Delta m-\frac{i}{2} \Delta \Gamma} .
\end{gathered}
$$

The mass difference and decay width difference between $\left|X_{H}\right\rangle\left(M_{H}, \Gamma_{H}\right)$ and $\left|X_{L}\right\rangle\left(M_{L}\right.$, $\left.\Gamma_{L}\right)$ can be obtained by diagonalizing the mixing matrix:

$$
\alpha=\left|m_{12}\right|^{2}-\frac{1}{4}\left|\Gamma_{12}\right|^{2}, \quad \beta=\mathcal{R} e\left(m_{12} \Gamma_{12}^{*}\right),
$$

then:

$$
\begin{gathered}
\Delta m=\left|M_{H}-M_{L}\right|=\sqrt{2 \alpha-2 \sqrt{\alpha^{2}-\beta^{2}}}, \\
\Delta \Gamma=\left|\Gamma_{H}-\Gamma_{L}\right|=4 \beta / \Delta m .
\end{gathered}
$$

We see that an initially pure $\left|X^{0}\right\rangle$ state will time evolve as a superposition of the mass eigenstates $\left|X_{L}\right\rangle$ and $\left|X_{H}\right\rangle$. The time evolution can be fully expressed in terms of the physically intuitive quantities $m, \Gamma, \Delta m$, and $\Delta \Gamma$.

$$
\begin{aligned}
\left|X^{0}(t)\right\rangle & =g_{+}(t)\left|X^{0}\right\rangle+(q / p) g_{-}(t)\left|\bar{X}^{0}\right\rangle, \\
\left|\bar{X}^{0}(t)\right\rangle & =(p / q) g_{-}(t)\left|X^{0}\right\rangle+g_{+}(t)\left|\bar{X}^{0}\right\rangle,
\end{aligned}
$$


where

$$
\begin{aligned}
& g_{+}(t)=e^{-i m t} e^{-\Gamma t / 2}\left(e^{-i \Delta m t / 2} e^{-\Delta \Gamma t / 2}+e^{+i \Delta m t / 2} e^{+\Delta \Gamma t / 2}\right), \\
& g_{-}(t)=e^{-i m t} e^{-\Gamma t / 2}\left(e^{-i \Delta m t / 2} e^{-\Delta \Gamma t / 2}-e^{+i \Delta m t / 2} e^{+\Delta \Gamma t / 2}\right) .
\end{aligned}
$$

Now we can move on to $C P$ violation in decay. We consider the decay amplitudes for $C P$ conjugate states $X$ and $\bar{X}$ to decay into final states $f$ and $\bar{f}$ respectively. If we call these amplitudes $A_{f}$ and $\bar{A}_{\bar{f}}$, the quantity $\left|\frac{\bar{A}_{\bar{f}}}{A_{f}}\right|$ is phase-convention independent.

There are two types of phases present in the amplitudes $A_{f}$ and $\bar{A}_{\bar{f}}$ :

1. Those arising from complex parameters in the Lagrangian: In the Standard Model, such phases enter only via the electroweak CKM mass mixing matrix (see Section 2.3.1), and are often called "weak" phases. Such phases appear in $A_{f}$ and $\bar{A}_{\bar{f}}$ with opposite signs.

2. Those arising from contributions of intermediate on-shell states: Since such contributions are usually dominated by strong interactions, these are often called "strong" phases. Since they do not intrinsically violate $C P$, such phases appear in $A_{f}$ and $\bar{A}_{\bar{f}}$ with like signs.

If we separate each decay channel's contribution to the amplitude into magnitude $A_{i}$, "weak-phase" term $e^{i \phi_{i}}$ and "strong-phase" term $e^{i \delta_{i}}$, we obtain:

$$
\left|\frac{\bar{A}_{\bar{f}}}{A_{f}}\right|=\left|\frac{\sum_{i} A_{i} e^{i \delta_{i}-\phi_{i}}}{\sum_{i} A_{i} e^{i \delta_{i}+\phi_{i}}}\right| .
$$

From this we can set that "direct" $C P$ violation only occurs if two terms with differing weak phases acquire different strong phases:

$$
|A|^{2}-|\bar{A}|^{2}=-2 \sum_{i, j} A_{i} A_{j} \sin \left(\phi_{i}-\phi_{j}\right) \sin \left(\delta_{i}-\delta_{j}\right)
$$


Furthermore, the rate asymmetry can be expressed in terms of the decay amplitudes:

$$
\alpha_{s}=\frac{1-|\bar{A} / A|^{2}}{1+|\bar{A} / A|^{2}}
$$

\subsubsection{Standard Model CKM Matrix}

Finally, we need a way of relating the weak interaction eigenstates to the mass eigenstates. We do this by choosing a reference frame where only the down type quark of each generation $(d, s, b)$ have different weak and mass eigenstates. If we define the weak interaction eigenstates as $\left(d^{\prime}, s^{\prime}, b^{\prime}\right)$, we can then generate a relationship between their weak and mass eigenstates. This relationship was first developed by Cabibbo, Kobayashi, and Maskawa, and is known as the $C K M$ matrix [13]. The matrix is then defined by:

$$
\left(\begin{array}{c}
d^{\prime} \\
s^{\prime} \\
b^{\prime}
\end{array}\right)=\left(\begin{array}{ccc}
V_{u d} & V_{u s} & V_{u b} \\
V_{c d} & V_{c s} & V_{c b} \\
V_{t d} & V_{t s} & V_{t b}
\end{array}\right)\left(\begin{array}{c}
d \\
s \\
b
\end{array}\right) \equiv V_{C K M}\left(\begin{array}{c}
d \\
s \\
b
\end{array}\right) .
$$

Where the CKM matrix elements are known to be (90\% confidence limit assuming only 6 quarks) [14]:

$$
\left(\begin{array}{ccc}
0.9741-0.9756 & 0.219-0.226 & 0.0025-0.0048 \\
0.219-0.226 & 0.9732-0.9748 & 0.038-0.044 \\
0.004-0.014 & 0.037-0.044 & 0.9990-0.9993
\end{array}\right)
$$

From probability requirements, the CKM matrix must be unitary, and from unitarity constraints, the CKM matrix can be characterized by three real variables and one complex phase, which allows for $C P$ violation within the Standard Model.

There are then multiple ways that we can transform the CKM matrix for easier interpretation. From experimental results we know that $V_{u s} \approx 0.22$ [14], and we can expand about this parameter $\lambda=\left|V_{u s}\right|$ due to its small value. This leads to $V_{c b}=\mathcal{O}\left(\lambda^{2}\right)$ and $V_{u b}=\mathcal{O}\left(\lambda^{3}\right)$. 
To have expansion coefficients of order unity, we construct the parameters: $A, \rho$ and complex phase $\eta$. This expansion is known as the Wolfenstein parameterization [15]:

$$
V_{C K M}=\left(\begin{array}{ccc}
1-\lambda^{2} / 2 & \lambda & A \lambda^{3}(\rho-i \eta) \\
-\lambda & 1-\lambda^{2} & A \lambda^{2} \\
A \lambda^{3}(1-\rho-i \eta) & -A \lambda^{2} & 1
\end{array}\right)
$$

Another way of visualizing the $C K M$ matrix is by using its unitarity constraint: $V_{n l} V_{m l}=$ 0 for $n \neq m$. We can write the CKM matrix elements as a set of three linear equations:

$$
\begin{aligned}
& V_{u d} V_{u s}^{*}+V_{c d} V_{c s}^{*}+V_{t d} V_{t s}^{*}=0, \\
& V_{u s} V_{u b}^{*}+V_{c d} V_{c b}^{*}+V_{t d} V_{t b}^{*}=0, \\
& V_{u d} V_{u b}^{*}+V_{c d} V_{c b}^{*}+V_{t d} V_{t b}^{*}=0 .
\end{aligned}
$$

Each of these relations requires the sum of the complex quantities to be zero, so we can geometrically represent these as a triangle in a complex plane. One of the "unitarity triangles" can be seen in Figure 3.
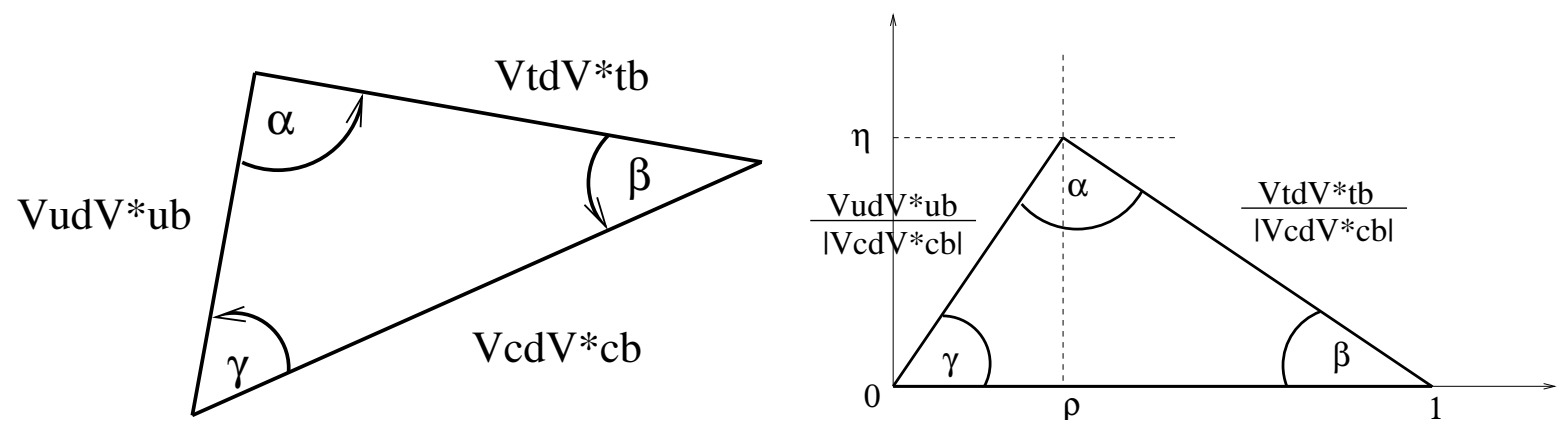

Figure 3: One of the unitarity triangles for the B-meson. The right figure has been rotated and the sides normalized by $V_{c d} V_{c b}^{*}$. 


\subsubsection{A Brief History of $C P$ Violation}

Until the 1960's CP was assumed to be an observed symmetry of nature. But, in 1964, Christensen, Cronin, Fitch, and Turlay discovered the $C P$ violating decay $K_{L} \rightarrow \pi \pi[16]$. Since then, the thrust of $C P$ violation studies in sub-atomic physics has been in the K-meson and B-meson realm. The following is a very brief introduction to $\mathrm{K}$-meson and $\mathrm{B}$-meson $C P$ physics.

\subsubsection{The Neutral K Meson}

Neutral K-mesons are mesons with quark content $\bar{K}^{0}=s \bar{d}, K^{0}=\bar{s} d$, and when K-mesons decay to $C P$ eigenstates, the lifetimes differ hugely between the mass eigenstates. Therefore, it is convenient to define "long" and "short" mass eigenstates in terms of their lifetimes [17]:

$$
\begin{gathered}
\tau_{S}=(8.927 \pm 0.009) \times 10^{-11} s \\
\tau_{L}=(5.17 \pm 0.04) \times 10^{-8} s
\end{gathered}
$$

where the subscripts $S$ and $L$ refer to the short and long-lived mass eigenstates:

$$
\begin{aligned}
& \left|K_{S}\right\rangle=p\left|K^{0}\right\rangle+q\left|\bar{K}^{0}\right\rangle, \\
& \left|K_{L}\right\rangle=p\left|K^{0}\right\rangle-q\left|\bar{K}^{0}\right\rangle,
\end{aligned}
$$

such that $\Delta \Gamma_{K}<0$ by construction. Furthermore, the K-meson mass difference has been measured to be [17]:

$$
\Delta m_{K} \equiv M_{L}-M_{S}=(3.491+-0.009) \times 10^{-15} \mathrm{GeV} .
$$

From 12, 22 and 24 we see that:

$$
\Delta \Gamma_{K} \approx-2 \Delta m_{K} .
$$


The calculation of $p / q$ according to Equation 9 can then be done by defining a phase $\phi_{12}$ :

$$
\frac{M_{12}}{\Gamma_{12}} \equiv-\left|\frac{M_{12}}{\Gamma_{12}}\right| e^{i \phi_{12}}
$$

Since the $C P$ violating effects in the K-meson system are known to be small, it is useful to work in terms of a series expansion of the phase $\phi_{12}$, and to note that to first order,

$$
\begin{gathered}
\Delta m_{K}=2\left|M_{12}\right|, \\
\Delta \Gamma_{K}=-2\left|\Gamma_{12}\right|,
\end{gathered}
$$

which gives us:

$$
\frac{M_{12}}{\Gamma_{12}}=\frac{\Delta M_{K}}{\Delta \Gamma_{K}}\left(1+i \phi_{12}\right)
$$

We go on to note that indirect $C P$ violation has been observed in the K-meson system in complimentary ways:

1. Asymmetries in semi-leptonic $K_{L}^{0}$ decays measure the quantity:

$$
\delta(\ell)=\frac{\Gamma\left(K_{L}^{0} \rightarrow \pi^{-} \ell^{+} \nu\right)-\Gamma\left(K_{L}^{0} \rightarrow \pi^{+} \ell^{-} \nu\right)}{\Gamma\left(K_{L}^{0} \rightarrow \pi^{-} \ell^{+} \nu\right)+\Gamma\left(K_{L}^{0} \rightarrow \pi^{+} \ell^{-} \nu\right)},
$$

and have found [17]:

$$
\delta(\mu)=(3.04 \pm 0.25) \times 10^{-3}, \quad \delta(e)=(3.33 \pm 0.14) \times 10^{-3} .
$$

These are manifestations of $C P$ violation in mixing where we can write $\delta(\ell)$ as:

$$
\delta(\ell)=\frac{1-|q / p|^{2}}{1+|q / p|^{2}}
$$

2. Asymmetries in the two-pion channels:

$$
\eta_{00}=\frac{A\left(K_{L}^{0} \rightarrow \pi^{0} \pi^{0}\right)}{A\left(K_{S}^{0} \rightarrow \pi^{0} \pi^{0}\right)}
$$




$$
\eta_{+-}=\frac{A\left(K_{L}^{0} \rightarrow \pi^{+} \pi^{-}\right)}{A\left(K_{S}^{0} \rightarrow \pi^{+} \pi^{-}\right)}
$$

have also been measured [17]:

$$
\begin{aligned}
& \left|\eta_{00}\right|=(2.275 \pm 0.019) \times 10^{-3}, \\
& \left|\eta_{+-}\right|=(2.285 \pm 0.019) \times 10^{-3} .
\end{aligned}
$$

Given these measurements, it is possible to separate the contributions from different channels and construct two complex parameters $\varepsilon_{K}$ and $\varepsilon_{K}^{\prime}$ whose real and imaginary parts are sensitive to different types of $C P$ violation:

$$
\begin{aligned}
\varepsilon_{K} & \equiv \frac{1}{3}\left(\eta_{00}+2 \eta_{+-}\right) \\
\varepsilon_{K}^{\prime} & \equiv \frac{1}{3}\left(\eta_{00}-\eta_{+-}\right) .
\end{aligned}
$$

Specifically, $\mathcal{R} e\left(\varepsilon_{K}\right)$ measures $C P$ violation in mixing, while the $\mathcal{R} e\left(\varepsilon_{K}^{\prime}\right)$ measures $C P$ violation in decay.

Experimental results from $\mathrm{KTeV}$ and NA48 have found greater than zero results for the value of $\mathcal{R} e\left(\varepsilon_{K}^{\prime} / \varepsilon_{K}\right)$. Specifically the $\mathcal{R} e\left(\varepsilon_{K}^{\prime} / \varepsilon_{K}\right)=(20.7 \pm 2.8) 10^{-4}$ result from the $\mathrm{KTeV}$

experiment [12], and the $\mathcal{R} e\left(\varepsilon_{K}^{\prime} / \varepsilon_{K}\right)=(15.3 \pm 2.6) 10^{-4}$ result from the NA48 experiment [18] have ruled out the possibility of a "Superweak" [19] addition to the Standard Model.

The Superweak theory states that all $C P$ violating decays happen through a direct $\Delta S=$ 2 interaction in mixing. Since all $C P$ violation happens in mixing, we would expect that there would be no direct $C P$ violation. So, the $\mathrm{KTeV}$ and NA48 results of a non-zero direct $C P$ asymmetry clearly disproved the Superweak theory.

\subsubsection{The B Meson}

The neutral B-meson is the lightest meson that contains the $b$ quark $\left(B^{0}=d \bar{b}\right.$ and $\left.\overline{B^{0}}=\bar{d} b\right)$. Like the K-meson previously discussed, mass eigenstates are different from weak eigenstates. 
But instead of "short" and "long" lived states for the K-meson; the B-meson can be defined by "light" and "heavy" in terms of the flavor $(C P)$ eigenstates:

$$
\begin{aligned}
& \left|B_{L}\right\rangle=p\left|B^{0}\right\rangle+q\left|\bar{B}^{0}\right\rangle, \\
& \left|B_{H}\right\rangle=p\left|B^{0}\right\rangle-q\left|\bar{B}^{0}\right\rangle .
\end{aligned}
$$

With the complex coefficients $p$ and $q$ obeying the normalization condition:

$$
|p|^{2}+|q|^{2}=1
$$

Current experimental values for the mass, mass difference $\Delta m_{d}$, and width difference $\Delta \Gamma_{B}$ are $[20]:$

$$
\begin{gathered}
\Delta m_{d} \equiv M_{H}-M_{L}=.502 \pm .007 \times 10^{12} s^{-1}, \quad \Delta \Gamma_{B} \equiv \Gamma_{H}-\Gamma_{L} \\
M_{B}=5.279 .4 \pm 0.5 \mathrm{MeV}, \quad x_{d} \equiv \Delta m_{d} / \Gamma_{B}=0.771 \pm 0.012 .
\end{gathered}
$$

Beyond this, the formalism of the B-meson is almost the same as the K-meson.

\subsection{Penguin Decays}

In the Standard Model, B-meson decays are dominated by $b \rightarrow c$ transitions that occur at tree level through flavor changing charged currents. However, flavor changing neutral currents (FCNC) are absent at tree level. FCNC interactions are processes that change a quark of one charge to a different quark of the same charge and different flavor (i.e. a direct

$b \rightarrow s$ or $b \rightarrow d$ transition). Effective FCNC can take place through second order processes, represented by a "penguin diagram" [21].

From kaon decay, the difference in decay rates of neutral versus charged current interactions were seen to be quite large:

$$
\frac{K^{+} \rightarrow \pi^{+} \nu \bar{\nu}}{K^{+} \rightarrow \pi^{0} \mu^{+} \nu_{\mu}}<10^{-5}
$$


These measurements allowed Glashow, Iliopoulos, and Maiani (GIM) [22], to predict the existence of the $c$ quark years before it was discovered. They had reasoned that if there were only three quarks, then FCNC should be allowed at tree level. By including a fourth quark in the interactions, and separating the four quarks into two weak doublets, all tree level FCNC cancel in the decay rate calculation.

In Figure 4, we see the Feynman diagram of a $b \rightarrow s \gamma$ (b to s quark transition with a radiative photon) decay. The second order, effective flavor changing neutral current decay in which the $b \rightarrow s$ transition has two vertices has a much smaller decay amplitude than tree level transitions like $b \rightarrow c$. In these electromagnetic penguin decays, a charged particle emits an external real photon. The inclusive rate is dominated by short distance (weak) interaction and can be reliably predicted [23].
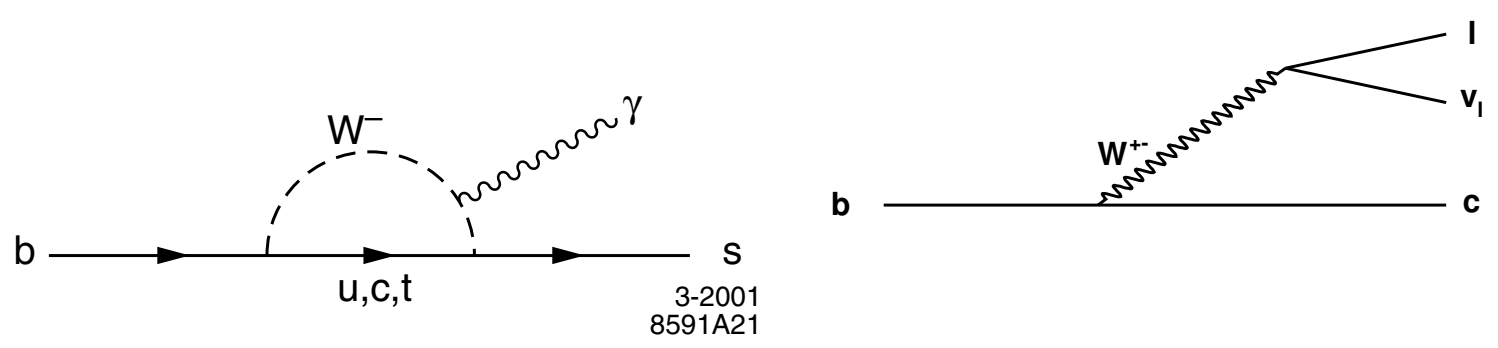

Figure 4: Example Feynman diagrams for a Penguin $b \rightarrow s \gamma$ decay (left) and a tree level $b \rightarrow c$ decay (right).

Calculating inclusive weak decay rates for B-mesons can be done using operator product expansion. At energy scales $\mu=m_{b} \ll M_{W}$ a set of "effective" local transition operators [24] is used. For an expansion coefficient, a heavy quark effective theory limit $m_{b} \rightarrow \infty$ is used such that all quantities are explicitly dependent on $m_{b}$ (i.e. the $b$ quark is not explicitly removed from the theory).

These effective theories start at a higher energy scale (for our case of $b \rightarrow s \gamma, \mu \sim M_{W}$ ), and then integrate out heavier degrees of freedom ( $W$ boson and $t$ quark) from explicitly 
appearing in the theory. The effects of the heavy degrees of freedom are not removed, but simply hidden in a set of effective gauge coupling constants and coefficients describing the effective strength at our energy scale $\mu$. The effective Hamiltonian is then:

$$
\mathcal{H}_{\text {eff }} \sim V_{t b} V_{t s}^{*} \sum_{i} C_{i}(\mu) Q_{i}(\mu) .
$$

$Q_{i}(\mu)$ denotes the local operators generated by strong and electroweak interactions, and $C_{i}(\mu)$ are the Wilson coefficient functions [24]. By integrating out the heavier degrees of freedom, the physics information is now contained within the Wilson coefficients. The general expression for the Wilson coefficients is:

$$
C_{i}(\mu)=U_{i}\left(\mu, M_{W}\right) C_{i}\left(M_{W}\right)
$$

where $C_{i}\left(M_{W}\right)$ is our initial state and $U_{i}\left(\mu, M_{W}\right)$ is the energy scale evolution matrix.

\section{$2.5 \quad A_{C P}$ in $B \rightarrow X_{S} \gamma$ Decay}

Current predictions of Standard Model direct $C P$ violation in $B \rightarrow X_{S} \gamma$ decays have been calculated using heavy quark effective theory [25]:

$$
A_{C P}^{s, d}=\frac{\Gamma\left(B \rightarrow X_{s, d} \gamma\right)-\Gamma\left(\bar{B} \rightarrow \bar{X}_{s, d} \gamma\right)}{\Gamma\left(B \rightarrow X_{s, d} \gamma\right)+\Gamma\left(\bar{B} \rightarrow \bar{X}_{s, d} \gamma\right)} \sim \operatorname{Im}\left(\epsilon_{s, d}\right)\left(C_{2} C_{8}-C_{2} C_{7}\right),
$$

where $C_{2}, C_{7}$, and $C_{8}$ are Wilson coefficients, while $\epsilon_{s, d}$ is:

$$
\epsilon_{s}=\frac{V_{u s}^{*} V_{u b}}{V_{t s}^{*} V_{t b}} \approx \lambda^{2}(i \eta-\rho)=O\left(10^{-2}\right),
$$

for $b \rightarrow s \gamma$ transitions, and:

$$
\epsilon_{d}=\frac{V_{u d}^{*} V_{u b}}{V_{t d}^{*} V_{t b}} \approx \frac{\rho-i \eta}{1-\rho+i \eta}=O(1)
$$


for $b \rightarrow d \gamma$ transitions. These next-to-leading order calculations estimate Standard Model $C P$ violation in $b \rightarrow s \gamma$ decays at [25]:

$$
A_{C P}=0.0044_{-0.0009}^{+0.0019}{ }_{-0.0003}^{+0.0003}{ }_{-0.0010}^{+0.0015}
$$

where the three errors are associated with the experimental and theoretical uncertainties in: $\alpha_{s}$, CKM matrix, and $\left(m_{c} / m_{b}\right)^{2}$.

But, amplification of direct $C P$ violation in $B \rightarrow X_{S} \gamma$ decays may arise from new physics contributions in the Wilson coefficient, with strong phases provided by the imaginary parts of the matrix elements of the effective Hamiltonian [25]. These imaginary parts are seen at $\mathcal{O}\left(\alpha_{s}\right)$ from loop diagrams containing light quarks or gluons, while the contributions to $A_{C P}\left(B \rightarrow X_{S} \gamma\right)$ from virtual corrections arise from interference of the one loop diagrams with $C_{7}$ and tree level diagrams with $C_{8}$ [25]. These amplifications could be due to $C P$ violating SUSY parameters (SUSY particles coupling to Standard Model particles could vary with the $C P$ state of the Standard Model particle), which enter into loop computations. Possible $C P$ violations of up to $15 \%$ [25] would be seen in radiative loop decays like $b \rightarrow s \gamma$ if the $\mathrm{W}$ boson (see Figure 4) is replaced by a charged Higgs, or if the $\mathrm{W}$ boson and $t$ quark were replaced with a chargino and an up-type squark or a neutralino with a down-type squark.

Similarly, $C P$ asymmetry in $b \rightarrow d \gamma$ decays can be computed [25]. It is found to be larger than the $b \rightarrow s \gamma C P$ asymmetry by a factor $\sim 20$ (due to the factors related to the CKM matrix elements). If only the sum of the $b \rightarrow s \gamma$ and $b \rightarrow d \gamma$ decays is measured, the $C P$ asymmetry vanishes (in the limit $m_{s}=m_{d}=0$ ) because of the unitarity of the CKM matrix [25].

The first measurement of an inclusive CP asymmetry was performed by CLEO [26]. They used two distinct methods of flavor tagging. One requires just the high-energy photon (between 2.2 and $2.7 \mathrm{GeV}$ ), and a lepton from the other $B$ to give the flavor tag. In this 
method the dominant source of mistagging is $B^{0}-\bar{B}^{0}$ mixing. The method does not distinguish between $b \rightarrow s \gamma$ and $b \rightarrow d \gamma$. The second method they use, "pseudo-reconstruction" of a possible $X_{s}$ system, where $X_{s}$ refers to inclusive strange hadronic states, which is selftagging, although they do not correct for cross-feed from other $b \rightarrow s \gamma$ decays in the way that we do. This method measures the asymmetry in $b \rightarrow s \gamma$ alone. Their final direct $C P$ asymmetry measurement is a weighted average of the two methods. The weighted average of the asymmetries in $b \rightarrow s \gamma$ and $b \rightarrow d \gamma$ is quoted from the CLEO collaboration as [26]:

$A_{C P(s+d)}=0.965 A_{C P}(b \rightarrow s \gamma)+0.02 A_{C P}(b \rightarrow d \gamma)=(-0.079 \pm 0.108 \pm 0.022)(1.0 \pm 0.030)$

The first (and by far largest) error is statistical, the second is an additive systematic, and the third a multiplicative systematic.

A more recent measurement of $A_{C P}$ was performed by the BELLE collaboration [27]. BELLE has released a conference paper containing a measurement of $A_{C P}$ in $B \rightarrow X_{S} \gamma$ decays. With a data sample of $140 \mathrm{fb}^{-1}$ they use 682 signal events to measure:

$$
A_{C P}^{B E L L E}=-0.004 \pm 0.051 \pm 0.038
$$

where the first error is statistical and the second is systematic. 


\section{The BABAR Experiment}

No software people need to be here...

The BABAR experiment is an asymmetric $e^{+} e^{-} \mathrm{B}$ factory at the Stanford Linear Accelerator Center (SLAC). Every year BABAR detects millions of $B \bar{B}$ pairs produced at the $\Upsilon(4 \mathrm{~S})$ resonance, allowing studies of $B$ decays and other bottom and charm dominated processes.

\section{$3.1 \quad$ PEP-II}

The PEP-II storage rings supply the BABAR detector with $e^{+} e^{-}$collisions. They operate at a center of mass energy of $10.58 \mathrm{GeV}$ directly corresponding to the $\Upsilon(4 \mathrm{~S})$ resonance. The $\Upsilon(4 \mathrm{~S})$ decays almost exclusively decays to $B \bar{B}$. The storage rings are composed of a $9 \mathrm{GeV}$ high energy electron ring, and a $3.1 \mathrm{GeV}$ low energy positron ring.

The electrons and positrons are originally created and accelerated in the SLAC Linac (see Figure 5), then injected into the PEP-II ring for collision. The asymmetric energies of the electron-positron collision, boost the produced $\Upsilon(4 \mathrm{~S})$ in the lab frame to a $\beta \gamma=0.56$ in the "forward" direction. This boost causes an average distance of $300 \mu \mathrm{m}$ between the two $B$ decay vertices, and allows for accurate measurements of time dependent $C P$ processes.

Current luminosities in the PEP-II rings allow for the collection of $500 \mathrm{pb}^{-1}$ of data per day. This corresponds to about half a million produced $B \bar{B}$ events per day, with a peak instantaneous luminosity of $>7.7 \times 10^{33} \mathrm{~cm}^{-2} \mathrm{~s}^{-1}$. The effective cross-section production of fermion pairs at the $\Upsilon(4 \mathrm{~S})$ resonance can be seen in table 2 . We note that $b \bar{b}$ production is less than a quarter of total quark pair production, and only about $2 \%$ of the total cross-section.

\subsection{The BABAR Detector}

The BABAR detector is pictured in Figure 6. The detector is made up of 5 subsystems: a silicon vertex tracker (SVT), a drift chamber $(\mathrm{DCH})$, the detector of internally reflected 


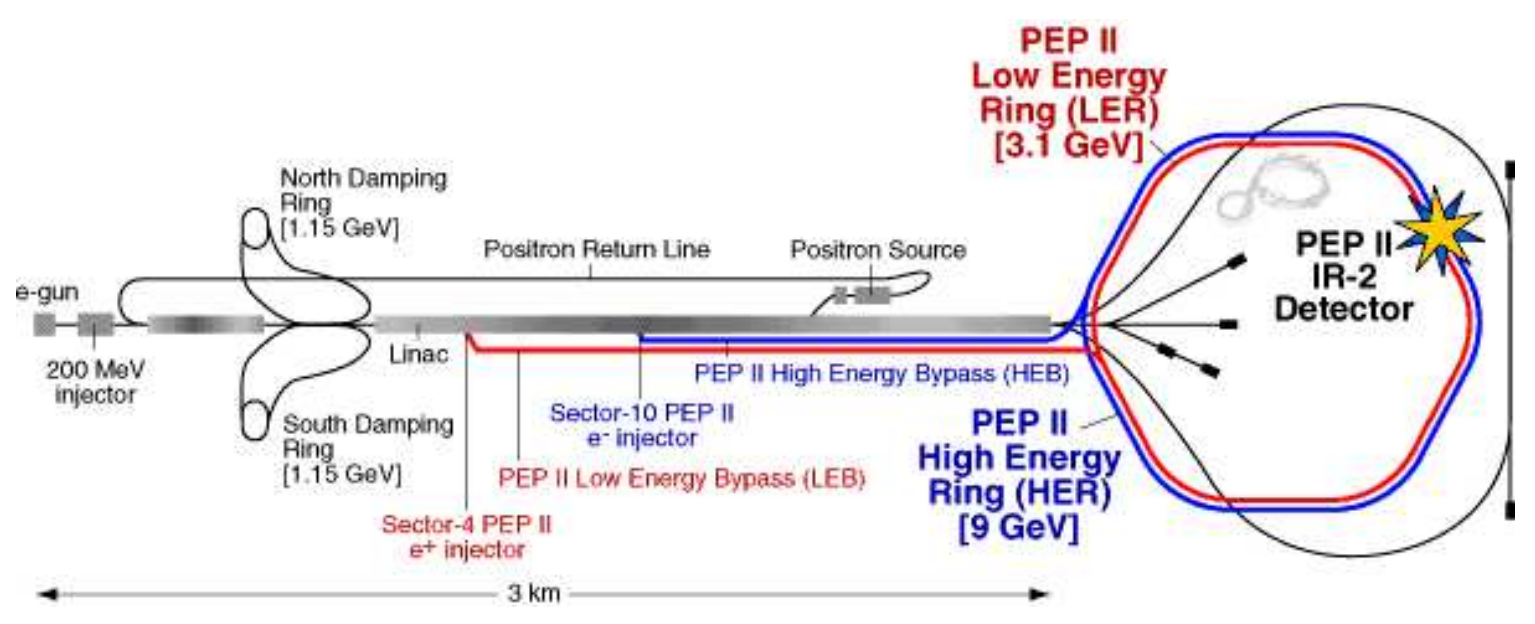

Figure 5: Schematic of SLAC, PEP-II, and placement of the BABAR detector.

\begin{tabular}{|c|c|}
\hline$e^{+} e^{-} \rightarrow$ & Cross-section (nb) \\
\hline$b \bar{b}$ & 1.05 \\
$c \bar{c}$ & 1.30 \\
$s \bar{s}$ & 0.35 \\
$u \bar{u}$ & 1.39 \\
$d \bar{d}$ & 0.35 \\
\hline$\tau^{+} \tau^{-}$ & 0.94 \\
$\mu^{+} \mu^{-}$ & 1.16 \\
$e^{+} e^{-}$ & $\sim 40$ \\
\hline
\end{tabular}

Table 2: Production cross-section at $\Upsilon(4 \mathrm{~S})$ resonance for $B A B A R$. 
Cherenkov light (DIRC), an electromagnetic calorimeter (EMC), and an instrumented flux return (IFR). Between the EMC and IFR there is a super conducting solenoid, that creates a uniform 1.5 Tesla magnetic field in the SVT, DCH, DIRC, and EMC.

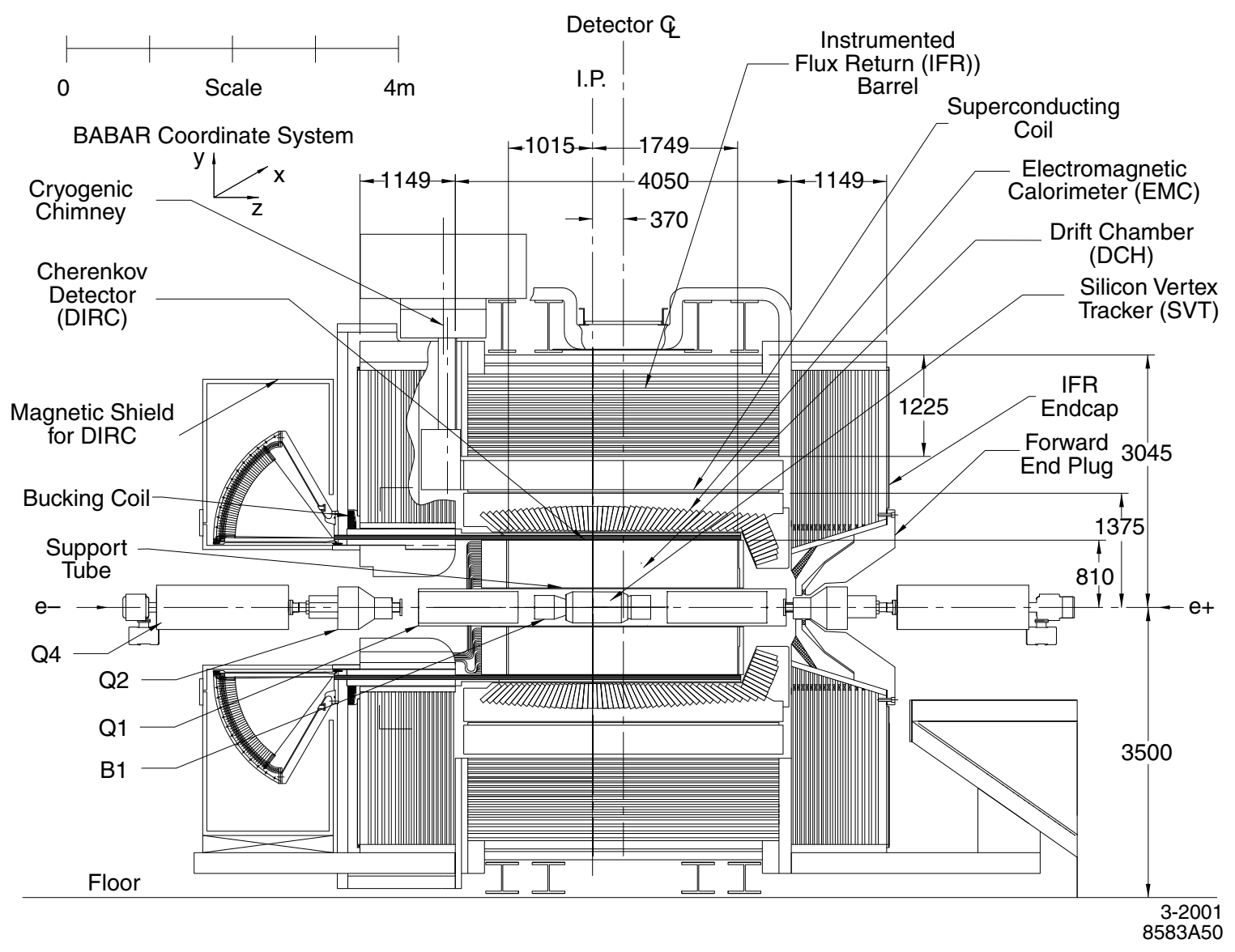

Figure 6: BABAR detecor longitudinal view.

\subsubsection{Silicon Vertex Detector (SVT)}

The SVT is comprised of five layers of double sided silicon strips at 32, 40, 54, 91-127, and 114-144 millimeters away from the interaction point (see Figure 7). The first three layers of the SVT are placed as close as possible to the interaction point to reduce scattering effects from the beryllium beam pipe. The outer two layers are used to help associate charged 
tracks reconstructed by the SVT, with charged tracks reconstructed by the drift chamber (see section 3.2.2).

The geometrical acceptance of the SVT is from $20.1^{\circ}$ to $-29.8^{\circ}$ (polar angle). This covers about a $90 \%$ of the solid angle in the center of mass or $\Upsilon(4 \mathrm{~S})$ frame. Typically, $86 \%$ of the solid angle is used for charged particle reconstruction due to edge effects.

The SVT provides position and pulse height information. Calibrated time over noise threshold allows for $d E / d x$ measurements of charged tracks. SVT only $d E / d x$ measurements can be used to separate pions and kaons for momenta up to $0.5 \mathrm{GeV}$.

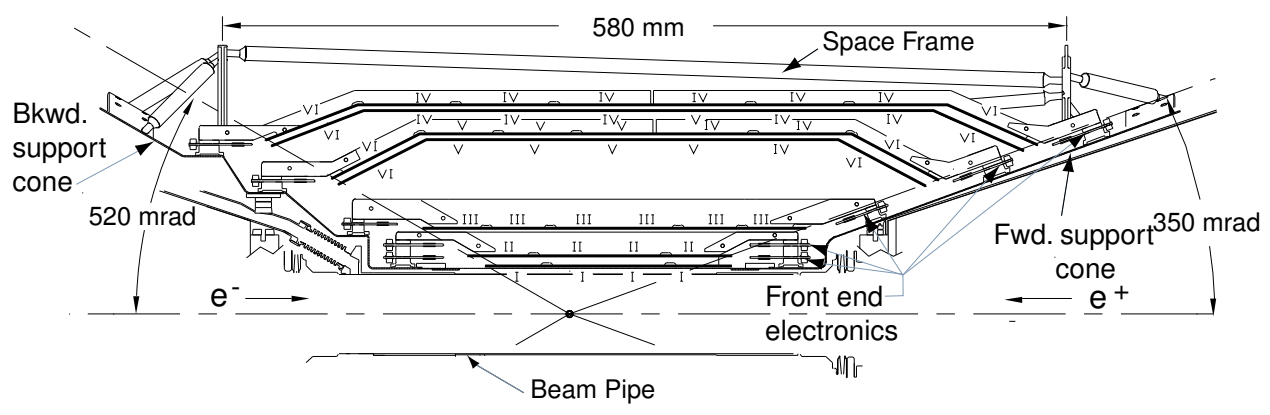

Figure 7: Longitudinal schematic view of the SVT.

\subsubsection{Drift Chamber (DCH)}

The BABAR drift chamber is made of 40 layers of hexagonal cells, with one sense wire in the center of six field wires. For better longitudinal accuracy, 24 of the 40 layers are placed at small angles relative to the longitudinal direction (see Figure 7). The wires sit in an 80:20 helium:isobutane gas mixture. The inner and forward walls of the drift chamber are kept as thin as possible to reduce the number of interaction lengths between detector subsystems components. 
The drift chamber has a $d E / d x$ resolution of $7.5 \%$ for separation of low momentum particles. In Figure 9, we can see good pion/kaon separation for momentum less than 0.7 $\mathrm{GeV}$.

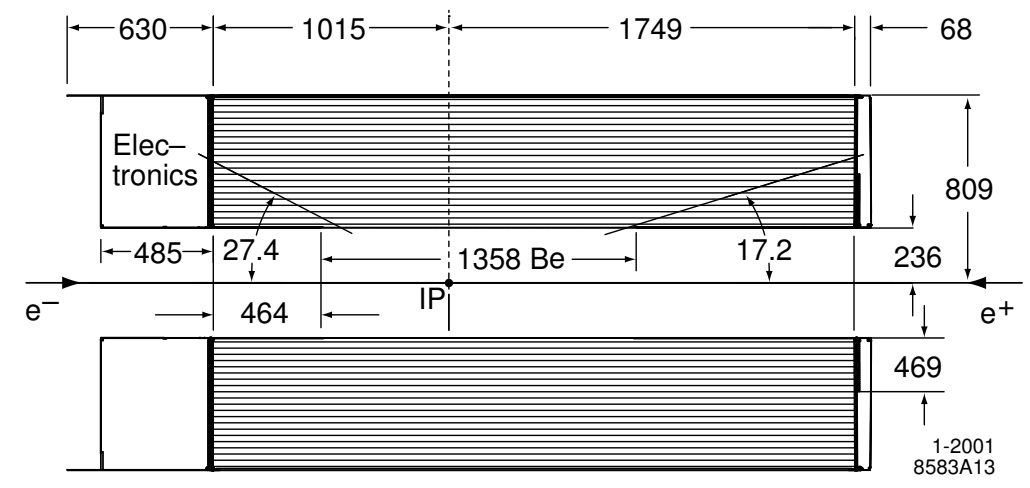

Figure 8: Longitudinal schematic view of the DCH. The chamber is offset by 0.37 meters from the interaction point (in the forward direction).

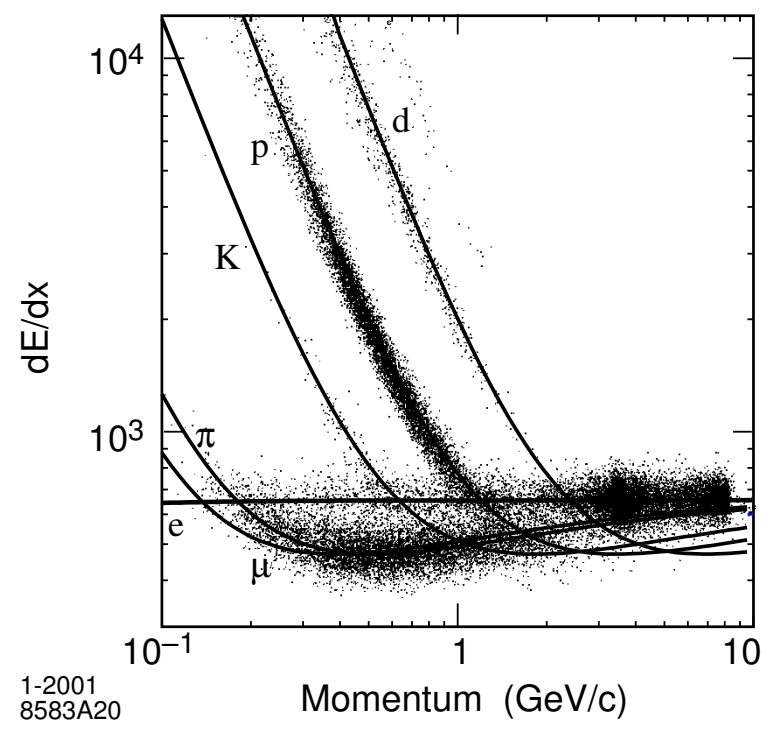

Figure 9: Plot of $d E / d x$ in the DCH as a function of track momentum for different particle types. 


\subsubsection{Detector of Internally Reflected Cherenkov Light (DIRC)}

The DIRC is an innovative detector made up of fused silica bars that are placed between the DCH and EMC (see section 3.2.4). When particles pass through the DIRC, Cherenkov photons are transmitted down the bars via total internal reflection. One end of the bar is mirrored, while the other end is connected to a hemispherical, water-filled container called the "Stand Off Box" (see Figure 10). The outside edge of the standoff box is covered by an array of photomultiplier tubes (PMT) for readout.

The DIRC is an intrinsically three-dimensional imaging device. The light patterns on the PMT's from the initial and reflected wave fronts are conic sections. By measuring the photon propagation angles (by knowing the position of the struck PMT's), we can define the direction the particle was moving. From the incident time difference of the two wave fronts, we can define where on the quartz bar the particle was incident. From the photon propagation angles, we can calculate the Cherenkov angle for an assumed particle hypothesis. The improvement can be seen for kaons in Figure 11.

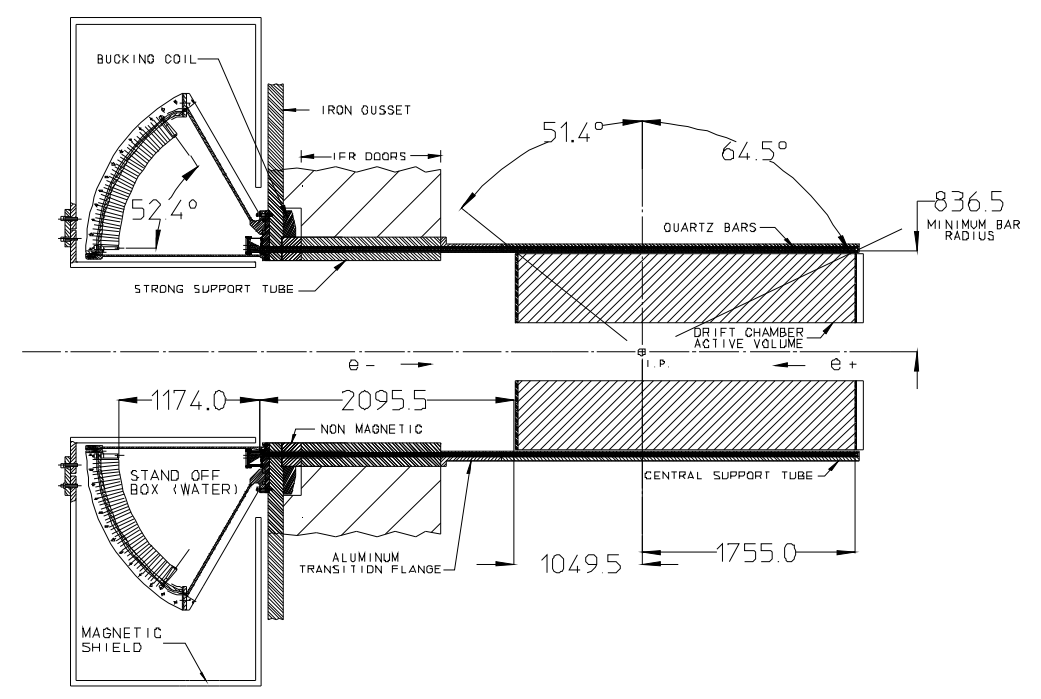

Figure 10: Schematic view of the DIRC. 


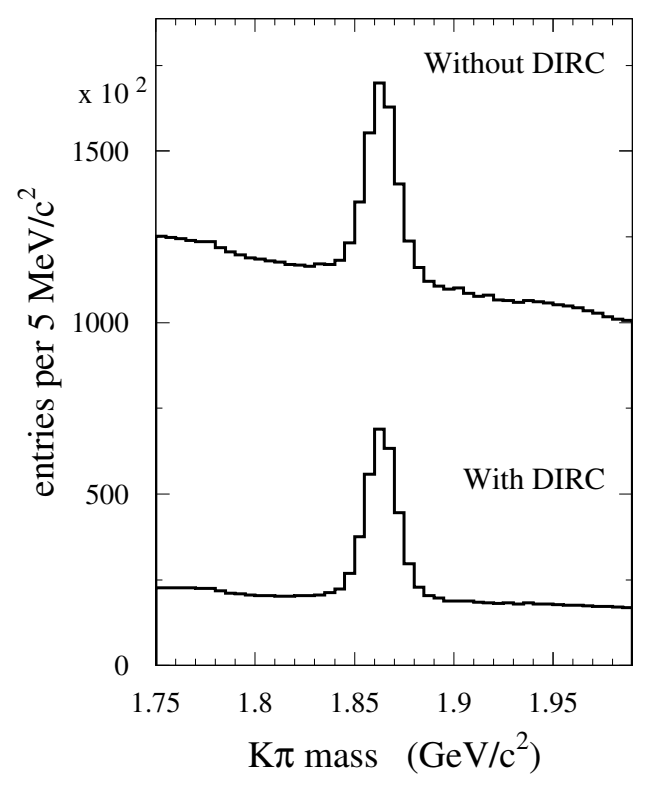

Figure 11: Invariant $\mathrm{K} \pi$ inclusive mass spectrum with and without the use of the DIRC for kaon identification. The mass peak corresponds to the decay of the $D^{0}$ meson.

\subsubsection{Electromagnetic Calorimeter (EMC)}

The BABAR EMC is designed to measure electromagnetic showers above $20 \mathrm{MeV}$ with good energy and angular resolution. The EMC is comprised of 6580 thallium doped cesium iodide (CsI(T1)) crystals. The crystals are read out with silicon photodiodes.

The EMC consists of a cylindrical barrel and forward end cap (see Figure 12). The coverage is from $15.8^{\circ}$ to $141.8^{\circ}$, which corresponds to $90 \%$ of the solid angle in the $\Upsilon(4 \mathrm{~S})$ system.

When a particle strikes the EMC, the corresponding ionization or electromagnetic shower normally spans multiple crystals. This energy deposit is called a "cluster." Since multiple particles can strike a single area, multiple local maxima ("bumps") can arise. Reconstruction algorithms try to match DCH tracks with the cluster centroids (energy weighted center) using $\chi^{2}$ from the expected impact points. All unmatched clusters are treated as neutral particle 


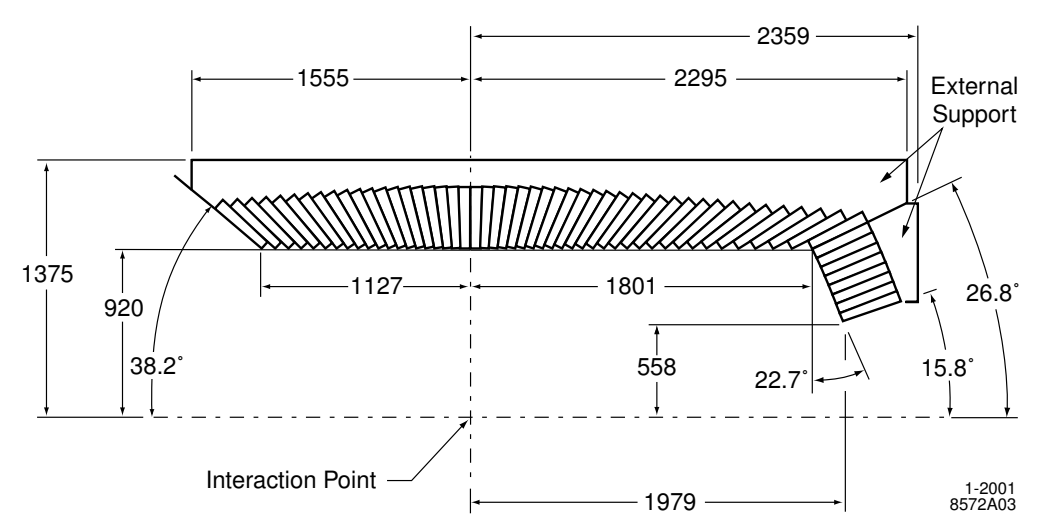

Figure 12: Schematic view of the EMC.

candidates.

In this analysis, EMC angular and energy resolution are very important for reconstructing the correct photon energy and $X_{S}$ mass. Plots of the angular and energy resolution for the calorimeter can bee seen in Figure 13. Energy resolution in our photon energy range is calibrated from Bhabha scattering. The fit energy resolution as a function of photon energy is:

$$
\frac{\sigma_{E}}{E}=\frac{(2.32 \pm 0.30) \%}{\sqrt[4]{E(\mathrm{GeV})}} \oplus(1.85 \pm 0.12) \%
$$

Angular resolution is measured using symmetric $\pi^{0}$ and $\eta$ decays. The resolution varies between $3 \mathrm{mrad}$ at high energies, to $12 \mathrm{mrad}$ at low energies:

$$
\sigma_{\theta}=\sigma_{\phi}=\frac{(3.87 \pm 0.07)}{\sqrt{E(\mathrm{GeV})}} \pm 0.04 \mathrm{mrad}
$$

\subsubsection{Particle Identification}

For our analysis, we are mainly interested in kaons, pions, and photons. For charged kaons and pions, the SVT, DCH, and DIRC are used. Tracks formed in the SVT are matched to tracks formed in the DCH. The $d E / d x$ information (see figure 9 ) from the DCH is then 

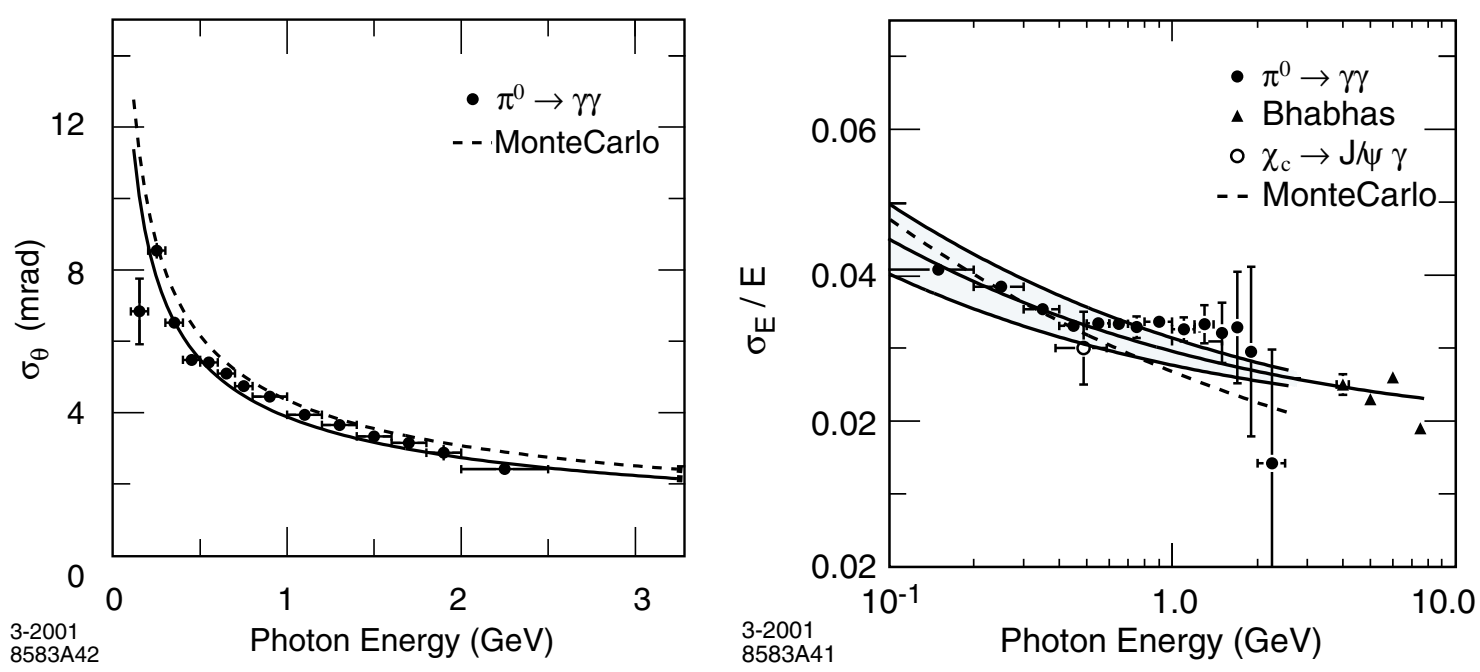

Figure 13: Angular(left) and energy(right) resolution of the EMC for photons coming from neutral pions.

used to determine particle ID. However, if no track matching is found between the SVT and DCH, then the SVT has a stand-alone particle ID algorithm.

For high momentum tracks (momentum $>0.7 \mathrm{GeV}$ ), the DIRC also provides particle ID from Cherenkov angle calculations. Separation for pions and kaons is about $4.2 \sigma$ at $3 \mathrm{GeV}$ (see improvement in kaon identification in Figure 11).

Excellent angular and energy resolution in the EMC is needed for accurate neutral pion and photon reconstruction. In Figure 13, we can see the angular and energy resolution for photons from neutral pions.

\subsubsection{Instrumented Flux Return (IFR)}

The IFR is designed to be a detector of muons and neutral hadrons (primarily $K_{L}^{0}$ and neutrons) over a large momentum range. It consists of 18 layers of resistive plate chambers (RPC) in the end caps, and 19 layers in the barrel region. Interspersed between the RPC's layers are steel layers that act as a magnetic flux return and hadron absorber. The thickness 
of the steel layers is graduated from a minimum of $2 \mathrm{~cm}$ (inner most layer) to a maximum 10 $\mathrm{cm}$ (outer most layer). The gradation of the steel allows for better neutral hadron detection in the inner most layers.

The RPC's are a cost effective way of instrumenting the IFR. They consist of a $2 \mathrm{~mm}$. gap that is enclosed by Bakelite (there are spacers to keep the desired thickness). The Bakelite is covered by a graphite film, then insulator, then readout strips (see Figure 14). When an ionizing particle passes through the RPC, the resultant streamer depletes the local charge on the Bakelite, and is detected by the readout strips.

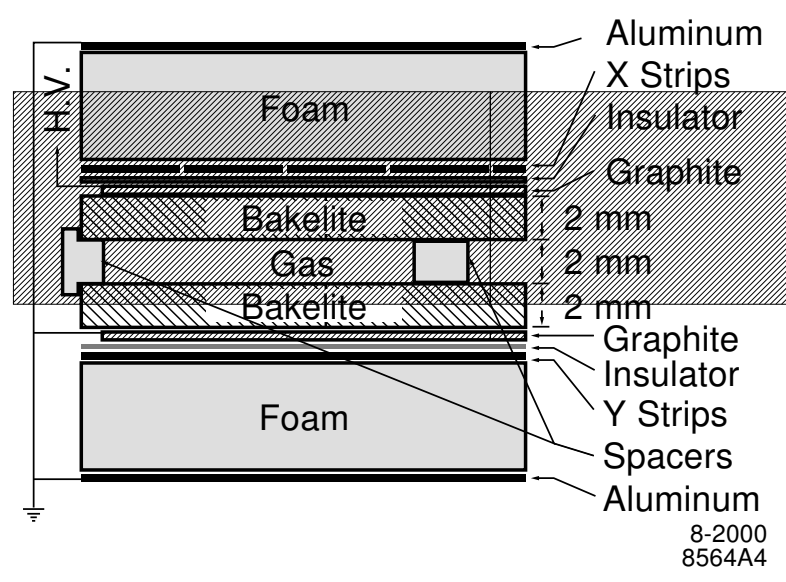

Figure 14: Longitudinal schematic view of the the IFR RPC's. 


\section{Monte Carlo and Data Samples}

Couldn't we set the chamber efficiency to a .tcl parameter?

We rely on Monte Carlo (simulated decays and detection of the decays including detector performance) for extracting our cut points, signal efficiency, and fitting technique. We can cross check our Monte Carlo with non-signal samples (explained later), but important information like peaking background (background that is indistinguishable from signal) can only be estimated from Monte Carlo. We use fourth generation BABAR Monte Carlo samples, and the data sample from November 1999 to June 2002 (RUNs 1 and 2).

The $B \rightarrow X_{s} \gamma$ signal model is described in reference [28]. The model breaks the hadronic mass $\left(X_{s}\right)$ spectrum into two parts: the resonant $K^{*}$ region, and the non-resonant region. The resonant $K^{*}$ region is defined as $M_{X s}<1.1 \mathrm{GeV}$, and is modeled with exclusive $K^{*}$ decays. The non-resonant region $\left(M_{X s}>1.1 \mathrm{GeV}\right)$ is modeled by using Heavy-Quark Effective Theory. In 1998 Kagan and Neubert calculated next-to-leading order [28] decay rate for the non-resonant region of $B \rightarrow X_{s} \gamma$ decays.

Kagan and Neubert adopt an exponential distribution that depends on just the first and second moment of the spectra, i.e. the width of the distribution. The formula is:

$$
F\left(k_{+}\right)=(1-x)^{a} e^{(1+a) x}, \quad x=\frac{k_{+}}{\bar{\Lambda}} \leq 1,
$$

where $\bar{\Lambda}=m_{B \text { meson }}-m_{b \text { quark }}, a$ can be related to the second moment, and $F\left(k_{+}\right)$is the $b$ quark momentum distribution in the $\mathrm{B}$ meson. The two free parameters $\bar{\Lambda}$ and $a$ are preferably written as a function of the effective b-quark mass, $m_{b}$ (sensitive to the mean photon energy or first moment), and a parameter, $\lambda_{1}$ (directly proportional to the second moment). The parameters $\lambda_{1}$ and $m_{b}$ should be seen as independent, although naively it might be expected that $\lambda_{1}$ increases as $m_{b}$ decreases. Reasonable ranges for the two 
parameters are: 4.55 to $4.95 \mathrm{GeV}$ for $m_{b}$ and -0.5 to $-0.2 \mathrm{GeV}^{2}$ for $\lambda_{1}$. It is important to note that the spectrum does not show a strong dependence on the choice of the shape function, and that any $C P$ asymmetry seen is not dependent on the spectral shape [25]. Our various data sets used are listed below:

- Inclusive $B \rightarrow X_{s} \gamma$ signal Monte Carlo events

- The equivalent of $269 \mathrm{fb}^{-1}$ of events (assuming $\mathrm{BF}(b \rightarrow s \gamma)=3.5 \times 10^{-4}$ ) are generated using the Kagan and Neubert [28] modeling with parameters $m_{b}=4.80$ $\mathrm{GeV}$ and $\lambda_{1}=-0.30 \mathrm{GeV}^{2}(\mathbf{K N} 480)$ and an exponential shape function.

- A total of 1.13 million events generated with a flat $E_{\gamma}$ spectrum in the range 1.6 to $2.7 \mathrm{GeV}$ (0.6 to $3.3 \mathrm{GeV}$ in $M_{X s}$ ). We can weight this sample to match any predicted set of parameters. We use $m_{b}=4.80 \mathrm{GeV}$ and $\lambda_{1}=-0.30 \mathrm{GeV}^{2}$ unless explicitly written.

The hadronic $s \bar{d}$ and $s \bar{u}$ systems are fragmented into multi-body final states using the default settings of JETSET [29]. The fragmentation can be checked by comparing the relative final state yields observed in data with those generated in the inclusive Monte Carlo. By weighting the different final states in the Monte Carlo to match the distribution in data, it is possible to obtain a better match of the total Monte Carlo sample to the data.

From a technical point of view, in order to separate the signal and cross-feed samples (in the Monte Carlo) we use a Monte Carlo truth-matching (Truth matching is matching reconstructed Monte Carlo decay to the generated Monte Carlo decay.). For the signal sample we require that the true (generated) and reconstructed decay mode are the same, and that all reconstructed daughters match Monte Carlo generated particles: 
- All reconstructed final state daughters are matched to the true final state daughters.

- The difference between the true and the reconstructed hadronic mass is within $50 \mathrm{MeV}$.

For cross-feed the equivalent definitions are:

- The true and reconstructed decay mode are the same, and all daughters are truth matched, but the difference between the true and the reconstructed hadronic mass is larger than $50 \mathrm{MeV}$.

- The true and reconstructed decay mode are not the same.

- Not all daughters are truth matched to Monte Carlo particles.

More details about the truth-match are given in the Appendix A. Note again that the iType mapping does not depend on the $b$ flavor. This means that having the reconstructed mode identical to the true mode allows the reconstructed $b$ flavor to be different from the generated $b$ flavor. This case is covered in the systematic Appendix D.1.

\section{- $\underline{B \rightarrow K^{*}(892) \gamma \text { signal Monte Carlo events }}$}

The equivalent of $3656 \mathrm{fb}^{-1}$ of resonant $B \rightarrow K^{*}(892) \gamma$ signal Monte Carlo (assuming $\left.\mathrm{BF}\left(B \rightarrow K^{*} \gamma\right)=4.0 \times 10^{-5}\right)$ are generated in the following final states:

$$
\begin{aligned}
& -K^{* 0} \rightarrow K^{+} \pi^{-} . \\
& -K^{* 0} \rightarrow K_{S} \pi^{0}, K_{S} \rightarrow \pi^{+} \pi^{-} . \\
& -K^{*+} \rightarrow K^{+} \pi^{0} . \\
& -K^{*+} \rightarrow K_{S} \pi^{+}, K_{S} \rightarrow \pi^{+} \pi^{-} .
\end{aligned}
$$


We do not include any modes with a $K_{L}$, nor do we allow the $K_{S}$ to decay to two $\pi^{0}$ 's. In order to simplify the interpretation of our results, we correct the signal efficiency to account for the full $K_{S}$ decays, i.e. include the $K_{S} \rightarrow \pi^{0} \pi^{0}$ decays. This entails reducing the signal efficiency by a factor calculated from isospin considerations. Similarly, we often write $K^{0}$. This assumes that the $K_{L}$ contribution is already included.

- $\underline{c \bar{c} \text { Monte Carlo events }}$

$c \bar{c}$ Monte Carlo events are defined as simulated $e^{+} e^{-} \rightarrow c \bar{c}$ quark pairs. $102 \mathrm{fb}^{-1}$ of $c \bar{c}$ Monte Carlo is generated, assuming $\sigma=1.3 \mathrm{nb}$ (133 million $c \bar{c}$ Monte Carlo events).

- $\underline{u d s}$ Monte Carlo events

$u d s$ Monte Carlo events are defined as simulated $e^{+} e^{-} \rightarrow u \bar{u}, d \bar{d}$, and $s \bar{s}$ quark pairs. $99.8 \mathrm{fb}^{-1}$ of $u d s$ Monte Carlo is generated, assuming $\sigma=2.09 \mathrm{nb}$ (209 million $u d s$ Monte Carlo events).

- $\underline{B \bar{B}}$ Monte Carlo events

$B \bar{B}$ Monte Carlo events are defined as simulated $e^{+} e^{-} \rightarrow B \bar{B}$, where the B-mesons do not decay to an $X_{S}$ or $K^{*} .200 \mathrm{fb}^{-1}$ of $B \bar{B}$ Monte Carlo is generated, assuming $\sigma=1.09 \mathrm{nb}(218$ million $B \bar{B}$ Monte Carlo events $)$.

- $\underline{\text { Off-resonance data }}$

$9.6 \mathrm{fb}^{-1}$ of off-resonance (data taken $40 \mathrm{MeV}$ below the $\Upsilon(4 \mathrm{~S})$ resonance) data is used.

- $\underline{\text { On-resonance data }}$

$81.9 \mathrm{fb}^{-1}$ of on-resonance data, which corresponds to $(88.9 \pm 1.0) \times 10^{6} B \bar{B}$ events, is used. 


\section{Analysis Strategy}

It was good enough for the Italian Renaissance masters.

There are three approaches to measuring $A_{C P}$ in $B \rightarrow X_{S} \gamma$ decays: Exclusive, SemiInclusive, and Inclusive.

Exclusive : Reconstruct each individual decay mode and look at the $A_{C P}$ in each individual decay mode.

Semi-Inclusive : Reconstruct each individual decay mode and look at the $A_{C P}$ in the sum of all decay modes.

Inclusive : Do not reconstruct the decay, but use photon and event shape information to define the decay.

Each approach has advantages and disadvantages which are listed in table 3.

\begin{tabular}{|c|c|c|}
\hline Exclusive & Semi-Inclusive & Fully Inclusive \\
\hline \hline Reconstruct single states & Reconstruct finite number of states & Do not reconstruct state \\
\hline High model dependence & Mid model dependence & Low model dependence \\
\hline More handles to reduce background & More handles to reduce background & Less constraints to reduce background \\
\hline- & Measure hadronic spectrum & $\begin{array}{c}\text { Measured photon spectrum smeared } \\
\text { by the B motion in the CM frame }\end{array}$ \\
\hline $3-6 \mathrm{MeV}$ resolution & $3-6 \mathrm{MeV}$ resolution & $50-100 \mathrm{MeV}$ resolution \\
\hline
\end{tabular}

Table 3: Advantages and disadvantages for the different ways to measure $A_{C P}$.

For an $A_{C P}$ measurement, the largest error (aside from the statistical error) comes from model dependence. The QCD factorization for the $X_{s}$ is only known to $10 \%$, so the larger the portion of the $X_{s}$ reconstructed, the smaller the theoretical error. It is also questionable as to whether one can measure a $C P$ asymmetry without looking at a large enough portion of the $X_{s}$ decay [30]. 
We decided to use the Semi-Inclusive approach to extract $A_{C P}$. We reconstruct 12 self tagging $B \rightarrow X_{S} \gamma(b \rightarrow s \gamma)$ decays. To tag the $C P$ of the signal $B$, we either use the charge of the kaon, or the net pion charge.

These decays are extracted from the data by a series of cuts on particle quality and event shape parameters. The final distributions are fitted as function of reconstructed B-meson mass. Finally, the yields of the two $C P$ states are used to calculate the $A_{C P}$ for the sum of our 12 modes, where the final equation for calculating $A_{C P}$ from our signal yields is:

$$
A_{C P}=\left(A_{C P}^{\text {measured }}-\frac{\Delta D}{2}\right) \frac{1}{<D>}-A_{C P}^{\text {Shift }}
$$

Where D (the dilution factor) is defined as:

$$
\begin{aligned}
& D=1-2 \omega \\
& \bar{D}=1-2 \bar{\omega}
\end{aligned}
$$

and the average and difference of the dilution are:

$$
\begin{gathered}
<D>=\frac{D+\bar{D}}{2}, \\
\Delta D=D-\bar{D} .
\end{gathered}
$$

In order to define the $C P$ asymmetry, we first need calculate the mistag rate (see Appendix D.1), or the probability $\omega(\bar{\omega})$ that a $b(\bar{b})$ flavor is reconstructed as $\bar{b}(b)$ flavor. Thus, we define the dilution of the two flavors as in terms of the observables, $\omega$ and $\bar{\omega}$ :

$$
\begin{gathered}
<D>=1-(\omega+\bar{\omega}), \\
\Delta D=2 \times(\bar{\omega}-\omega) .
\end{gathered}
$$

Finally $A_{C P}^{\text {measured }}$ is defined as:

$$
A_{C P}^{\text {measured }}=\frac{n(b \rightarrow s \gamma)-n(\bar{b} \rightarrow \bar{s} \gamma)}{n(b \rightarrow s \gamma)+n(\bar{b} \rightarrow \bar{s} \gamma)}
$$


where $n(b \rightarrow s \gamma)$ and $n(\bar{b} \rightarrow \bar{s} \gamma)$ are the numbers of data events in the $b \rightarrow s \gamma$ and $\bar{b} \rightarrow \bar{s} \gamma$ final states respectively, and the $A_{C P}^{S h i f t}$ (the detector bias in measuring $A_{C P}$ ) is covered in Section 9.1.

The exclusive $b \rightarrow s \gamma$ decays are identified in the 16 final states listed in Table 4 . Note that out of these final states, the four $B$ to $K_{S}$ decays are mixed-flavor $C P$ states which are not used for the direct $C P$ violation measurement. These decays are kept in the analysis to check our understanding of $s$ and spectator quark fragmentation, and of the cross-feed among different final states. We note that the numbers in the tables and plots refer to only the 12 final states used for the $A_{C P}$ measurement, except in Appendix D.6.

These hadronic final states do not correspond to all the possible final states into which the $b$ quark system fragments. Table 4 shows the expected fraction of all final states to which the identified final states correspond, as a function of the effective $b$ quark mass. The $C P$ asymmetry is not very sensitive to the $b$ quark mass or the details of the fragmentation, but it is still desirable to use a significant fraction of the total rate for an inclusive measurement.

We determine from Monte Carlo (simulation) that we study a total of $53.2 \%(56.9 \%)$ final states for $m_{b}=4.65 \mathrm{GeV}\left(m_{b}=4.80 \mathrm{GeV}\right)$ within $M_{X s} \leq 2.3 \mathrm{GeV}$ (The signal model used is described in Section 4). If we assume that we have the same number of $K_{L}$ 's as $K_{S}$ 's, then the fraction investigated correspond to $70.9 \%(75.7 \%)$ of the final states for $m_{b}=$ $4.65 \mathrm{GeV}\left(m_{b}=4.80 \mathrm{GeV}\right)$.

To suppress the $B \bar{B}$ background (B-mesons that do not decay to an $X_{S}$ ), we require the $X_{S}$ mass to be $<2.3 \mathrm{GeV}$ (see Section 6.7), corresponding to a minimum photon energy, $E_{\gamma}>2.18 \mathrm{GeV}$, in the $B$ reference system. As mentioned in Section 4 , the $C P$ asymmetry should not be very sensitive to this cut.

We suppress the background from continuum (non $\Upsilon(4 \mathrm{~S})$ decays) events using a neural network based on event shape variables. Most of the continuum background comes from 


\begin{tabular}{|l|c|c|l|c|c|}
\hline \multirow{2}{*}{ Modes } & \multicolumn{2}{|c|}{$m_{b}(\mathrm{GeV})$} & \multirow{2}{*}{} & \multicolumn{2}{|c|}{$m_{b}(\mathrm{GeV})$} \\
\cline { 2 - 3 } \cline { 5 - 6 } & 4.65 & 4.80 & Modes & 4.65 & 4.80 \\
\hline \hline$B^{0} \rightarrow X_{S} \gamma, X_{S} \rightarrow$ & \multicolumn{2}{|c|}{ Fraction $(\%)$} & $B^{ \pm} \rightarrow X_{S} \gamma, X_{S} \rightarrow$ & \multicolumn{2}{|c|}{ Fraction (\%) } \\
\hline$K^{+} \pi^{-}$ & 8.4 & 10.7 & $K_{S} \pi^{+}$ & 4.2 & 5.3 \\
$K_{S} \pi^{0}$ & 2.3 & 2.9 & $K^{+} \pi^{0}$ & 4.5 & 5.7 \\
$K_{S} \pi^{+} \pi^{-}$ & 5.4 & 6.5 & $K^{+} \pi^{-} \pi^{+}$ & 10.8 & 13.0 \\
$K^{+} \pi^{-} \pi^{0}$ & 13.3 & 15.8 & $K_{S} \pi^{+} \pi^{0}$ & 6.6 & 7.8 \\
$K_{S} \pi^{0} \pi^{0}$ & 1.1 & 1.3 & $K^{+} \pi^{0} \pi^{0}$ & 2.3 & 2.7 \\
$K^{+} \pi^{-} \pi^{+} \pi^{-}$ & 3.2 & 3.3 & $K_{S} \pi^{+} \pi^{-} \pi^{+}$ & 1.6 & 1.6 \\
$K_{S} \pi^{0} \pi^{+} \pi^{-}$ & 4.7 & 4.9 & $K^{+} \pi^{-} \pi^{+} \pi^{0}$ & 9.3 & 9.8 \\
$K^{+} \pi^{-} \pi^{0} \pi^{0}$ & 2.8 & 2.8 & $K_{S} \pi^{+} \pi^{0} \pi^{0}$ & 1.4 & 1.4 \\
\hline Total & 41.2 & 48.1 & Total & 40.7 & 47.3 \\
\hline
\end{tabular}

Table 4: Studied $X_{S}$ final states and their rates (in percent) according to the Kagan and Neubert signal model [28] for two different values of the effective $b$ quark mass, $m_{b}$. The dependence of the fractions on the $b$ quark mass is a result of the different hadronic mass distributions. Note that $K_{S}$ includes the decay mode $K_{S} \rightarrow \pi^{0} \pi^{0}$, and that no constraint is made on $E_{\gamma}$

initial state radiation, and $D^{(*)}$ meson decays.

Most events have more than one $B$ candidate, and in these cases we select the candidate with the lowest value of $\left|\Delta E^{*}\right|\left(\left|\Delta E^{*}\right|=E_{B}^{*}-(\sqrt{s} / 2)\right.$, or the energy difference between the $B$ candidate and beam energy.). For events in which the "true" candidate has been reconstructed, the best candidate selection is about $90 \%$ correct. However, there are many events in which the true candidate is not reconstructed. "Fake" candidates are collectively known as $b \rightarrow s \gamma$ "cross-feed." Cross-feed candidates are candidates that: are reconstructed incorrectly, have the wrong $X_{S}$ mass, or the wrong final state is reconstructed. We evaluate cross-feed contributions using signal Monte Carlo samples, and correct for them in our analysis.

The continuum, $B \bar{B}$ and cross-feed backgrounds are subtracted by fitting the beamsubstituted mass distribution, $m_{\mathrm{ES}}\left(m_{\mathrm{ES}}=\sqrt{(\sqrt{s} / 2)^{2}-p_{B}^{* 2}}\right.$, or the the beam energy substituted mass.), to an Argus and a Crystal Ball (see appendix B) line shape. There are small 
components of the $B \bar{B}$ and cross-feed backgrounds that peak in $m_{\mathrm{ES}}$ (peaking background), and we must subtract those separately.

In $12 \%$ of the signal events, we can identify an electron or muon from the decay of the other $B$ [31](lepton tagging). After lepton tagging, the remaining background sample mainly comes from other $B$ decays (and is very suppressed). The reason why we look at the leptontagged sample, though clearly affected by having low statistics, is because it is a very clean sample.

For both lepton-tagged and untagged events, we give results in eight categories defined by: $M_{X s}=0.6-1.1 \mathrm{GeV}$ (essentially the $K^{*}$ resonance), $M_{X s}=1.1-1.5 \mathrm{GeV}, M_{X s}=1.5-1.9 \mathrm{GeV}$, $M_{X s}=1.9-2.3 \mathrm{GeV}, M_{X s}=0.6-2.3 \mathrm{GeV}$ (all events), $B^{0}$ decays with a $K^{ \pm}, B^{ \pm}$decays with a $K^{ \pm}$and $B^{ \pm}$decays with a $K_{S}$.

We separate the $B^{0}$ decays with a $K_{S}$ daughter for systematic studies. They are used in the study of the Monte Carlo modeling of the $X_{S}$ fragmentation. In principle, if there is a lepton-tag it can be used to tag $(C P)$ these events, but with a dilution due to $B^{0}-\bar{B}^{0}$ mixing. So, we do not include these modes in our direct $C P$ violation measurement.

Note that sometimes an iType number references the decay mode. The iType number is an integer that indicates the final state we are referring to. It is used for coding purposes. Table 5 defines the mapping between the iType number and the final state. The mapping does not depend on the $b$ flavor. The two $b$ flavors are also explicitly indicated in the table for clarity. 


\begin{tabular}{|c|c|c|c|}
\hline \multirow[t]{2}{*}{ iType \# } & \multicolumn{3}{|c|}{ Final State } \\
\hline & $\bar{b}$ decay & $b$ decay & mixed-flavor \\
\hline 1 & $B^{+} \rightarrow K_{S} \pi^{+} \gamma$ & $B^{-} \rightarrow K_{S} \pi^{-} \gamma$ & \\
\hline 2 & $B^{+} \rightarrow K^{+} \pi^{0} \gamma$ & $B^{-} \rightarrow K^{-} \pi^{0} \gamma$ & \\
\hline 3 & $B^{0} \rightarrow K^{+} \pi^{-} \gamma$ & $\bar{B}^{0} \rightarrow K^{-} \pi^{+} \gamma$ & \\
\hline 4 & & & $B^{0} \rightarrow K_{S} \pi^{0} \gamma$ \\
\hline 5 & $B^{+} \rightarrow K^{+} \pi^{-} \pi^{+} \gamma$ & $B^{-} \rightarrow K^{-} \pi^{-} \pi^{+} \gamma$ & \\
\hline 6 & $B^{+} \rightarrow K_{S} \pi^{+} \pi^{0} \gamma$ & $B^{-} \rightarrow K_{S} \pi^{-} \pi^{0} \gamma$ & \\
\hline 7 & $B^{+} \rightarrow K^{+} \pi^{0} \pi^{0} \gamma$ & $B^{-} \rightarrow K^{-} \pi^{0} \pi^{0} \gamma$ & \\
\hline 8 & & & $B^{0} \rightarrow K_{S} \pi^{+} \pi^{-} \gamma$ \\
\hline 9 & $B^{0} \rightarrow K^{+} \pi^{-} \pi^{0} \gamma$ & $\bar{B}^{0} \rightarrow K^{-} \pi^{+} \pi^{0} \gamma$ & \\
\hline 10 & & & $B^{0} \rightarrow K_{S} \pi^{0} \pi^{0} \gamma$ \\
\hline 11 & $B^{+} \rightarrow K_{S} \pi^{+} \pi^{-} \pi^{+} \gamma$ & $B^{-} \rightarrow K^{-} \pi^{-} \pi^{+} \pi^{0} \gamma$ & \\
\hline 12 & $B^{+} \rightarrow K^{+} \pi^{-} \pi^{+} \pi^{0} \gamma$ & $B^{-} \rightarrow K^{-} \pi^{-} \pi^{+} \pi^{0} \gamma$ & \\
\hline 13 & $B^{+} \rightarrow K_{S} \pi^{+} \pi^{0} \pi^{0} \gamma$ & $B^{-} \rightarrow K_{S} \pi^{-} \pi^{0} \pi^{0} \gamma$ & \\
\hline 14 & $B^{0} \rightarrow K^{+} \pi^{-} \pi^{+} \pi^{-} \gamma$ & $\bar{B}^{0} \rightarrow K^{-} \pi^{+} \pi^{+} \pi^{-} \gamma$ & \\
\hline 15 & & & $B^{0} \rightarrow K_{S} \pi^{0} \pi^{+} \pi^{-} \gamma$ \\
\hline 16 & $B^{0} \rightarrow K^{+} \pi^{-} \pi^{0} \pi^{0} \gamma$ & $\bar{B}^{0} \rightarrow K^{-} \pi^{+} \pi^{0} \pi^{0} \gamma$ & \\
\hline
\end{tabular}

Table 5: Reconstructed decay modes, corresponding coding referred to by "iType", and distinction among $\bar{b}$ and $b$ final states. The last is important for our definition of the $A_{C P}$. The mixed-flavor states are only used for systematic studies. 


\section{Selection}

We're not going to need the $D_{S}^{*}$, are we?

Our direct $C P$ violation measurement is statistics limited. Most systematic errors cancel in the asymmetry ratio of two $C P$ states. In particular, the signal and background shapes, the spectral shape, and the fragmentation of the $X_{S}$ system are expected to be the same. There are small differences in reconstruction efficiency and particle identification which are discussed in the section on systematics.

This makes the optimization of selection cuts straightforward. The statistical error on the asymmetry is minimized if we maximize the ratio $S^{2} / S+B$, normalized to the expected luminosity or number of signal and background events. The background is the sum of the continuum and $B \bar{B}$ contributions, except when otherwise specified.

The selection criteria that we apply in the analysis can be summarized in several steps:

- Initial Reconstruction: First we select events according to the event shape and signal photon cuts. We then reconstruct the $B$ decaying into an energetic photon, a kaon and $n$ pions in the final states. The total number of reconstructed final states for the analysis is 16 , corresponding to $n \leq 3$ (we only use 12 of the 16 modes for the $C P$ measurement, as already mentioned).

- Particle Quality Criteria: We apply quality cuts on the photons, kaons and pions which belong to the reconstructed $B$ candidates.

- Neural Network: We then construct a neural net variable, by means of shape variables.

- Selection: We cut on the neural network distribution and on other variables. 
- Tagging: We split the events in "lepton-tagged" and "untagged" samples (The leptontagged sample is a sub-sample of the untagged sample). The lepton-untagged sample is made of all the events that pass the selection, while the lepton-tagged sample is made only of the events whose "other" $B$ is lepton tagged.

- Multiple Candidates choice: After this selection there is usually more than one $B$ candidate left in each event. We then choose the best candidate per event, taking the one with the minimum $\Delta E^{*}$.

Each step is described in detail in the following sections.

\subsection{Initial Reconstruction}

We start with all events and then apply the same cuts listed below:

- The second Fox-Wolfram moment, R2<0.9² [32].

- At least one photon with energy, $E_{\gamma}^{*}$ in the interval $1.5 \mathrm{GeV}<E_{\gamma}^{*}<3.5 \mathrm{GeV}$ in the CMS frame.

- At least three tracks from the pion list. The quality cuts applied on the tracks, according to the pion selection, are shown in Table 6 .

We then reconstruct the final states described in Table 5 applying three more loose cuts on the reconstructed $B$ candidates to reduce the multiplicity:

- $\left|\Delta E^{*}\right|<0.4 \mathrm{GeV}$.

\footnotetext{
${ }^{2}$ For our analysis the Fox-Wolfram moments are defined by:

$$
H_{l}=\sum_{i} \frac{\left|p_{\gamma}\right|\left|p_{i}\right|}{E_{v i s i b l e}^{2}} P_{l}\left(\cos \left(\theta_{i}\right)\right)
$$

where $P_{l}(x)$ are the Legendre polynomials, $\theta_{i}$ is the opening angle between the photon and hadrons, and $p_{i}$ is the momentum of the hadrons.
} 


\begin{tabular}{|l|l|}
\hline Variable & Cut \\
\hline Minimum Transverse momentum & $0.1 \mathrm{GeV}$ \\
Maximum momentum & $10 \mathrm{GeV}$ \\
Minimum number of DCH hits & 12 \\
Minimum fit $\chi^{2}$ probability & 0 \\
Maximum DOCA in XY plane & $1.5 \mathrm{~cm}$ \\
Minimum Z DOCA & $-10 \mathrm{~cm}$ \\
Maximum Z DOCA & $10 \mathrm{~cm}$ \\
\hline
\end{tabular}

Table 6: Cuts applied by the pion selection. "DOCA" is the distance of closest approach to the beam spot.

- $m_{\mathrm{ES}}>5.2 \mathrm{GeV}$.

- $5.13<m_{B}<5.43 \mathrm{GeV}$.

Where $m_{B}$ is the $B$ meson mass reconstructed from the $B$ daughters.

The efficiencies, after all initial cuts are applied and the 12 final states are reconstructed, are shown in Table 7 . The corresponding average number of candidates after cuts and reconstruction, assuming at least one candidate reconstructed, are shown in Table 8.

\begin{tabular}{|l|c|}
\hline Sample & efficiency in \% \\
\hline$b \rightarrow s \gamma$ signal & 32.2 \\
$c \bar{c}$ & 2.1 \\
$u d s$ & 2.4 \\
generic $B \bar{B}$ & 0.4 \\
\hline
\end{tabular}

Table 7: Efficiencies obtained from MC signal and background events after all initial cuts.

\subsection{Particle Quality Criteria}

After initial reconstruction of the $B$ candidates, we apply quality cuts on the reconstructed $B$ decay daughters. But before we apply individual particle cuts, we cut on the absolute value of the cosine of the angle $\theta_{T^{*}} .\left|\cos \theta_{T^{*}}\right|$ is the angle between the reconstructed $B$ daughters 


\begin{tabular}{|l|c|}
\hline Sample & multiplicity \\
\hline$b \rightarrow s \gamma$ signal & 6.8 \\
$c \bar{c}$ & 6.7 \\
$u d s$ & 5.6 \\
generic $B \bar{B}$ & 11.1 \\
\hline
\end{tabular}

Table 8: Average number of candidate multiplicities obtained from MC signal and background events after all initial cuts. At least one candidate is required.

and the rest of the reconstructed particles in the lab frame. The corresponding distribution can be seen in Figure 15. $\left|\cos \theta_{T^{*}}\right|$ is flat for signal, but rapidly increases at \pm 1 for the continuum background due to its jet-like structure. The cut we apply is 0.8 (optimized against the continuum background only). We then apply quality cuts on the individual $B$ daughters.

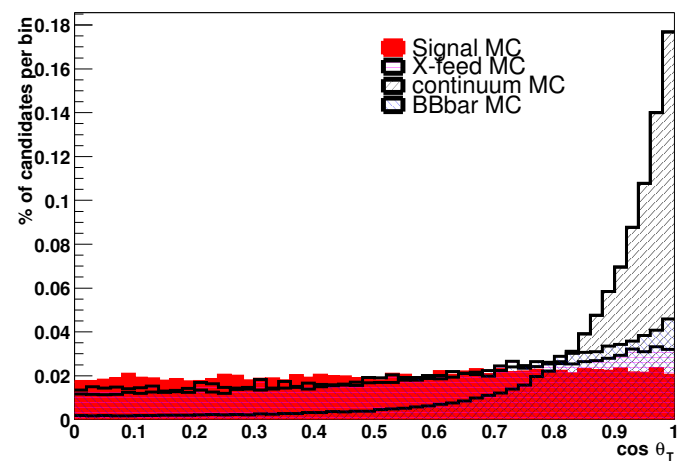

Figure 15: The $\left|\cos \theta_{T^{*}}\right|$ distribution. The histograms are all normalized to an area of 1 .

\subsubsection{High Energy $\gamma$ Selection}

High Energy Gamma List For use in this analysis, we provide a specially defined high energy photon list which selects photons according to a user-defined energy window. The 
selector takes our photon list ${ }^{3}$ as input, then applies a cut on $E_{\gamma}^{*}$, and returns a high energy photon list. We require that the photon associated to the reconstructed B-meson has an energy of at least $1.8 \mathrm{GeV}$. This cut reduces combinatorics without affecting the signal.

Photon Quality Cuts We then implement quality cuts on the photon selection (not already implicit in the choice of the photon list):

- The cluster does not contain a noisy or dead crystal.

- The cluster Second Moment <0.002.

- The photon theta angle in the LAB frame, $\theta_{\gamma}$, is in the fiducial interval: $-0.74<$ $\cos \theta_{\gamma}<0.93$.

The Second Moment is a measure of how circular the shower in the calorimeter is, and a cut on this reduces background from merged $\pi^{0}$ 's or $\eta$ 's. If the two photons from a $\pi^{0}$ or $\eta$ decay are merged and form a cluster, this cluster is more likely to be oval shaped. Clusters from one photon should be spherical which gives a small second moment.

Bump Distance Cut In order to improve the suppression of background, we implement an isolation cut on the photon bump (see section 3.2.4): the distance between the photon bump and another neutral bump or track in the event is computed. The distance between the photon and any other neutral bump or track on the event is asked to be greater than $20 \mathrm{~cm}$. This cut is done early in the analysis to reduce multiplicity and is tightened later on for more background suppression.

\footnotetext{
${ }^{3}$ The photon list is created from neutral calorimetric clusters which are single bumps (see section 3.2.4) not matched with any tracks, which have a minimum raw energy of $30 \mathrm{MeV}$, with a lateral moment $\leq 0.8$. The lateral moment is a measure of the spread of the cluster in the EMC. Our signal photons should be not spread out across the calorimeter, thus they should have a small lateral moment.
} 


\subsection{2 $\pi^{ \pm}$and $K^{ \pm}$Selection}

Tracks are requested to pass the pion selection, shown in Table 6. We do not apply particle identification (PID) to charged pions. We do require PID for charged kaons. We chose a tight selection as it has a high rejection power and the loss in signal efficiency is acceptable. The choice of tight kaon particle identification also cuts down on the raw number of combinatoric background in signal MC. Requiring PID for the kaon helps to suppress the small expected background from $b \rightarrow d \gamma$. The corresponding mis-id rate for pions as charged kaons is about $2 \%$ for our kaon selector.

\subsection{3 $K_{S}$ Selection}

Our reconstructed $K_{S}$ 's comes from the identification of the $K_{S} \rightarrow \pi^{+} \pi^{-}$decays. The $K_{S}$ 's are reconstructed from all pairs of oppositely charged tracks which are assigned the $\pi$ mass. The reconstructed $K_{S}$ mass must be within $15 \mathrm{MeV}$ of the nominal mass. For the $K_{S} \rightarrow \pi^{+} \pi^{-}$channels we currently use the cuts:

- A mass cut $489 \mathrm{MeV}<M_{K_{S}}<507 \mathrm{MeV}$ (see Figure 16 for MC mass fits to truth matched signal events).

- A decay length cut $>2.0 \mathrm{~mm}$ (see Figure 16 for the flight distributions for $K_{S}$ signal events, continuum, and generic $B \bar{B}$ background).

- Convergence of the vertex fit. An explicit requirement on the vertex fit is needed as no convergence is actually required in the $K_{S}$ list, and, if the vertexing does not converge, 4-vector addition is used. 

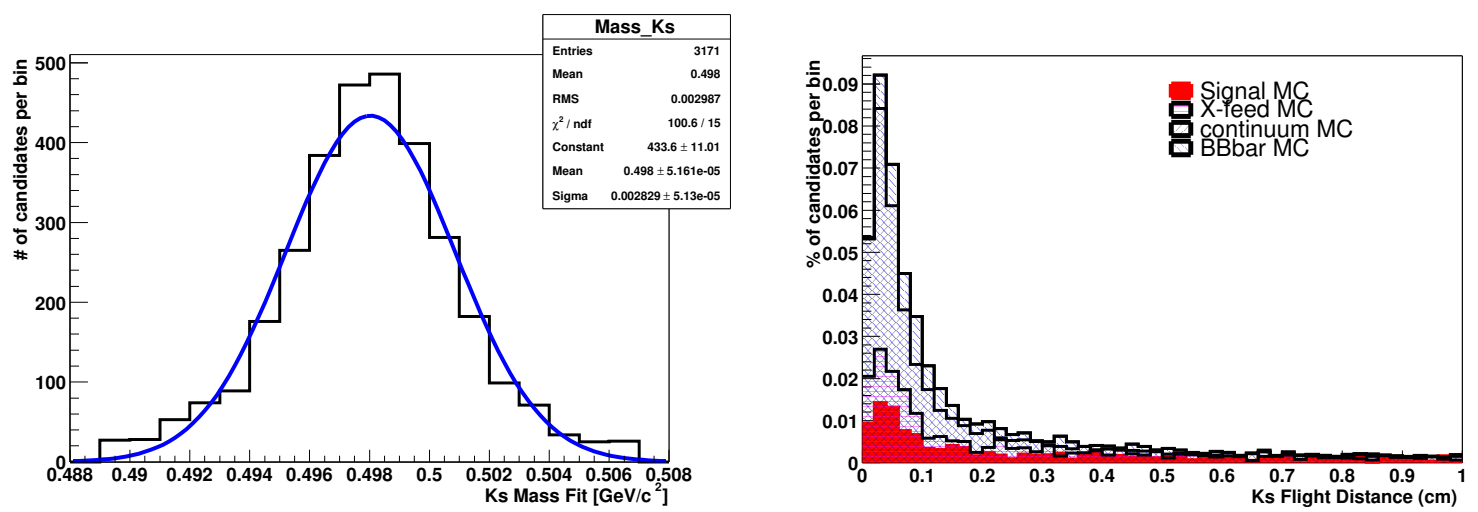

Figure 16: MC mass fits to truth matched signal and flight distributions for $K_{S}$ signal events, continuum, and generic $B \bar{B}$ background. The histograms are all normalized to an area of 1 , so their shapes can be compared but not their relative heights. $K_{S}$ flight distance plot normalized to one.

\subsection{4 $\pi^{0}$ Selection}

Our neutral pion candidates uses the reconstruction of $\pi^{0} \rightarrow \gamma \gamma$ for our selection criteria. The neutral pions are comprised of photons that belong to the photon list. At the initial reconstruction level the momentum of the $\pi^{0}$ is required to be at least $200 \mathrm{MeV}$. The raw mass is constrained to be in the window $90-170 \mathrm{MeV}$ (see Figure 17). A mass constrained fit is then applied.

\subsection{Neural Network (NN)}

Most of the background in the inclusive analysis arises from continuum production of a high energy photon either through initial state radiation (ISR) or through a $\pi^{0} / \eta$ decay. These backgrounds are suppressed using event shape variables. The shape variables are described in the following. However, instead of applying a cut for each variable separately, they are combined in a neural net in order to improve the total rejection power.

The neural net is a standard supervised learning, feedforward, backpropagation, neural 


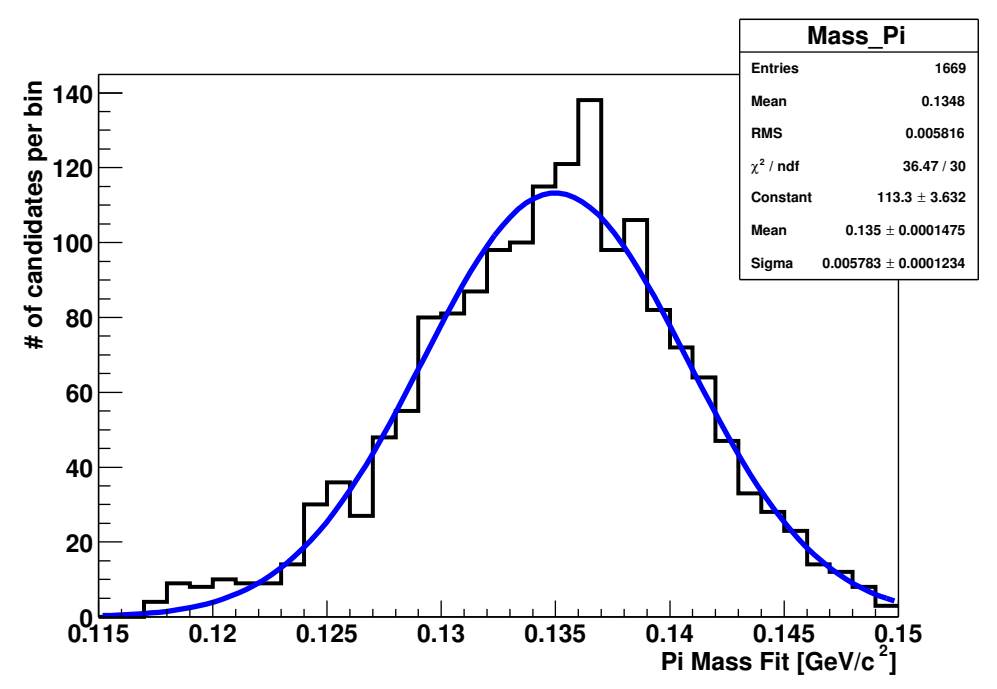

Figure 17: MC signal mass fits for truth matched $\pi^{0}$ 's.

network [33]. We use the Stuttgart Neural Network Simulator [34], with a developed Root based front end called RooCards ${ }^{4}$. We use 25 input variables, one hidden layer with 50 hidden nodes, and one output node to differentiate signal from continuum background. The details and validation are discussed in the next few sections.

\subsubsection{Input Variables for the Neural Net}

The shape variables that are used as input to our neural net are:

$\cos ($ thrust $)$ : The cosine of the reconstructed $B$ direction with respect to the $\mathrm{z}$ axis. For continuum this is peaked towards one, while for signal it is lower.

$\left|\cos \theta_{T^{*}}\right|$ : Although a loose cut at 0.8 is already applied on this variable, we find effective to include it in the neural net, as it still has a good separation between signal and background events.

$\left|\cos \theta_{B^{*}}\right|$ : The angle of the reconstructed $B$ candidate with respect to the beam direction

\footnotetext{
${ }^{4}$ RooCards is a privately developed interface between ROOT and SNNS.
} 
computed in the CMS frame. For signal events, $\left|\cos \theta_{B^{*}}\right|$ should fall off at high values; for background, $\left|\cos \theta_{B^{*}}\right|$ should be flat given the direction of the $B$.

$\left|\cos \theta^{\prime}\right|$ : Cosine of the photon in the recoil (or primed) frame. It is defined from the beam momenta and the reconstructed high-energy photon candidate by $\vec{p}\left(e^{+}\right)+\vec{p}\left(e^{-}\right)-\vec{p}(\gamma)=$ 0 . This is therefore the $q \bar{q}$ rest-frame for an ISR event; in it, the ISR background has a two-jets structure.

W2 : The second Fox-Wolfram moment.

$R 2$ : The ratio of second to zeroth Fox-Wolfram moment. A lower R2 is characteristic of the isotropic decay of signal events, as opposed to Bhabha and tau events and continuum background.

$R 2^{\prime}$ : R2 computed in the photon recoil system (defined above). This is useful in further reducing continuum events with initial state radiation. By investigating the frame without the potential ISR photon, the $R 2^{\prime}$ value can filter out continuum.

$E_{\theta}(1-18)$ : Energy flow cones in the CMS frame about the reconstructed $B$ direction, in $10^{\circ}$ increments (18 total). By looking at the energy flow cones we can separate out continuum from the more isotropic/spherical signal events. The energy of the reconstructed side of the event is removed from the cones.

Plots of the six shape variables can be found in Figure 18, the $\left|\cos \theta_{T^{*}}\right|$ variable can be found in Figure 15, normalized to the same area since relative shape is the important factor.

\subsubsection{Neural Network Basics}

A neural network is used to combine our twenty-five variables (see Section 6.3.1) into a single variable to separate the signal and background processes. The basic structure of a neural 

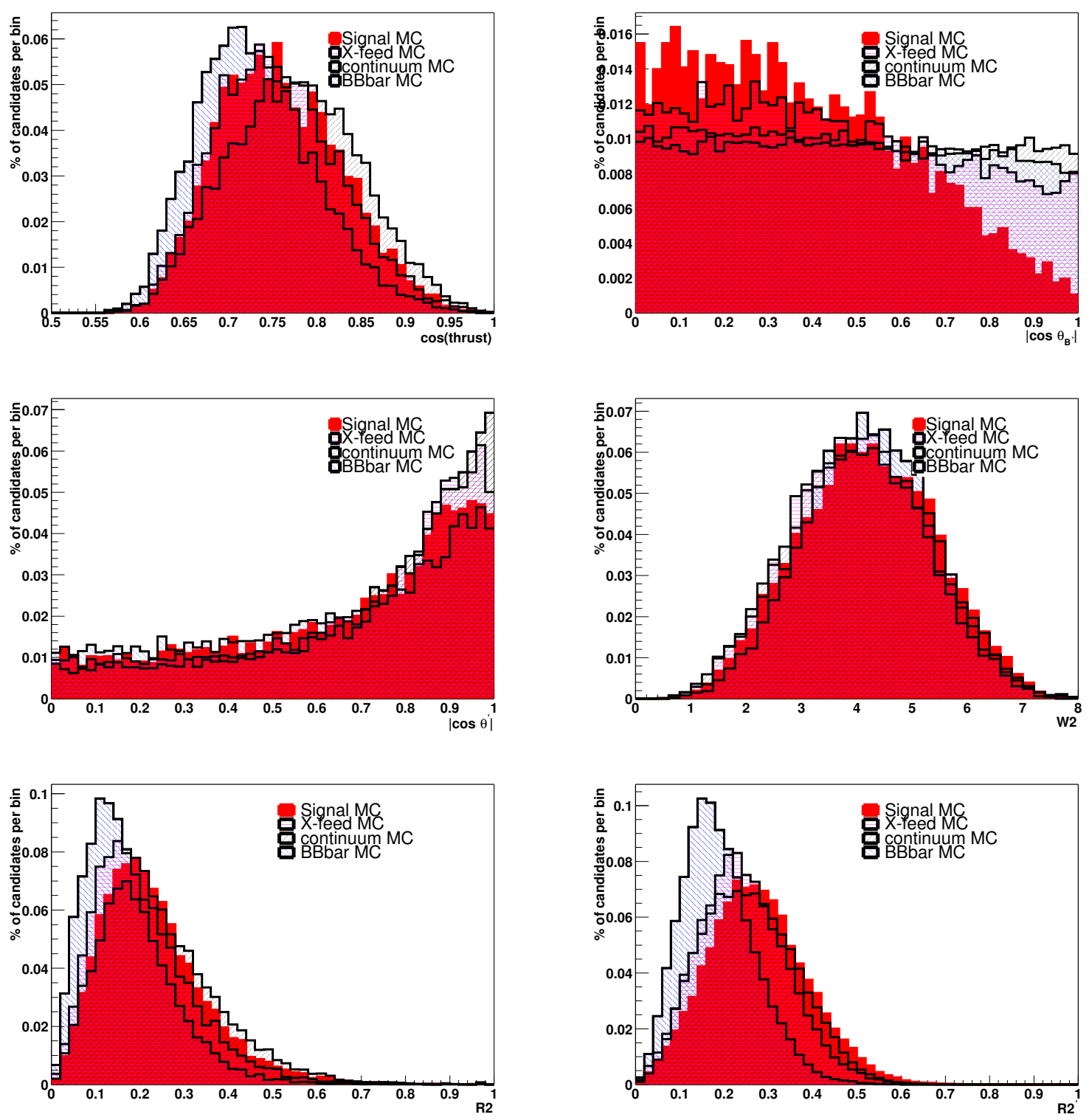

Figure 18: Shape variables going into the neural net: $\mid \cos ($ thrust $),\left|\cos \theta_{B^{*}}\right|,\left|\cos \theta^{\prime}\right|, W 2$, $R 2$ and $R 2^{\prime}$. The histograms are all normalized to an area of 1 . 
network is depicted in Figure 19. At the bottom of the diagram, event variables $x_{i}$ enter the network as input nodes in red. Linear combinations of these variables are sent up to the hidden nodes (blue): the $j$ th hidden node will receive receive the linear combination:

$$
y_{j}=\sum a_{i j} x_{j}
$$

Each hidden node receives a different linear combination of the input variables. This input is then transformed by an "activation" function, in this case the tanh function (our $x_{j}$ and $g\left(y_{j}\right)$ functions). The activation function determines how fast the output of the hidden layer varies as a function of the input. If the input range is small, a linear response is recovered; if it is large, a step response results. At each stage, there is "bias" node (purple) which provides a constant output. The bias node is used to set the zero-level of the output.

A linear combination of the output from the hidden nodes $g\left(y_{j}\right)$ :

$$
z=\sum b_{j} g\left(y_{j}\right)
$$

are then passed to the output node where it is again transformed by an activation function. The mathematical chain from input to output is shown on the right of Figure 19. A neural network can in principle have many hidden layers and output nodes. In general, neural network structures can be specified by the number of input nodes (network variables), the

number of hidden layers with the number of nodes in each hidden layer, and number of output nodes. Hence the example in Figure 19 can be described as a neural network with four input nodes, one hidden layer with three nodes and a bias node, and one output node. In this analysis, we end up using a configuration with one hidden layer.

\subsubsection{Training the Neural Network}

The free parameters of a neural network are the coefficients $a_{i j}$ and $b_{j}$ that are used to form the linear combination of input nodes to the hidden nodes, and the hidden node outputs to 


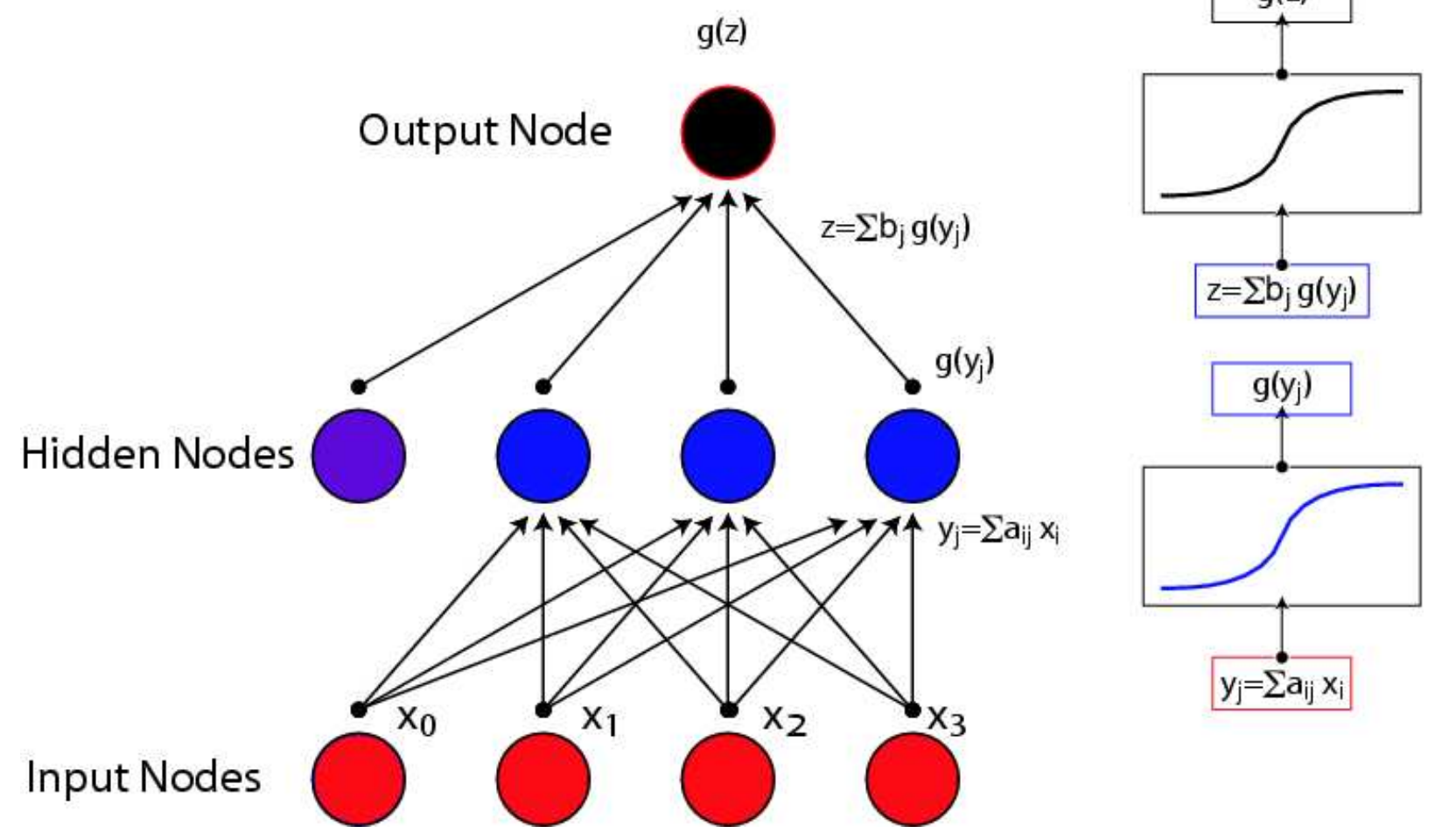

Figure 19: Basic structure of a single hidden layer neural network. Input nodes (red) corresponding to event variables are passed to "hidden" nodes (blue) as linear combinations. The line combination is then transformed bay an "activation function," in this case the $\tanh (x)$. Linear combinations of the hidden node outputs are then passed to the output node (black), where it is transformed once again by the activation function to give the final neural network output.

the output node respectively. The optimal set of coefficients are determined by a process known as "back-propagation." The performance of the neural network for any given set of coefficients is summarized by the sum-squared error (SSE):

$$
\operatorname{SSE}\left(a_{i j}, b_{j}\right)=\sum_{a=1}^{N}\left[\operatorname{NN}\left(\overrightarrow{x_{a}} ; a_{i j}, b_{j}\right)-F\left(\overrightarrow{x_{a}}\right)\right]^{2} .
$$

Here, the $x_{a}$ represents the vector of input variables for the $a$ th event, $\operatorname{NN}\left(\overrightarrow{x_{a}} ; a_{i j}, b_{k}\right)$ is the neural network output for this vector with the coefficients $a_{i j}$ and $b_{j}$, and $F\left(\overrightarrow{x_{a}}\right)$ is the desired output for this vector (e.g. 0 if it is a vector corresponding to a background event, 
1 if it is a signal event). The SSE then represents a " $\chi$ " for the network configuration that can be minimized in a manner completely analogous to a fit via gradient descent. This is precisely the back-propagation algorithm: the derivatives of the SSE relative to changes in each of the coefficients are evaluated and the coefficients adjusted accordingly and iteratively to minimize the SSE.

\subsubsection{Note on Neural Networks}

Another way you can think of a neural net, is that it is a simple set of transformations. Starting with a vector $\mathrm{X}$, we apply a transformation matrix and get to the next layer in our neural net. Using our neural net in Figure 19, we can see that our initial input nodes would be our vector $\mathrm{X}$, and we have two transformations to get to our output vector (node). The values of the transformation matrices are set from the training procedure. All matrix elements are initially randomized, and then using a procedure of successive approximations we set the individual matrix element values.

It is then easy to see why training has to be well monitored. Too little training will give you a result, but not the best result. Too much training (or not a large enough training set) can cause the transformation matrices to learn about your specific training set.

\subsubsection{Training/Optimization of the Neural Net}

The training samples used in the neural net were created from a reduced data set using all previously discussed cuts in Section 6.2.

We use truth-matched signal events (as described in section 4) for signal and $u d s$ and $c \bar{c}$ MC for background ${ }^{5}$. If there are more $B$ candidates per event, the one with the smallest $\Delta E^{*}$ is chosen.

\footnotetext{
${ }^{5}$ Generic $B \bar{B}$ MC was not used as a background-training sample. The generic $B \bar{B}$ sample introduces biases toward acceptances of lower multiplicity modes if used.
} 
We find that a ratio of $7 / 8$, signal to background is the optimal ratio of input events for training the neural net. Total data sets of 1400 signal and 1600 background events are used for the final training sets (no improvements are seen with larger data sets).

One hidden layer with 50 hidden nodes is the best configuration for our neural net. An increase or decrease in the number of hidden nodes actually shows a loss of signal/background differentiation. Using 50 hidden nodes in more than one hidden layer shows no improvement in signal or background definition.

Training is done in a round-robin format. Signal and background samples are broken up into three sub-samples. Two of these samples are used for training, and the third for testing. For each of the three training/validation sets a best number of training cycles is found along with a best neural net cut point. An example can be found in Table 9 .

\begin{tabular}{|c|c|c|}
\hline Sample & \# Cycles & Best NN Cut Point \\
\hline Training 1 & 400 & 0.62 \\
Training 2 & 400 & 0.66 \\
Validation & 350 & 0.62 \\
\hline
\end{tabular}

Table 9: For a neural round-robin training set, the optimum number of cycles to train per sub-sample, and the best neural network cut point.

The neural network output is shown in Figure 20 for various MC samples. Each candidate has a neural network computed weight; 1 is signal like while 0 is continuum background like.

\subsubsection{Comparison to a Linear Discriminant}

In a previous version of this analysis, performed with only a quarter of the data, a Fisher (linear) Discriminant was used for continuum background suppression. We check if we have gained any improvement in $S^{2} / S+B$ using the neural net instead of the Fisher Discriminant. Current neural net signal and background efficiencies are $73.9 \%$ and $31.4 \%$ respectively, relative just to the particle quality criteria cuts, while for the old Fisher Discriminant the 
signal and background efficiencies were $20.4 \%$ and $2.7 \%$, respectively. Using the expected yields, we have an $S^{2} / S+B=47.0$ for the new neural net, while the old Fisher Discriminate had an $S^{2} / S+B=36.6$. Thus, we are able to allow more than three times more signal through, while still improving our $S^{2} / S+B$ by $28 \%$.

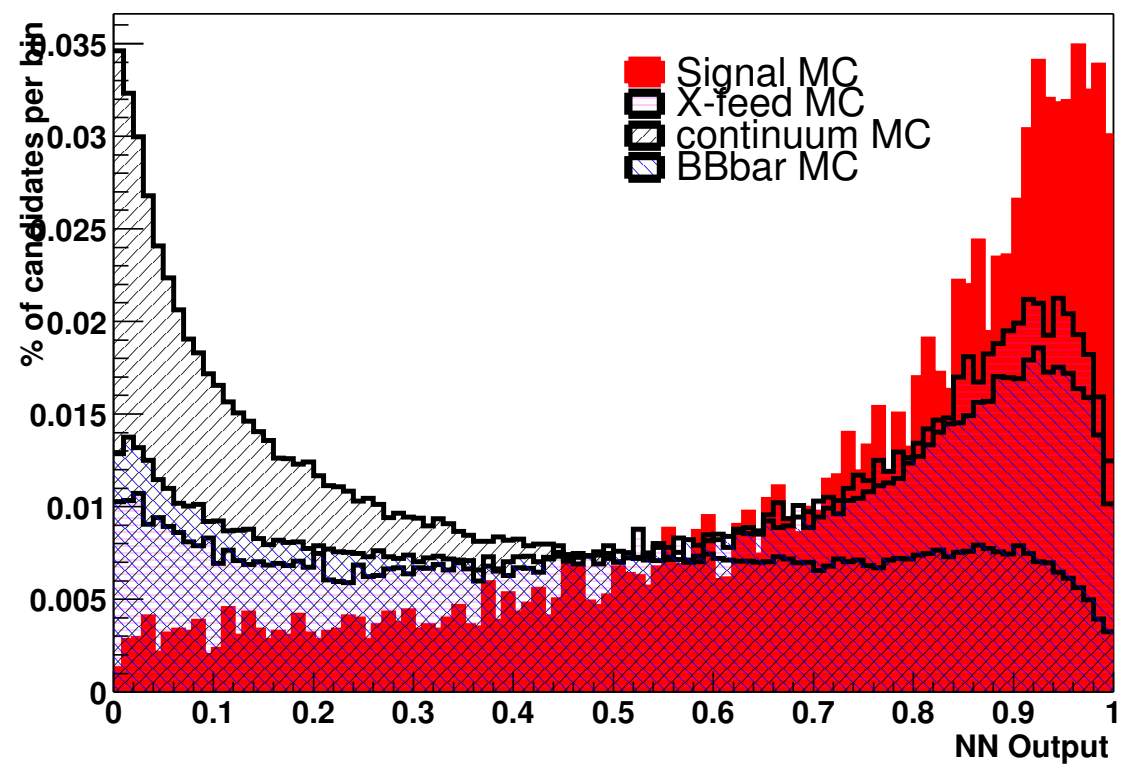

Figure 20: Neural net output for various MC samples: 1 is signal like, while 0 is continuum background like. The histograms are all normalized to an area of 1 .

\subsection{Final Selection Cuts}

Using the neural net and a few more discriminating variables, we make our final selection cuts. The criteria used to optimize the cuts is $S^{2} / S+B$. The background is the sum of continuum and $B \bar{B}$ events except for the optimization of the neural net and the minimum $K$ and $\pi$ momentum cuts, which are devised to get rid of continuum and $B \bar{B}$ events, respectively. A lower cut on $m_{\mathrm{ES}}$ at $5.27 \mathrm{GeV}$ is applied when computing $S^{2} / S+B$ as it corresponds to optimize the selection in the signal region. 
In the following, we describe the other variables used:

$\underline{\cos \theta_{B^{*}}}:$ Even though we have included $\cos \theta_{B^{*}}$ in the neural net (see section 6.3), we can still improve our $S^{2} / S+B$ by applying a harder cut on $\cos \theta_{B^{*}}$ by itself. We must remember that neural nets are good at multivariable relationships, but bad at single variable cutting. So, if we have a variable that by itself can provide a good cut, and the relationship between that variable and other variables can be used in a multivariable analysis, then the variable should be cut on and still used in the multivariable analysis (i.e. our neural net). Thus, we require that $\cos \theta_{B^{*}}<0.8$ besides the use of it in the neural net.

$\Delta E^{*}$ window: Random combinatoric background is reduced by putting tight constraints on our $\Delta E^{*}$ distribution. In Figure 21 we can see the $\Delta E^{*}$ distribution for signal and backgrounds. We cut at: $-0.1<\Delta E^{*}<0.1 \mathrm{GeV}$.

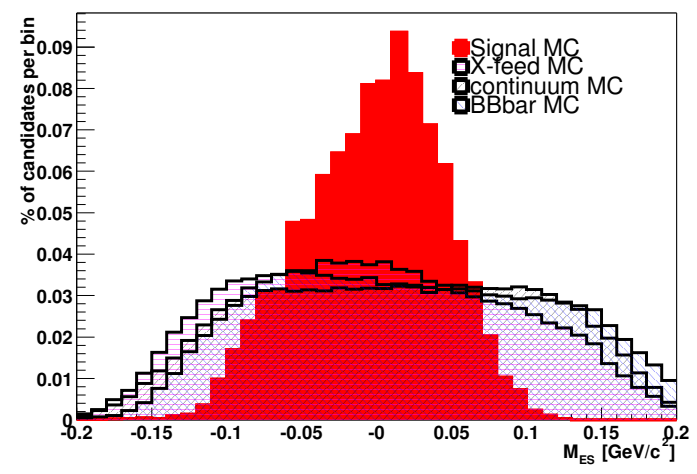

Figure 21: Plot of $\Delta E^{*}$ for signal and background. The falling off of the background $\Delta E^{*}$ distributions come from cuts made before this plot was made. The histograms are all normalized to an area of 1 .

Neural Network Cut: The minimum neural net output is requested to be 0.4 ( 1 is signal like while 0 is continuum background like). Note that in Table 9 the neural net cut is tighter 
than 0.4 because there were no cuts on other variables differently from the current case.

Minimum $K$ and $\pi$ Momentum: In order to reduce the combinatorial background from low momentum pions and kaons we set minimum momenta requirements. Since the higher multiplicity modes carry less momentum (see Figure 22) we must have a sliding scale. This cut also allows us to not worry about discrepancies between lower and higher momentum particle reconstruction. The corresponding cuts can be found in Table 10. We require no minimum daughter momentum in the $K^{*}$ region $\left(0.6 \mathrm{GeV}<M_{X s}<1.1 \mathrm{GeV}\right.$ ) due to no gain in $S^{2} / S+B$.

\begin{tabular}{|c|c|c|}
\hline Number of $\pi^{\prime} s$ in Final State & Minimum $K$ Momentum $(\mathrm{GeV})$ & Minimum $\pi$ Momentum $(\mathrm{GeV})$ \\
\hline 1 & 0.7 & 0.5 \\
2 & 0.7 & 0.3 \\
3 & 0.7 & 0.2 \\
\hline
\end{tabular}

Table 10: Minimum $\pi$ and $K$ momentum cut per number of $\pi^{\prime} s$ in tag or untagged state with $1.1<M_{X s}<2.3 \mathrm{GeV}$.

The pion momentum cut was done with a best $S^{2} / S+B$ requirement. The minimum kaon momentum is chosen to be greater than $0.7 \mathrm{GeV}$ due to the kaon selection. Above 0.7 $\mathrm{GeV}$ the kaon selection uses DIRC information and is a much cleaner sample.

Bump distance Cut: A tighter selection that the one already devised in Section 6.2 is applied, tightening to $28 \mathrm{~cm}$ and $40 \mathrm{~cm}$ the maximum distance between the signal photon bump and the closest track and neutral bump, respectively. The corresponding distribution can be seen in Figure 23.

$\underline{\pi^{0} \text { and } \eta \text { Vetoes: }}$ There is also a background component in which one photon from an asymmetric $\pi^{0}$ or $\eta$ decay can have sufficient energy to be considered as our high energy signal photon candidate. A $\pi^{0}$ and $\eta$ veto is implemented to remove this. 


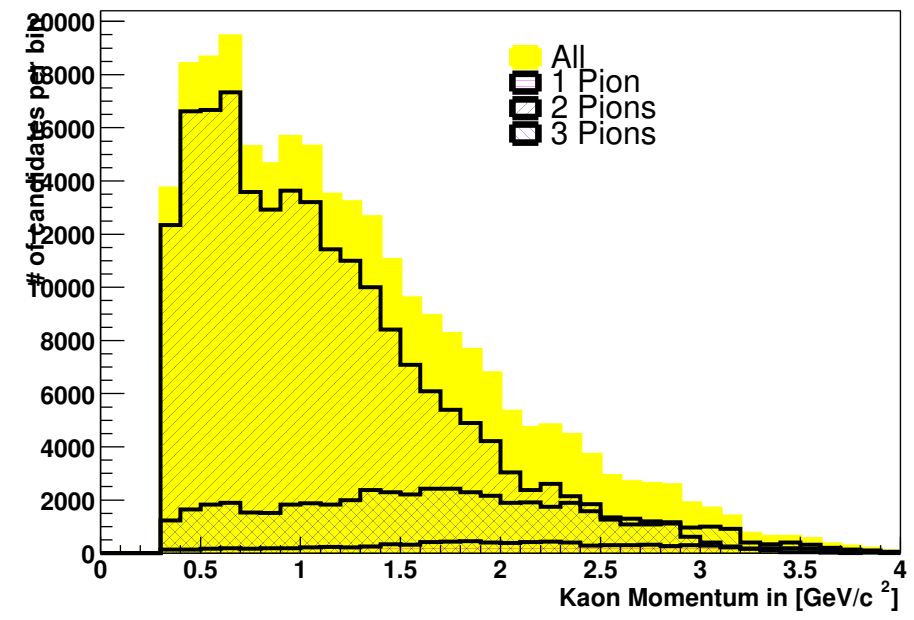

Figure 22: Kaon momentum for 2/3/4 body and all decays in our on-resonance sidebands. Notice the step like distribution before $0.7 \mathrm{GeV}$ due to no DIRC requirement for charged kaons with less than $0.7 \mathrm{GeV}$.
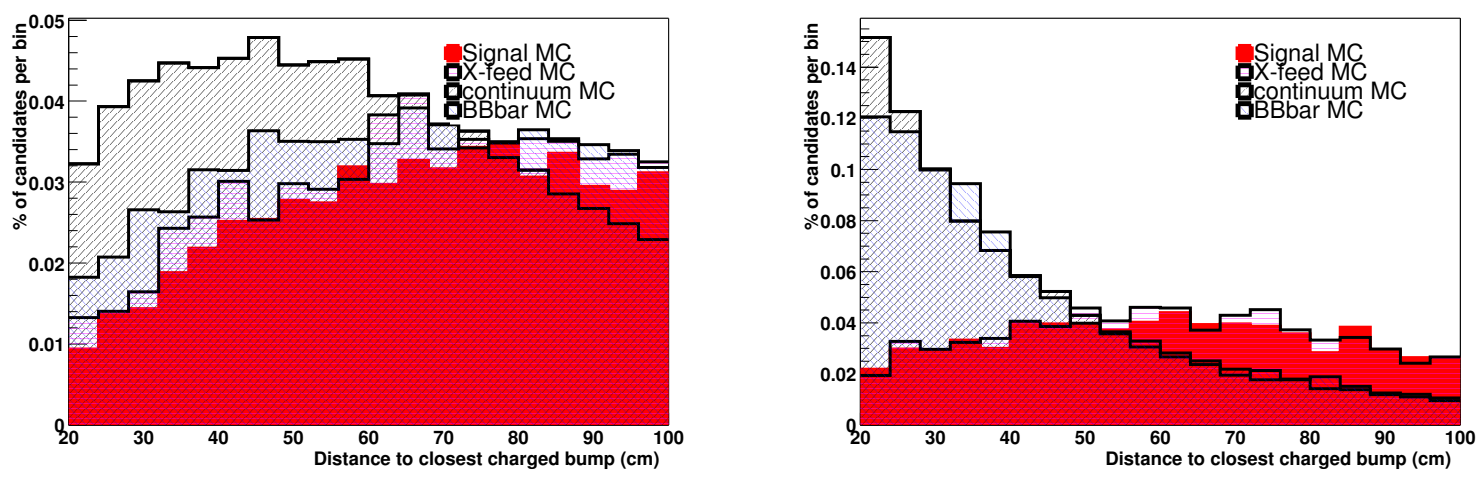

Figure 23: Charged(left) and neutral(right) bump distance distribution for signal and background MC events. The histograms are all normalized to an area of 1 . 
The "veto" $\pi^{0}$ 's are made of photons from the photon list and our high energy photon. The lower cut on the photon energy from the list is set to $50 \mathrm{MeV}$. The energy of the veto $\pi^{0}$ is required to be at least $200 \mathrm{MeV}$, and the veto $\pi^{0}$ 's raw mass must be in the window of $90-170 \mathrm{MeV}$. The "veto" $\eta$ 's are made of photons from the photon list and our high energy photon. The lower cut on the photon energy from the list is set to $100 \mathrm{MeV}$ and a cut on the $\eta$ mass between 470 and $620 \mathrm{MeV}$ is applied.

If there is a $\pi^{0}$ or $\eta$ veto candidate with a reconstructed mass in the range:

$$
\begin{gathered}
116 \mathrm{MeV}<m_{\pi^{0}}<152 \mathrm{MeV} \\
524 \mathrm{MeV}<m_{\eta}<566 \mathrm{MeV}
\end{gathered}
$$

the event is rejected. In Figure 24, the closest masses per event (in case of overlap) to the $\pi^{0}$ and $\eta$ PDG masses are shown for a sample of $X_{s} \gamma$ signal MC, cross-feed and generic $B \bar{B}$ background MC.
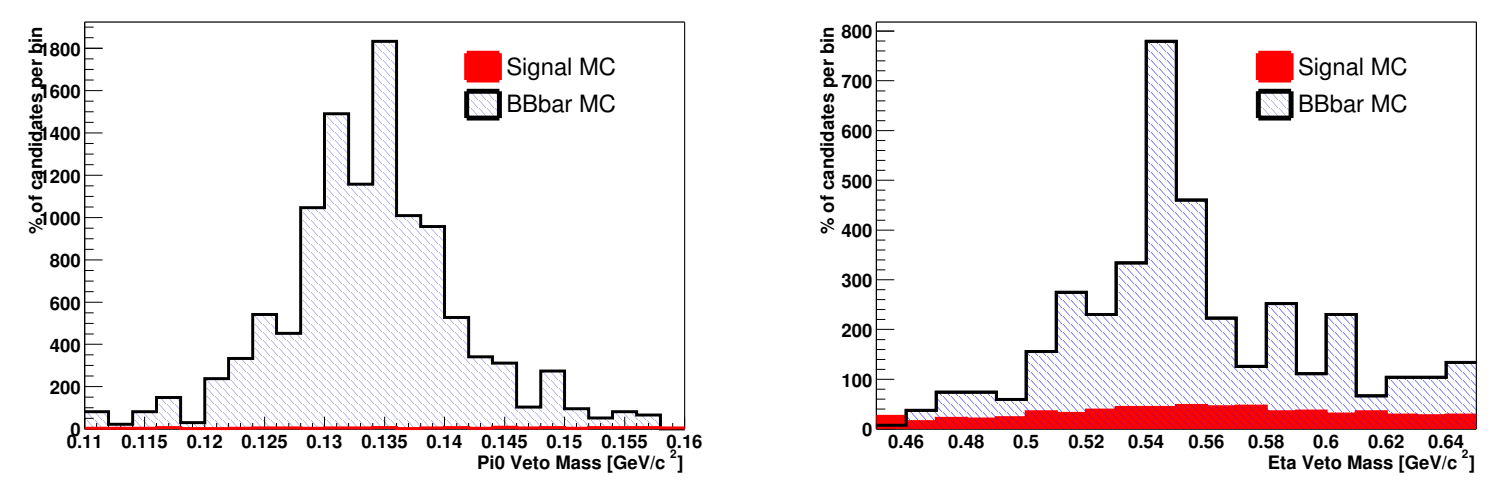

Figure 24: Closest masses per event (in case of overlap) to the $\pi^{0}$ (left) or $\eta$ (right) PDG masses for $X_{s} \gamma$ signal MC and generic $B \bar{B}$ background MC. The histograms are all normalized to the luminosity. 


\subsection{Tagging}

We break up the data sample into a "lepton-tagged" and "untagged" sample. The untagged sample is inclusive of all the events which pass the selection, while the lepton-tag sample is a sub-sample of the untagged one made of all the events which are lepton-tagged. We are currently using the Moriond Tagging method [31]. The Moriond Tagger uses all reconstructed tracks not used to produce our signal B-meson decay, and tries to determine if the reconstructed tracks could come from the other B-meson. The tracks are usually associated with high-energy leptons or $K$ mesons.

The tagged sample uses four Moriond Tagging categories: Electron/Kaon, Muon/Kaon, Electron only, and Muon only. The leptonic tag helps in reducing the continuum background for those events, which are tagged.

\subsection{Multiple Candidates Choice}

After all the candidate selection criteria are applied, on average we still have more than one candidate per event (see Table 11 for details). For the final fits, we only want one candidate per event. So, for best candidate selection, we choose the candidate with the smallest $\left|\Delta E^{*}\right|$.

\begin{tabular}{|l|c|}
\hline Sample & $\begin{array}{c}\text { Final average } \\
\text { multiplicity }\end{array}$ \\
\hline$b \rightarrow s \gamma$ signal (KN480) & 2.30 \\
$c \bar{c}$ & 2.29 \\
$u d s$ & 1.88 \\
generic $B \bar{B}$ & 1.96 \\
\hline
\end{tabular}

Table 11: Average candidate multiplicities obtained from MC after all cuts are made. 


\section{7 $\quad X_{S}$ Mass Cut-Off}

As referenced in Section 4, an asymmetry in $b \rightarrow s \gamma$ decays should not be dependent on the $X_{S}$ mass [25] (or signal photon energy). In order to find the best point to apply a cut-off on the hadronic mass, we need to apply all the selection criteria and finally fit the total expected number of signal and background events (the fit will be described in the next section). The criterion used to choose the best $M_{X s}$ cut-off is: the number of expected signal event, to the total statistical error of the peaking signal and background events. This is similar to

choosing the cut-off according to the usual $S^{2} / S+B$ criteria. However, minimizing the statistical error from the full fit takes into account the presence of a non-peaking component of our background (which is the dominant background contribution). A $M_{X s}$ mass cutoff of $2.3 \mathrm{GeV}$ is found to be optimal.

\begin{tabular}{||c|c|c|}
\hline$M_{X s} \mathrm{GeV}$ & Number of Signal & Number of Signal / Statistical Error \\
\hline $2.0-2.1$ & 79 & 1.86 \\
$2.1-2.2$ & 67 & 1.97 \\
$2.2-2.3$ & 51 & 1.88 \\
$2.3-2.4$ & 28 & 1.55 \\
\hline
\end{tabular}

Table 12: Number of signal events per $100 \mathrm{MeV}$ mass bin in $X_{S}$ (full $m_{\mathrm{ES}}$ range). The ratio of increased number of signal events to the statistical error in the peaking component of a fit to the signal background events is our criteria to choose the cut-off point.

\subsection{Final Efficiencies}

After the best candidate selection, we compare the final efficiencies and expected number of events for signal and background in Table 13. The corresponding breakdown of the efficiencies for the different selection criteria for the signal events can be found in Table 14 . 


\begin{tabular}{|l|c|c|}
\hline \multicolumn{3}{|c|}{ Signal MC category } \\
\hline Category & Efficiency $\%$ & Expected number of events \\
\hline$M_{X s}=0.6-1.1 \mathrm{GeV}$ & 17.1 & 784 \\
$M_{X s}=1.1-1.5 \mathrm{GeV}$ & 6.46 & 325 \\
$M_{X s}=1.5-1.9 \mathrm{GeV}$ & 3.73 & 190 \\
$M_{X s}=1.9-2.3 \mathrm{GeV}$ & 3.51 & 78 \\
$M_{X s}=0.6-2.3$ & 10.8 & 1394 \\
$B^{0}$ Decays & 11.4 & 786 \\
$B^{+}$Decays with a $K_{S}$ daughter & 10.4 & 250 \\
$B^{+}$Decays with a $K^{+}$daughter & 9.70 & 357 \\
\hline \hline \multicolumn{2}{|c|}{ Background } \\
\hline Background type & Efficiency $(\%)$ & Expected number of events \\
\hline Generic $B$ Background & 0.0014 & 1297 \\
Continuum Background & 0.0045 & 10036 \\
Cross-feed & - & 1210 \\
\hline
\end{tabular}

Table 13: Final efficiencies and expected number of events for different types of signal and background definitions. Expected number of events for full $m_{\mathrm{ES}}$ distribution.

\begin{tabular}{|c|c|c|c|}
\hline Cut & Signal \% & Generic B \% & Continuum \% \\
\hline$\left|\cos \theta_{T^{*}}\right|<0.8$ & 81.3 & 61.9 & 17.1 \\
$K_{S}$ Quality Criteria & 96.4 & 57.6 & 63.8 \\
Bump Distance $>20 \mathrm{~cm}$ & 100 & 81.7 & 79.0 \\
$\mathrm{E}_{\gamma}>1.8 \mathrm{GeV}$ & 99.2 & 30.2 & 49.2 \\
no noisy or dead crystal & 97.7 & 98.8 & 96.3 \\
cluster second moment $<0.002$ & 98.5 & 85.4 & 89.5 \\
$-0.74<\cos \theta_{\gamma}<0.93$ & 98.5 & 96.3 & 95.2 \\
\hline$\left|\cos \theta_{B^{*}}\right|<0.8$ & 91.6 & 86.5 & 79.8 \\
$-0.10<\Delta E^{*}<0.1 \mathrm{GeV}$ & 76.0 & 45.0 & 45.3 \\
NN Output $>0.4$ & 73.9 & 63.7 & 31.4 \\
Min $K$ and $\pi$ Momentum & 78.6 & 66.4 & 73.3 \\
Charged Bump Distance $>28 \mathrm{~cm}$ & 98.6 & 96.9 & 96.0 \\
Neutral Bump Distance $>40 \mathrm{~cm}$ & 87.2 & 50.0 & 60.6 \\
$\pi^{0}$ and $\eta$ Veto & 90.0 & 67.2 & 83.2 \\
\hline
\end{tabular}

Table 14: Efficiency of the cuts normalized to all cuts before (above) them for the $M_{X s}=1.1$ $2.3 \mathrm{GeV}$ mass range. 


\section{Fitting of Monte Carlo and Data}

It does not do to leave a live dragon out of your calculations, if you live near him.

We fit to the $m_{\mathrm{ES}}$ distribution since the expected signal and background shapes can be defined by well-known functions. The continuum background from $c \bar{c}$ and light quark jets can be fit with the Argus function [35] (see Appendix B for details on the fitting functions). The truth-matched (see Section 4) signal events can be fitted by a Crystal Ball function [36] to account for the radiative tail in the photon energy measurement (again see Appendix B for details). The number of events found by floating the normalization in the Crystal Ball function is our final extracted signal $(n(b \rightarrow s \gamma)$ and $n(\bar{b} \rightarrow \bar{s} \gamma))$.

The main challenge is to describe the shape of the generic $B \bar{B}$ and cross-feed backgrounds. Ideally we would define separate shape functions for these each data type. Due to the limited statistics of the current data sample, we need to reduce the number of degrees of freedom. All of the fitting procedures are done using Monte Carlo weighed to expected luminosities except where noted.

We can adequately describe both the generic $B \bar{B}$ sample and the cross-feed sample by the sum of an Argus and a Crystal Ball function. The background Crystal Ball shape is taken from the truth matched signal shape. We can also describe the sum of these two samples in the same way, i.e. by one Argus shape plus the signal Crystal Ball. Thus we fit the full sample with two Argus functions, and a Crystal Ball function. The Crystal Ball shape parameters are defined by a fit to the weighted average of the truth-matched signal Monte Carlo samples in hadronic mass bins. The Crystal Ball parameters show no dependence on the hadronic mass bin within our errors.

One Argus shape is fixed from a fit to the continuum Monte Carlo (see Figure 25). The second Argus shape is allowed to float to account for uncertainties in our knowledge of the 

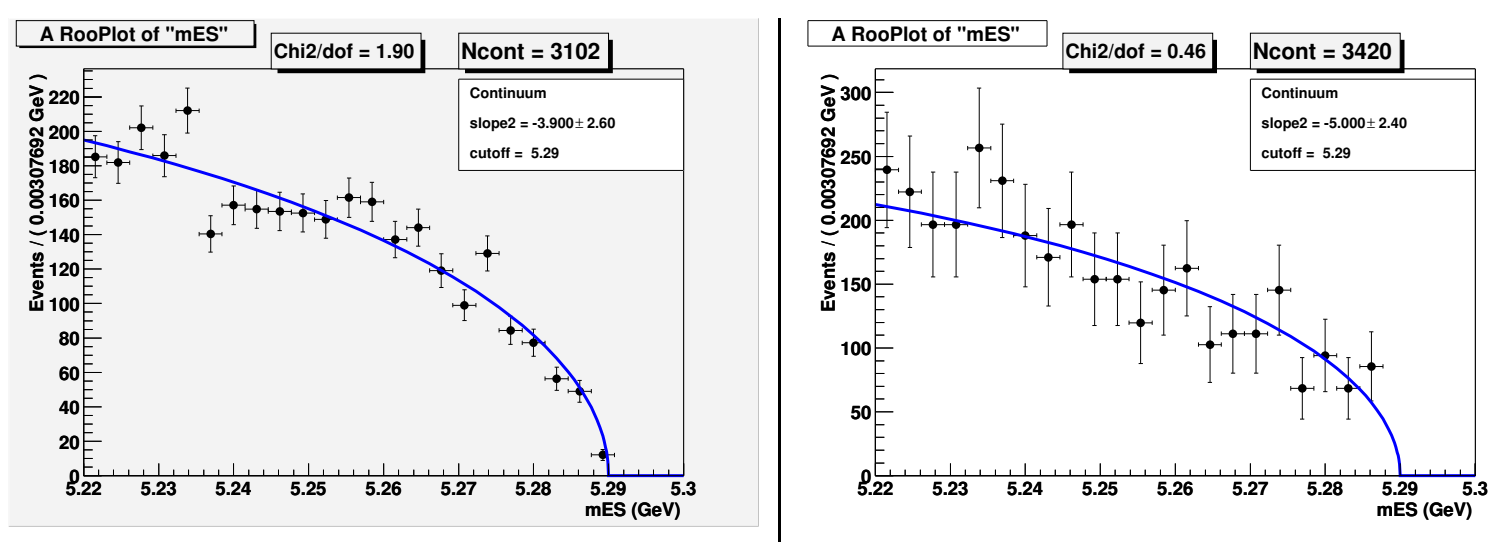

Figure 25: Plots of continuum Monte Carlo(left) and off-resonance data(right) for the 1.5$1.9 \mathrm{GeV}$ region. Both plots have be normalized to expected luminosity.

cross-feed and $B \bar{B}$ backgrounds. This gives us 3 degrees of freedom in our fits: the number of events in the signal Crystal Ball function, the relative number of events in the free Argus, and the slope parameter of the free Argus. We then subtract this small peaking background (cross-feed $+B \bar{B}$ Monte Carlo) from our data fits to find our signal. A summary of the functions used and parameters can be found in Table 15. A toy study was done to check if the fitting biased the results in any way, details of the study can be found in Appendix C.

From now on we will only be presenting numbers for $b$ events, $\bar{b}$ events, or the average of the two. A cross-check that the fitting parameters are consistent between $b$ and $\bar{b}$ events is made in Appendix D. We justify the fitting technique with toy Monte Carlo as shown in Appendix C.

\subsection{Fitting of the Different Monte Carlo Categories}

In the following, we describe the fits to our data sets according to the functions described in Table 15. 


\begin{tabular}{|c|c|}
\hline Data Set Type & $\begin{array}{c}\text { Fit Function } \\
\text { [free parameters] }\end{array}$ \\
\hline Signal MC & $\begin{array}{l}\text { Crystal Ball (free) } \\
{[\text { mean, } \alpha, \sigma, n]}\end{array}$ \\
\hline XFeed MC & Argus (free) + Crystal Ball (as signal) \\
\hline & [Argus shape parameter, relative \# of events in A and CB] \\
\hline$B \bar{B} \mathrm{MC}$ & Argus (free) + Crystal Ball (as signal) \\
\hline & [Argus shape parameter, relative \# of events in A and CB] \\
\hline Continuum MC & Argus (free) \\
\hline Off-resonance data & Argus (free) \\
\hline & [Argus shape parameter] \\
\hline $\mathrm{XFeed}+B \bar{B} \mathrm{MC}$ & $\begin{array}{c}\text { Argus (free) }+ \text { Crystal Ball (as signal) } \\
{[\text { Argus shape parameter, relative \# of events in A and CB }}\end{array}$ \\
\hline $\mathrm{XFeed}+B \bar{B}+$ Continuum $\mathrm{MC}$ & $\begin{array}{l}\text { Argus (free) }+ \text { Argus (as continuum) }+ \text { Crystal Ball (as signal) } \\
{[\text { Argus shape parameter, relative \# of events in A and A and CB] }}\end{array}$ \\
\hline $\begin{array}{l}\mathrm{XFeed}+B \bar{B}+\text { Continuum } \mathrm{MC} \\
+ \text { Signal MC }\end{array}$ & $\begin{array}{l}\text { Argus (free) }+ \text { Argus (as continuum) }+ \text { Crystal Ball (as signal) } \\
\text { [Argus shape parameter, relative \# of events in A and A and CB] }\end{array}$ \\
\hline Data & $\begin{array}{l}\text { Argus (free) }+ \text { Argus (as continuum) }+ \text { Crystal Ball (as signal) } \\
{[\text { Argus shape parameter, relative \# of events in A and A and CB }}\end{array}$ \\
\hline
\end{tabular}

Table 15: Functions and free parameters used to fit the various data samples. Legend: $\mathrm{A}=$ Argus function, $\mathrm{CB}=$ Crystal Ball function.

\subsubsection{Signal Fits}

In the top left plot of Figures 26 and 27 we show a typical $m_{\mathrm{ES}}$ signal shape for the region $M_{X s}=1.1-1.5 \mathrm{GeV}$. The Monte Carlo signal distribution, taken from either the flat signal Monte Carlo or the KN480 model, is weighted to match the expected data yields and then fit with a Crystal Ball function. The Crystal Ball shape parameters can be found in Table 16. Note that there is no apparent variation in these parameters as a function of $M_{X s}$, and we assume that the signal shape does not differ in $b$ and $\bar{b}$ events. When studying the systematic contributions in Section 9, we do check that this assumption is correct.

\subsubsection{Background Fits}

We have three types of background in this analysis: cross-feed, $B \bar{B}$, and continuum. The first two give a peaking contribution that is modeled in order to extract the signal yield from 

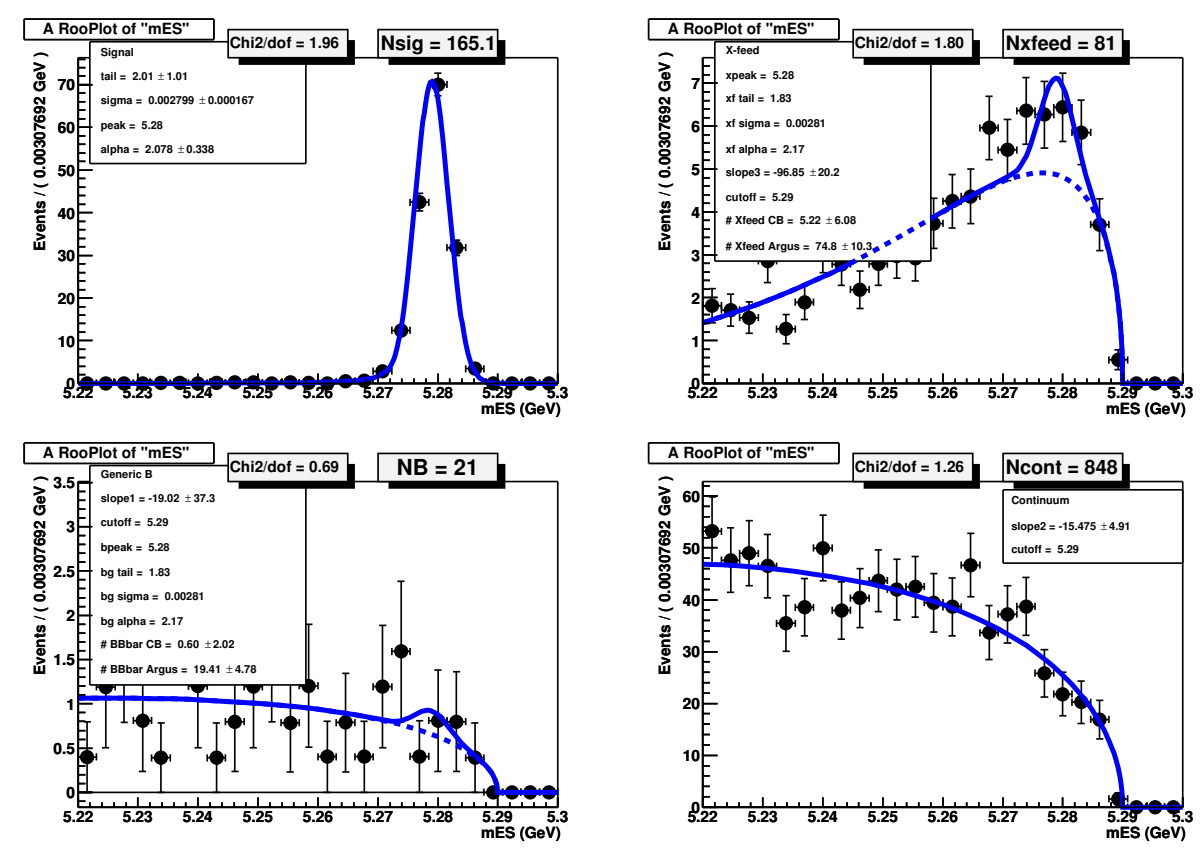

Figure 26: Individual Monte Carlo $m_{\mathrm{ES}}$ plots for 1.1-1.5 GeV mass bin for $\bar{b}$ events: top left signal, top right cross-feed, bottom left $B \bar{B}$, and bottom right continuum.

\begin{tabular}{|c|c|c|c|}
\hline$M_{X s}(\mathrm{GeV})$ & $\alpha$ & $\sigma(\mathrm{MeV})$ & $n$ \\
\hline $0.6-1.1$ & $2.74 \pm 0.12$ & $2.28 \pm 0.49$ & $2.57 \pm 1.60$ \\
$1.1-1.5$ & $2.79 \pm 0.17$ & $2.09 \pm 0.33$ & $1.85 \pm 0.94$ \\
$1.5-1.9$ & $2.86 \pm 0.23$ & $2.32 \pm 0.47$ & $1.03 \pm 0.74$ \\
$1.9-2.3$ & $2.89 \pm 0.35$ & $2.35 \pm 1.38$ & $0.76 \pm 0.89$ \\
\hline Weighted Average & $2.78 \pm 0.09$ & $2.20 \pm 0.23$ & $1.22 \pm 0.47$ \\
\hline
\end{tabular}

Table 16: Values and error weighted average of truth-matched Crystal Ball parameters (both $b$ and $\bar{b}$ events fit together). See Appendix B for the Crystal Ball definition and the description of the free parameters. 

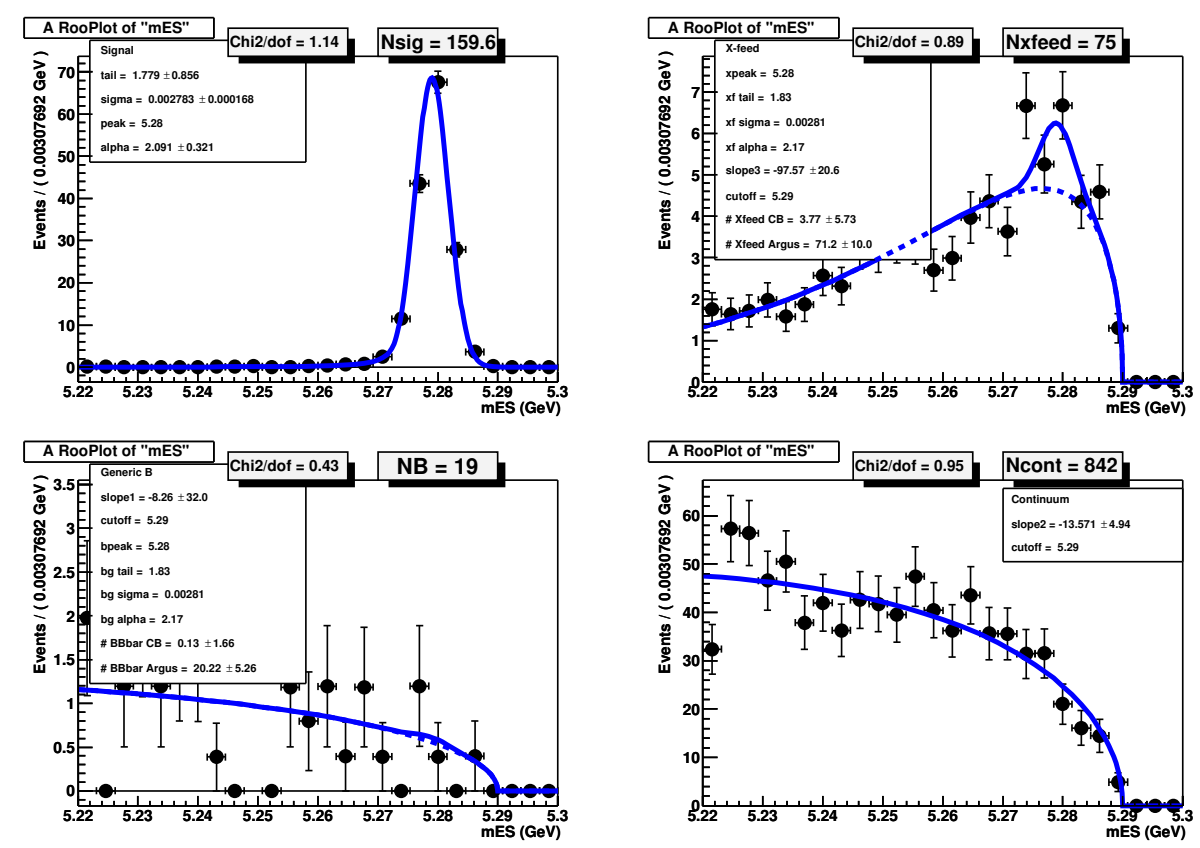

Figure 27: Individual Monte Carlo $m_{\mathrm{ES}}$ plots for $b$ events in the 1.1-1.5 GeV mass bin: top left signal, top right cross-feed, bottom left $B \bar{B}$, and bottom right continuum.

data. In the following, the procedure adopted to fit the backgrounds is described.

\section{Cross-feed and Generic $B$ Fitting (Peaking Background)}

Examples of fits to these two background components can be see in Figure 26 top right (cross-feed) and bottom left $(B \bar{B})$, and Figure 28 for a combined fit of cross-feed and $B \bar{B}$.

Since they have different shapes, the fitting of the combined cross-feed and generic $B \bar{B}$ background with a single Argus and Crystal Ball function must be justified. This is done in Appendix C.

\section{Continuum Fitting}

The continuum background is fit with an Argus function (see Figure 26 bottom right) whose shape is defined from Monte Carlo. We compare equivalent amount of continuum Monte Carlo samples and off-resonance data (see Figure 25). Since they agree, we can use the continuum Monte Carlo to define our continuum Argus functions for the final fits. 


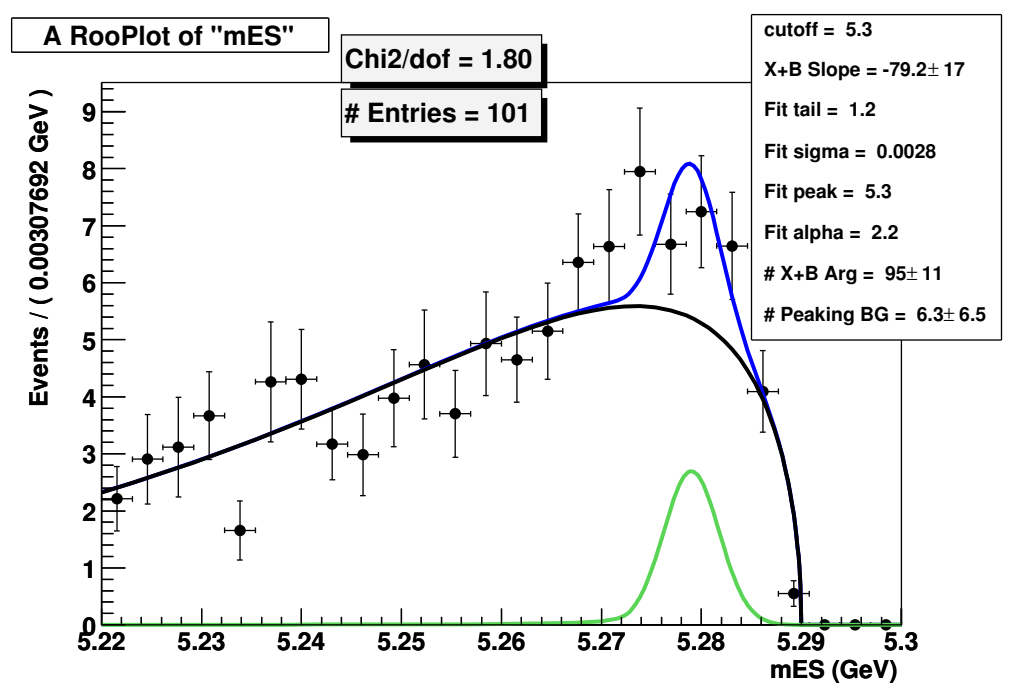

Figure 28: Peaking Monte Carlo (Xfeed $+B \bar{B}$ ) background untagged $m_{\mathrm{ES}}$ plot for 1.1-1.5 $\mathrm{GeV}$ mass for $\bar{b}$ events.
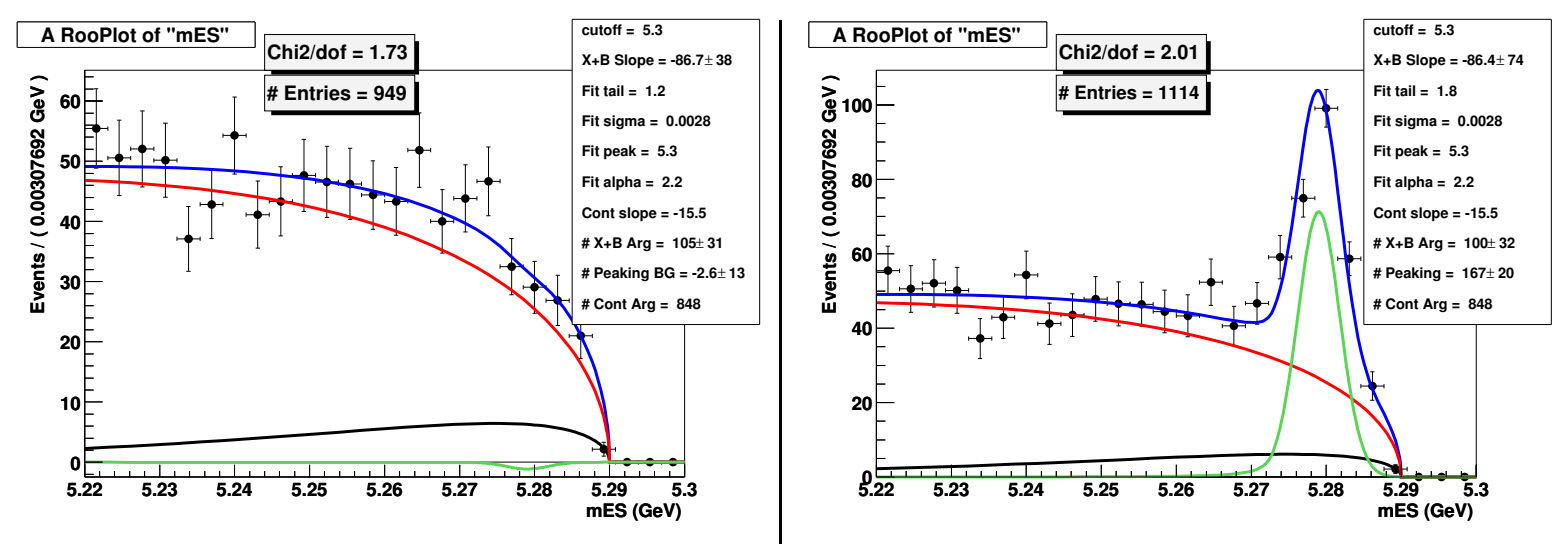

Figure 29: Cross-feed, $B \bar{B}$, and continuum combined fit(left), and full Monte Carlo fit (right), for the 1.1-1.5 GeV mass bin for $\bar{b}$ events.

\subsubsection{Full Fits}

Finally we perform a fit to the full signal plus backgrounds. We simply add the truthmatched signal Monte Carlo to our background samples and fit again (see Figure 29). The difference in the peaking components between the full fit and the peaking background fit 
should be our signal. See Table 17 for the expected yields in our nine categories. This is the same fit we will be used for data.

\begin{tabular}{|l|l|c|c|c|}
\hline Category & Lepton-Tag & $\begin{array}{c}\text { \# Peaking } \\
\text { Background }\end{array}$ & $\begin{array}{c}\text { \# Peaking Signal + } \\
\text { Background }\end{array}$ & \# MC Signal \\
\hline \hline$M_{X s}=0.6-1.1 \mathrm{GeV}$ & Tagged & $3 \pm 3$ & $54 \pm 8$ & 50 \\
& Untagged & $18 \pm 11$ & $435 \pm 30$ & 392 \\
\hline$M_{X s}=1.1-1.5 \mathrm{GeV}$ & Tagged & $0 \pm 2$ & $23 \pm 6$ & 23 \\
& Untagged & $5 \pm 6$ & $159 \pm 20$ & 162 \\
\hline$M_{X s}=1.5-1.9 \mathrm{GeV}$ & Tagged & $2 \pm 3$ & $17 \pm 5$ & 15 \\
& Untagged & $8 \pm 8$ & $91 \pm 20$ & 05 \\
\hline$M_{X s}=1.9-2.3 \mathrm{GeV}$ & Tagged & $1 \pm 3$ & $9 \pm 5$ & 8 \\
& Untagged & $20 \pm 13$ & $67 \pm 28$ & 44 \\
\hline$M_{X s}=0.6-2.3 \mathrm{GeV}$ & Tagged & $5 \pm 6$ & $101 \pm 12$ & 94 \\
& Untagged & $44 \pm 19$ & $734 \pm 49$ & 697 \\
\hline$B^{0}$ & Tagged & $3 \pm 4$ & $55 \pm 8$ & 51 \\
& Untagged & $11 \pm 13$ & $420 \pm 34$ & 393 \\
\hline$B^{+}$decay & Tagged & $-1 \pm 2$ & $16 \pm 5$ & 17 \\
with $K_{S}$ & Untagged & $8 \pm 9$ & $133 \pm 24$ & 116 \\
\hline$B^{+}$decay & Tagged & $3 \pm 4$ & $30 \pm 7$ & 27 \\
with $K^{+}$ & Untagged & $17 \pm 11$ & $134 \pm 22$ & 125 \\
\hline
\end{tabular}

Table 17: Estimated peaking background (cross-feed and $B \bar{B}$ ) from Monte Carlo samples, expected peaking component from the Monte Carlo full fit, and actual number of Monte Carlo truth-matched signal events in the full fit for our nine data categories. The "MC Signal" plus the "Peaking Background" bins equal "Peaking Signal + Background" bins within error. The $b$ and $\bar{b}$ events have been averaged, as this is only to test that the fitting method works.

\subsection{Results from Monte Carlo Events}

Results from the Monte Carlo fits to the peaking background and full data set are reported in Table 18. They are split into the two $C P$ states $(b$ and $\bar{b})$. 


\begin{tabular}{|l||c|c||c|c|}
\hline \multicolumn{5}{|c|}{ Expectations from MC events } \\
\multicolumn{1}{|c|}{$M_{X s}(\mathrm{GeV})$} & $\bar{b}$ & $b$ & $\bar{b}$ & $b$ \\
\hline \hline \multicolumn{5}{|c|}{ Lepton-tagged events } \\
\hline $0.6-1.1$ & $1.3 \pm 2.7$ & $4.4 \pm 2.3$ & $51.1 \pm 8.0$ & $55.9 \pm 8.3$ \\
$1.1-1.5$ & $-0.3 \pm 2.0$ & $0.5 \pm 1.9$ & $22.2 \pm 5.7$ & $21.6 \pm 5$. \\
$1.5-1.9$ & $1.9 \pm 2.8$ & $2.2 \pm 2.7$ & $17.6 \pm 5.0$ & $16.1 \pm 5.0$ \\
$1.9-2.3$ & $-0.9 \pm 3.1$ & $1.5 \pm 3.4$ & $5.3 \pm 4.9$ & $8.0 \pm 4.5$ \\
\hline $0.6-2.3$ & $1.8 \pm 5.9$ & $8.2 \pm 5.7$ & $98 \pm 12$ & $104 \pm 12$ \\
\hline$B^{0}$ & $1.2 \pm 3.9$ & $4.0 \pm 3.8$ & $52.9 \pm 8.6$ & $56.4 \pm 8.4$ \\
$B^{ \pm}\left(K^{ \pm}\right)$ & $3.0 \pm 3.6$ & $3.9 \pm 3.6$ & $29.0 \pm 6.6$ & $31.3 \pm 7.0$ \\
$B^{ \pm}\left(K^{0}\right)$ & $-2.19 \pm 2.3$ & $0.2 \pm 2.0$ & $16.5 \pm 5.5$ & $15.0 \pm 4.9$ \\
\hline \hline \multicolumn{5}{|c|}{ Lepton-untagged events } \\
\hline $0.6-1.1$ & $22 \pm 11$ & $14 \pm 10$ & $434 \pm 31$ & $435 \pm 29$ \\
$1.1-1.5$ & $6.3 \pm 6.5$ & $4.9 \pm 5.9$ & $167 \pm 20$ & $152 \pm 20$ \\
$1.5-1.9$ & $10.9 \pm 9.0$ & $4.9 \pm 7.3$ & $91 \pm 21$ & $90 \pm 19$ \\
$1.9-2.3$ & $18 \pm 13$ & $21 \pm 13$ & $22 \pm 23$ & $69 \pm 25$ \\
\hline $0.6-2.3$ & $40 \pm 19$ & $48 \pm 19$ & $714 \pm 50$ & $754 \pm 48$ \\
\hline$B^{0}$ & $18 \pm 13$ & $21 \pm 13$ & $419 \pm 34$ & $420 \pm 33$ \\
$B \pm\left(K^{ \pm}\right)$ & $14 \pm 11$ & $20 \pm 11$ & $180 \pm 28$ & $180 \pm 26$ \\
$B^{ \pm}\left(K^{0}\right)$ & $9.0 \pm 8.8$ & $6.8 \pm 8.4$ & $113 \pm 22$ & $150 \pm 22$ \\
\hline
\end{tabular}

Table 18: Monte Carlo peaking background and peaking signal + background events as a function of the hadronic mass bins for lepton-tagged and untagged events. The quoted errors are just statistical errors. 


\section{$8 \quad \mathrm{BF}\left(B \rightarrow K^{*} \gamma\right)$ Cross Check}

How are you doing...

Before looking at our expected signal in data, we would like to be able to cross check our selection and fitting technique. Using the off-resonance data we can compare continuum Monte Carlo to data for background shapes. But for signal shapes we must look at a similar decay type to the one in which we are interested. To do this cross-check we measure the branching fraction of the four $B \rightarrow K^{*} \gamma$ modes: $K^{* \pm} \rightarrow K^{0} \pi^{ \pm}, K^{* \pm} \rightarrow K^{ \pm} \pi^{0}$, $K^{* 0} \rightarrow K^{ \pm} \pi^{\mp}$, and $K^{* 0} \rightarrow K^{0} \pi^{0}$. In order to do that, we devise a $B \rightarrow K^{*} \gamma$ dedicated selection, using the same variables used for the $b \rightarrow \mathrm{s} \gamma$ analysis but optimizing the cuts for these signal events. The fitting technique is the same as described in Section 7. In the following, we first describe which are the new optimized cuts for the $B \rightarrow K^{*} \gamma$ final state, then we show the branching fraction results.

\subsection{Selection Differences}

Only four cuts are changed between the $B \rightarrow X s \gamma$, and the $B \rightarrow K^{*} \gamma$ modes. Having a higher signal to background ratio, we are able to open up the $\Delta E^{*}$, neural net, and $\cos \theta_{B^{*}}$ cuts. We also do not require a minimum daughter momentum for the $K^{*}$ region. In addition, we are able to use a stricter truth-matching definition in this region. The difference between the true and reconstructed hadronic mass is required to be within $25 \mathrm{MeV}$ instead of $50 \mathrm{MeV}$ as described in Appendix A.

For the $K^{*}$ region our new cuts are: $-0.15<\Delta E^{*}<-0.1 \mathrm{GeV}$, neural net output $>0.3$, and $\left|\cos \theta_{B^{*}}\right|<.85$ (see Figure 30). Table 19 shows the chosen decay mode versus true decay mode for all $K^{*}$ candidates after the selection and the best candidate selections are applied. 



Figure 30: $\Delta E^{*},\left|\cos \theta_{B^{*}}\right|$, and neural net output distributions for the $K^{*}$ region.

\begin{tabular}{|c|c|c|c|c|}
\hline & $K^{* \pm} \rightarrow K^{0} \pi^{ \pm}$ & $K^{* \pm} \rightarrow K^{ \pm} \pi^{0}$ & $K^{* 0} \rightarrow K^{ \pm} \pi^{\mp}$ & $K^{* 0} \rightarrow K^{0} \pi^{0}$ \\
\hline$K^{* \pm} \rightarrow K^{0} \pi^{ \pm}$ & 99.9 & 0.0789 & 0 & 0 \\
$K^{* \pm} \rightarrow K^{ \pm} \pi^{0}$ & 0.253 & 99.7 & 0 & 0 \\
$K^{* 0} \rightarrow K^{ \pm} \pi^{\mp}$ & 0 & 0 & 99.8 & 0.214 \\
$K^{* 0} \rightarrow K^{0} \pi^{0}$ & 0 & 0 & 3.7 & 96.3 \\
\hline
\end{tabular}

Table 19: Chosen decay mode versus true decay mode for all $K^{*}$ candidates (in percent). 


\subsection{Fitting the $K^{*}$}

The fitting of the $K^{*}$ follows the same procedure as in Section 7 . The average weighted Crystal Ball shape parameters (see Appendix B) among all the $K^{*}$ final states are: $\alpha=$ $2.25 \pm 0.22, \sigma=2.71 \pm 0.07 \mathrm{MeV}, n=2.20 \pm 0.96$.

In Figure 31 we show the individual Monte Carlo $m_{\mathrm{ES}}$ plots for all four $K^{*}$ modes combined, while in Figure 32 we show the full signal and background fits for the combined $K^{*}$ modes for data and Monte Carlo events, so that we might compare data, Monte Carlo differences. We have also compared the $\Delta E^{*}$ distribution for data and Monte Carlo; this can be seen in Figure 33.
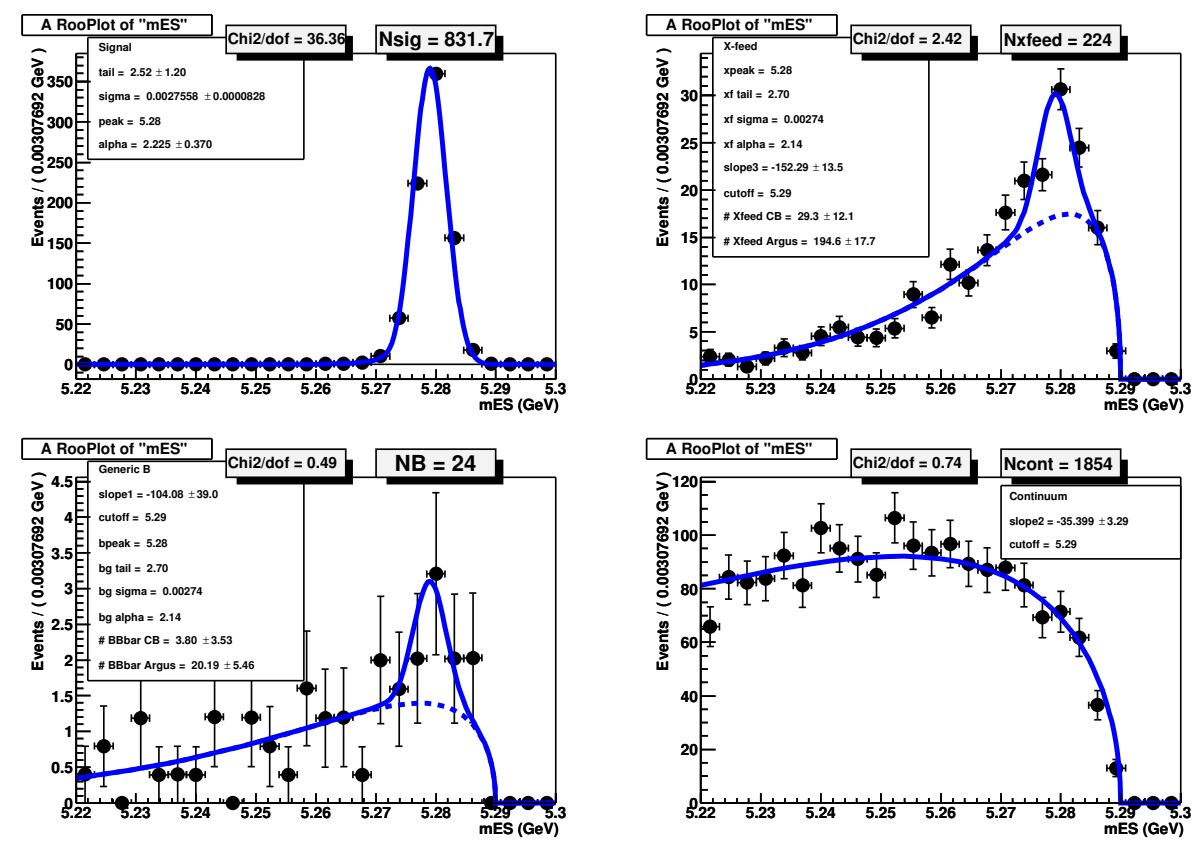

Figure 31: Individual Monte Carlo $m_{\mathrm{ES}}$ plots for all four $K^{*}$ modes combined: top left signal, top right Xfeed, bottom left $B \bar{B}$, and bottom right continuum. 

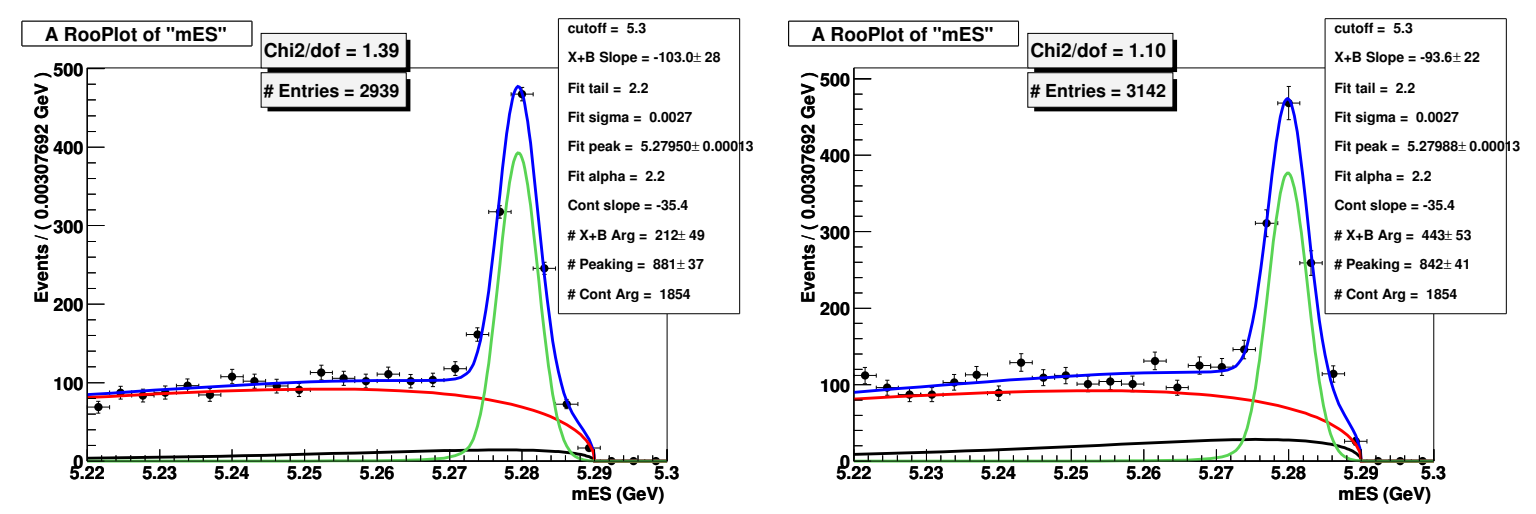

Figure 32: Full signal + background fits for the combined $K^{*}$ modes; left Monte Carlo, right data.

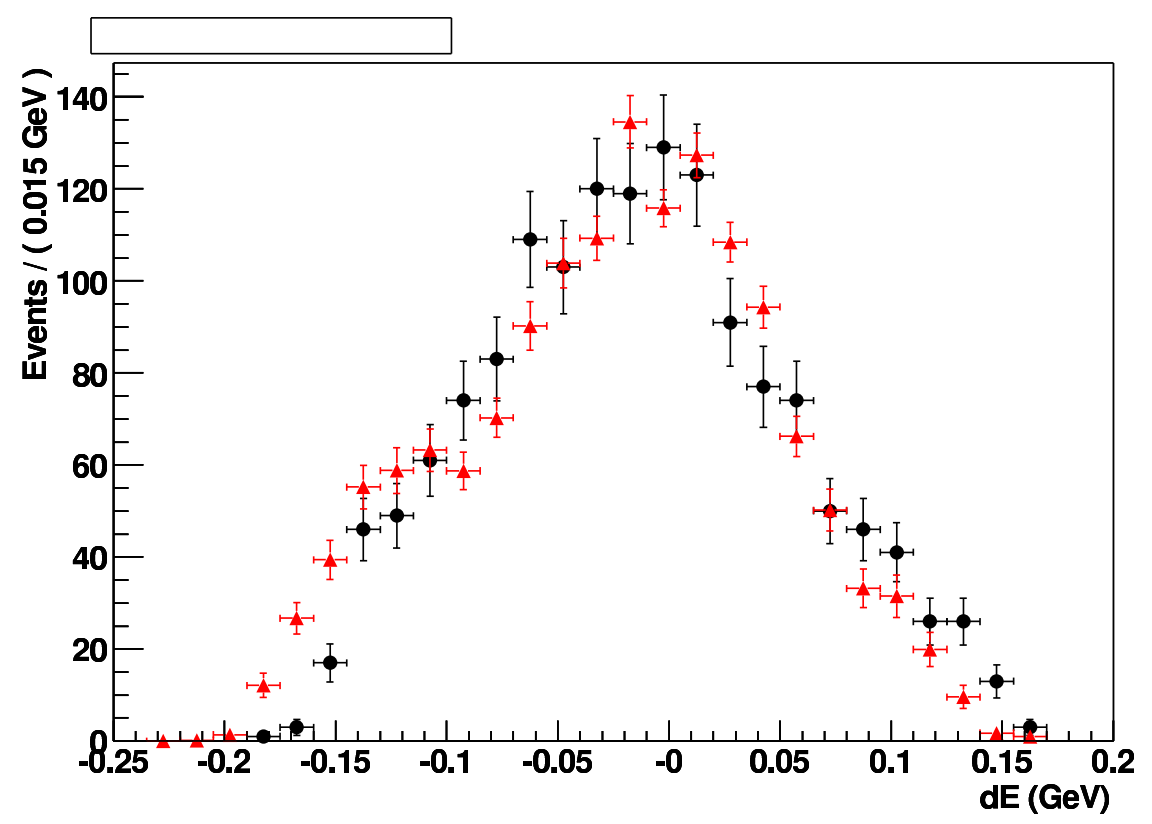

Figure 33: Overlay of Monte Carlo(triangles) and data(circles) for $\Delta E^{*}$. All cuts except our $\Delta E^{*}$ cut have been applied and $m_{\mathrm{ES}}$ asked to be greater than $5.271 \mathrm{GeV}$. 


\section{3 $\quad K^{*}$ Branching Fractions}

$K^{*}$ Branching fractions are determined from the differences in peaking component in the final data fit, and the peaking component of the Monte Carlo background fits. The final branching fraction results can be found in Table 20. They are in good agreement with current 2003 PDG averages of $3.8 \pm 0.5 \times 10^{-5}$ for charged B's and $4.3 \pm 0.4 \times 10^{-5}$ for neutral B's. This cross-checks that our selection and fitting technique work.

\begin{tabular}{|c|c|c|c|c|}
\hline Mode & $\begin{array}{c}\text { \# of BG } \\
\text { Subtracted Signal }\end{array}$ & $\begin{array}{c}\text { Signal Efficiency } \\
(\%)\end{array}$ & $\begin{array}{c}\text { BF } \\
\text { X } 10^{-5}\end{array}$ & $\begin{array}{c}\text { Statistical Error } \\
\text { X } 10^{-5}\end{array}$ \\
\hline$B^{ \pm} \rightarrow K^{* \pm} \gamma, K^{* \pm} \rightarrow K^{0} \pi^{ \pm}$ & 125 & 18.9 & 3.35 & 0.45 \\
$B^{ \pm} \rightarrow K^{* \pm} \gamma, K^{* \pm} \rightarrow K^{ \pm} \pi^{0}$ & 113 & 11.1 & 3.43 & 0.63 \\
$B^{0} \rightarrow K^{* 0} \gamma, K^{* 0} \rightarrow K^{ \pm} \pi^{\mp}$ & 518 & 20.4 & 4.28 & 0.24 \\
$B^{0} \rightarrow K^{* 0} \gamma, K^{* 0} \rightarrow K^{0} \pi^{0}$ & 40 & 10.4 & 3.88 & 1.06 \\
\hline
\end{tabular}

Table 20: Final branching fractions and statistical errors for the four reconstructed $K^{*}$ modes for the current analysis. 


\section{$9 \quad$ Systematic Uncertainties}

...Fine, how are you?

All systematic errors cancel by definition in the measurement of the $C P$ asymmetry, except for systematic effects which are different for $b$ and $\bar{b}$ final states. The effect of these differences is to fake a $C P$ asymmetry.

In the following sections, we will describe the fake $C P$ asymmetry due to detector biases which affects the signal events. All other systematic effects are negligible and can be found in Appendix D. These include: detector biases of the mistagged events and the corresponding asymmetry, $C P$ asymmetry in background events (cross-feed, $B \bar{B}$, and continuum), and possible contributions to the $C P$ asymmetry due to other sources .

\section{1 $C P$ Asymmetry in Signal Events Due to Efficiency Biases}

The detector efficiency is not symmetric for positive and negative charged tracks. Tracking efficiency differences can arise from the opposite curvature of the tracks in the solenoid field. In the Drift Chamber this couples to the drift electron direction, which is distorted by the Lorentz angle effect. This can affect both the track reconstruction and the $\mathrm{dE} / \mathrm{dx}$ particle identification information. The other major source of asymmetries is the different strong interaction rates of positive and negative particles, particularly charged kaons. This leads to tracking efficiency asymmetries and particle identification asymmetries.

To estimate the contribution to the $C P$ asymmetry due to the particle efficiency asymmetry for charged pions and kaons we study the on-resonance and off-resonance data. The on-resonance data are divided in the following categories:

- On-resonance sideband sample: The on-resonance sideband sample defined by $\left|\Delta E^{*}\right|<0.3 \mathrm{GeV}$ and $m_{\mathrm{ES}}<5.27 \mathrm{GeV}$ or $0.11<\left|\Delta E^{*}\right|<0.3 \mathrm{GeV}$ and $m_{\mathrm{ES}}>5.27 \mathrm{GeV}$, 
and releasing completely the Neural Network cut to gain in statistics.

- On-resonance continuum enriched sample: The on-resonance continuum enriched sample is defined by $\left|\Delta E^{*}\right|<0.3 \mathrm{GeV}$ and $m_{\mathrm{ES}}<5.27 \mathrm{GeV}$ or $0.11<\left|\Delta E^{*}\right|<$ $0.3 \mathrm{GeV}$ and $m_{\mathrm{ES}}>5.27 \mathrm{GeV}$, and $\mathrm{NN}<0.4$.

- On-resonance continuum suppressed sample: The on-resonance continuum suppressed sample is defined by $\left|\Delta E^{*}\right|<0.3 \mathrm{GeV}$ and $m_{\mathrm{ES}}<5.27 \mathrm{GeV}$ or $0.11<\left|\Delta E^{*}\right|<$ $0.3 \mathrm{GeV}$ and $m_{\mathrm{ES}}>5.27 \mathrm{GeV}$, and $\mathrm{NN}>0.4$.

- On-resonance antiveto sample: the on-resonance antiveto sample is defined by on-resonance data cut out by the $\pi^{0}$ and $\eta$ vetoes (see section 6.4).

All samples are lepton-untagged. The measured flavor asymmetry, defined previously as Equation 55:

$$
A_{C P}^{\text {measured }}=\frac{n(b \rightarrow s \gamma)-n(\bar{b} \rightarrow \bar{s} \gamma)}{n(b \rightarrow s \gamma)+n(\bar{b} \rightarrow \bar{s} \gamma)}
$$

Results for the four on-resonance samples and the off-resonance data are shown in Table 21 as a function of the hadronic mass.

From Table 21, we learn that there is a non-null asymmetry in all the data samples. However, the on-resonance antiveto sample and the off-resonance data are not statistically significant. All of the on-resonance sideband samples show an asymmetry. The fact that the asymmetry is present both in the continuum enriched and suppressed samples means that it is not due to the asymmetry in the $B \bar{B}$ sample.

The asymmetry in the data is tracked down to have a dependence on the charged kaon momentum. Results are reported in Table 22. The fact that there is a strong asymmetry for low energy kaons is mainly due to the different nuclear cross-section for positive and negative kaons, especially at low momenta. The nuclear cross-sections, are shown in Appendix E. 
As lower momentum kaons are mainly present at higher hadronic masses, there is a higher asymmetry in the on-resonance and off-resonance data at higher hadronic masses.

Finally, we have studied the events with just $K_{S}$ as a function of the $K_{S}$ momentum. Results are reported in Table 23, and show an asymmetry. We assume that it is due to the fact that the $K^{0}$ and $\overline{K^{0}}$ have a different nuclear cross-section.

Before defining the strategy to adopt regarding Monte Carlo events, we need to look at the Monte Carlo events and study any $C P$ asymmetry bias they have. We have used the official recipes from the tracking and PID groups to correct Monte Carlo events. In both cases, look-up tables for positive and negative charged tracks and kaons are provided. These contain the ratio of the tracking efficiency in data and Monte Carlo and the absolute efficiency of our kaon selector. We have tabulated the PID corrections in Appendix D Table 40.

The apparent $C P$ asymmetry in signal Monte Carlo events, corrected by the tracking and PID, is analyzed. Results are shown in Appendix D Table 41 as a function of the hadronic mass bin for lepton-tagged and untagged events, and there is no evidence of bias within the statistical errors.

We have formulated some hypotheses to try to understand the reasons why the Monte Carlo events do not simulate the asymmetry found in data properly. We know that there is not a good hadronic model for kaons in the low momentum region, and $K_{S}$ regeneration is not simulated [37]. We have tried to quantify the asymmetry caused by the different nuclear interaction of charged kaons in the beam-pipe and SVT which is not simulated or taken into account by the tracking and the PID. The study can be found in Appendix E. We show that we manage to explain a negative asymmetry around $0.005-0.01$ with our simple model.

Since Monte Carlo events do not show the same asymmetry as seen in data, we re-weight the Monte Carlo events according to the asymmetry found in data as a function of the kaon momentum. After reweighing signal Monte Carlo as a function of kaon momentum (see 


\begin{tabular}{|c|c|c|c|c|c|c|}
\hline \multirow[b]{2}{*}{$M_{X s}(\mathrm{GeV})$} & \multicolumn{3}{|c|}{ Argus } & \multicolumn{3}{|c|}{ Crystal Ball } \\
\hline & $\bar{b}$ & $b$ & $A_{C P}^{\text {measured }}$ & $\bar{b}$ & $b$ & $A_{C P}^{\text {measured }}$ \\
\hline & \multicolumn{6}{|c|}{ On-resonance sideband data } \\
\hline $0.6-1.1$ & 2376 & 2367 & $0.00 \pm 0.01$ & & & \\
\hline $1.1-1.5$ & 3222 & 3263 & $0.01 \pm 0.01$ & & & \\
\hline $1.5-1.9$ & 7429 & 7208 & $-0.02 \pm 0.01$ & & & \\
\hline $1.9-2.3$ & 15319 & 14669 & $-0.02 \pm 0.01$ & & & \\
\hline $0.6-2.3$ & 28346 & 27447 & $-0.016 \pm 0.004$ & & & \\
\hline$B^{0}$ & 8520 & 8319 & $-0.01 \pm 0.01$ & & & \\
\hline$B \pm\left(K^{ \pm}\right)$ & 6581 & 6417 & $-0.01 \pm 0.01$ & & & \\
\hline$B^{ \pm}\left(K^{0}\right)$ & 4716 & 4620 & $-0.01 \pm 0.01$ & & & \\
\hline & \multicolumn{6}{|c|}{ "On-resonance continuum enriched sample } \\
\hline $0.6-1.1$ & 1140 & 1135 & $0.00 \pm 0.02$ & & & \\
\hline $1.1-1.5$ & 1583 & 1640 & $0.01 \pm 0.02$ & & & \\
\hline $1.5-1.9$ & 3483 & 3405 & $-0.01 \pm 0.01$ & & & \\
\hline $1.9-2.3$ & 6953 & 6593 & $-0.02 \pm 0.01$ & & & \\
\hline $0.6-2.3$ & 13159 & 12773 & $-0.01 \pm 0.01$ & & & \\
\hline & \multicolumn{6}{|c|}{ "On-resonance continuum suppressed sample } \\
\hline $0.6-1.1$ & 1236 & 1172 & $-0.03 \pm 0.02$ & & & \\
\hline $1.1-1.5$ & 1639 & 1623 & $0.00 \pm 0.02$ & & & \\
\hline $1.5-1.9$ & 3946 & 3803 & $-0.02 \pm 0.01$ & & & \\
\hline $1.9-2.3$ & 8366 & 8076 & $-0.02 \pm 0.01$ & & & \\
\hline $0.6-2.3$ & 15187 & 14674 & $-0.02 \pm 0.01$ & & & \\
\hline & \multicolumn{6}{|c|}{ On-resonance antiveto Sample } \\
\hline $0.6-1.1$ & $100 \pm 11$ & $87 \pm 10$ & $-0.07 \pm 0.08$ & $-8.6 \pm 3.6$ & $8.5 \pm 5.1$ & $0.33 \pm 0.28$ \\
\hline $1.1-1.5$ & $355 \pm 21$ & $319 \pm 20$ & $-0.05 \pm 0.04$ & $14.6 \pm 9.2$ & $21.6 \pm 9.0$ & $0.19 \pm 0.36$ \\
\hline $1.5-1.9$ & $664 \pm 28$ & $705 \pm 28$ & $0.03 \pm 0.03$ & $21.6 \pm 9.0$ & $32 \pm 11$ & $-0.12 \pm 0.27$ \\
\hline $1.9-2.3$ & $1545 \pm 28$ & $1524 \pm 28$ & $-0.01 \pm 0.01$ & $23 \pm 14$ & $22 \pm 16$ & $-0.02 \pm 0.47$ \\
\hline $0.6-2.3$ & $2664 \pm 55$ & $2633 \pm 55$ & $-0.01 \pm 0.01$ & $62 \pm 21$ & $79 \pm 22$ & $0.12 \pm 0.22$ \\
\hline & \multicolumn{6}{|c|}{ Off-resonance Data } \\
\hline $0.6-1.1$ & 1282 & 1300 & $0.01 \pm 0.02$ & & & \\
\hline $1.1-1.5$ & 940 & 1111 & $-0.07 \pm 0.02$ & & & \\
\hline $1.5-1.9$ & 2403 & 2291 & $0.03 \pm 0.02$ & & & \\
\hline $1.9-2.3$ & 3959 & 4455 & $-0.07 \pm 0.02$ & & & \\
\hline $0.6-2.3$ & 8576 & 9157 & $-0.03 \pm 0.01$ & & & \\
\hline
\end{tabular}

Table 21: Fitted number of events in Argus and peaking the Crystal Ball fitted distributions in on-resonance sideband data, on-resonance continuum enriched and suppressed data, antiveto sample and off-resonance data for $b$ and $\bar{b}$ flavors. The corresponding $C P$ asymmetry is computed. 


\begin{tabular}{|c||c|c|c|}
\hline \multicolumn{1}{|c||}{} & \multicolumn{3}{c|}{ Argus } \\
$P_{K}(\mathrm{GeV})$ & $\bar{b}$ & $b$ & $A_{C P}$ \\
\hline \hline \multicolumn{1}{|c||}{} & \multicolumn{3}{c|}{ K On-resonance sideband data } \\
\hline $0.2-0.4$ & $1092 \pm 33$ & $1061 \pm 33$ & $-0.014 \pm 0.022$ \\
$0.4-0.6$ & $3633 \pm 60$ & $3464 \pm 59$ & $-0.024 \pm 0.012$ \\
$0.6-0.8$ & $3283 \pm 57$ & $3061 \pm 55$ & $-0.035 \pm 0.013$ \\
$0.8-1.0$ & $2346 \pm 48$ & $2138 \pm 46$ & $-0.046 \pm 0.015$ \\
$1.0-1.2$ & $2280 \pm 48$ & $2285 \pm 48$ & $0.001 \pm 0.015$ \\
$1.2-2.0$ & $8526 \pm 92$ & $8523 \pm 92$ & $0.000 \pm 0.007$ \\
$2.0-3.0$ & $2636 \pm 51$ & $2601 \pm 51$ & $-0.007 \pm 0.013$ \\
$3.0-4.0$ & $542 \pm 23$ & $521 \pm 23$ & $-0.020 \pm 0.030$ \\
\hline
\end{tabular}

Table 22: $C P$ asymmetry dependence on the charged kaon momentum in on-resonance sideband data.

\begin{tabular}{|c||c|c|c|}
\hline \multicolumn{1}{|c||}{} & \multicolumn{3}{c|}{ Argus } \\
$P_{K_{S}}(\mathrm{GeV})$ & $\bar{b}$ & $b$ & $A_{C P}$ \\
\hline \hline \multicolumn{1}{|c||}{} & \multicolumn{3}{c|}{$K_{S}$ On-resonance sideband data } \\
\hline $0.2-0.4$ & $311 \pm 18$ & $300 \pm 17$ & $-0.018 \pm 0.041$ \\
$0.4-0.6$ & $836 \pm 29$ & $780 \pm 28$ & $-0.035 \pm 0.025$ \\
$0.6-0.8$ & $823 \pm 29$ & $752 \pm 27$ & $-0.045 \pm 0.025$ \\
$0.8-1.0$ & $743 \pm 27$ & $753 \pm 27$ & $0.007 \pm 0.026$ \\
$1.0-1.2$ & $681 \pm 26$ & $663 \pm 26$ & $-0.013 \pm 0.027$ \\
$1.2-2.0$ & $2501 \pm 50$ & $2462 \pm 50$ & $-0.008 \pm 0.014$ \\
$2.0-3.0$ & $835 \pm 29$ & $812 \pm 28$ & $-0.014 \pm 0.024$ \\
$3.0-4.0$ & $218 \pm 15$ & $196 \pm 14$ & $-0.053 \pm 0.054$ \\
\hline
\end{tabular}

Table 23: $C P$ asymmetry dependence on the $K_{S}$ momentum in on-resonance sideband data. 
Tables 22 and 23), we find a small $A_{C P}$ in our expected result (see Tables 41 and 42 in Appendix D).

The observed differences of the signal Monte Carlo are the shifts $\left(A_{C P}{ }^{\text {Shift }}\right)$ we need to apply on our final measurement. We take the difference assuming that the original fake asymmetry in Monte Carlo is actually zero, and difference from zero is due to the statistical fluctuations. The shifts we need to apply to data are shown in Table 24. The error of the shifts is the sum in quadrature of the statistical error and the error from the variation of of the weights $\pm 1 \sigma$ from Tables 22 and 23 .

Note that for the lepton-tagged sample we need also to attribute an error due to the lepton-tag asymmetry [31]. We attribute a total error of 0.01 for the tagging categories used in our analysis.

\begin{tabular}{|l||c|c|}
\hline$M_{X s}(\mathrm{GeV})$ & $A_{C P \text { tag. }}^{\text {Shift }}$ & $A_{C P \text { untag. }}^{\text {Shift }}$ \\
\hline $0.6-1.1$ & $-0.014 \pm 0.025$ & $-0.014 \pm 0.015$ \\
$1.1-1.5$ & $-0.016 \pm 0.068$ & $-0.016 \pm 0.028$ \\
$1.5-1.9$ & $-0.017 \pm 0.079$ & $-0.016 \pm 0.033$ \\
$1.9-2.3$ & $-0.017 \pm 0.091$ & $-0.017 \pm 0.038$ \\
\hline $0.6-2.3$ & $-0.014 \pm 0.023$ & $-0.014 \pm 0.015$ \\
\hline$B^{0}$ & $-0.006 \pm 0.028$ & $-0.006 \pm 0.014$ \\
$B^{ \pm}\left(K^{ \pm}\right)$ & $0.0041 \pm 0.045$ & $-0.007 \pm 0.018$ \\
$B^{ \pm}\left(K^{0}\right)$ & $-0.026 \pm 0.060$ & $-0.048 \pm 0.026$ \\
\hline
\end{tabular}

Table 24: Shifts due to the fake detector $C P$ asymmetry in reconstructed data signal events as a function of the hadronic mass bins for lepton-tagged $\left(A_{C P} \begin{array}{c}\text { Shift } \\ \text { tag. }\end{array}\right)$ and untagged events $\left(A_{C P \text { untag. }}^{\text {Shift }}\right)$. This is our only systematic error. 


\section{Results}

You need to be really concerned when statements like that come from me.

In this section the determination of the $A_{C P}$ as a function of the hadronic mass is performed. In the following, we describe the formula used for the measurement of $A_{C P}$, and a summary of the inputs for calculating $A_{C P}$. We then present the results from our measurement. We remind ourselves that the formula for calculating $A_{C P}$ from experimental values was defined before as Equation 48:

$$
A_{C P}=\left(A_{C P}^{\text {measured }}-\frac{\Delta D}{2}\right) \frac{1}{<D>}-A_{C P}^{S h i f t} .
$$

\section{1 $A_{C P}(b \rightarrow s \gamma)$ Numerical Inputs from Monte Carlo}

Table 25 shows a summary of the reconstructed number of Monte Carlo $b$ and $\bar{b}$ events events for the categories we study, computed as the difference between the background subtracted Monte Carlo events and the expected peaking contribution from cross-feed and $B \bar{B}$ Monte Carlo events. The relation between the reconstructed number of events, the background subtracted Monte Carlo events and the expected peaking contribution was shown in Section 7. The values for the Monte Carlo samples are already reported in Section 7 and copied here again for clarity.

Table 26 shows a summary of the values of $\Delta D$ and $\langle D\rangle$ obtained from the mistag rates for $b$ and $\bar{b}$ events. Using Equations (49), (50), (51), (52) we derive the relation between $\Delta D$ and $\langle D\rangle$ and the mistag rates, Equations 51 and 52 .

The mistag rates were already derived in Appendix D.1 and reported now again for clarity.

Finally, the detector $A_{C P}$, as derived in the systematic Section 9.1, is reported in Table 27. All the ingredients necessary to compute the $C P$ asymmetry in Monte Carlo are now in place 
and it will be described in the next section.

\begin{tabular}{|c|c|c|c|c|c|c|}
\hline \multicolumn{7}{|c|}{ Expectations from MC events } \\
\hline & \multicolumn{2}{|c|}{ Peaking background events } & \multicolumn{2}{|c|}{ Full MC peaking events } & \multicolumn{2}{|c|}{$n_{\text {recoed }}$} \\
\hline$M_{X s}(\mathrm{GeV})$ & $\bar{b}$ & $b$ & $\bar{b}$ & $b$ & $\bar{b}$ & $b$ \\
\hline \multicolumn{7}{|c|}{$\begin{array}{l}\text { Lepton-tagged events } \\
\text { sed }\end{array}$} \\
\hline $0.6-1.1$ & $1.3 \pm 2.7$ & $4.4 \pm 2.3$ & $51.1 \pm 8.0$ & $55.9 \pm 8.3$ & $49.8 \pm 8.4$ & $51.5 \pm 8.6$ \\
\hline $1.1-1.5$ & $-0.3 \pm 2.0$ & $0.5 \pm 1.9$ & $22.2 \pm 5.7$ & $21.6 \pm 5.5$ & $22.5 \pm 6.0$ & $21.1 \pm 5.8$ \\
\hline $1.5-1.9$ & $1.9 \pm 2.8$ & $2.2 \pm 2.7$ & $17.6 \pm 5.0$ & $16.1 \pm 5.0$ & $15.7 \pm 5.7$ & $13.9 \pm 5.7$ \\
\hline $1.9-2.3$ & $-0.9 \pm 3.1$ & $1.5 \pm 3.4$ & $5.3 \pm 4.9$ & $8.0 \pm 4.5$ & $6.2 \pm 5.8$ & $6.5 \pm 5.6$ \\
\hline $0.6-2.3$ & $1.8 \pm 5.9$ & $8.2 \pm 5.7$ & $98 \pm 12$ & $104 \pm 12$ & $96.2 \pm 13.37$ & $95.8 \pm 13.3$ \\
\hline$B^{0}$ & $1.2 \pm 3.9$ & $4.0 \pm 3.8$ & $52.9 \pm 8.6$ & $56.4 \pm 8.4$ & $51.7 \pm 9.4$ & $52.4 \pm 9.2$ \\
\hline$B^{ \pm}\left(K^{ \pm}\right)$ & $3.0 \pm 3.6$ & $3.9 \pm 3.6$ & $29.0 \pm 6.6$ & $31.3 \pm 7.0$ & $26.0 \pm 7.5$ & $27.4 \pm 7.9$ \\
\hline$B^{ \pm}\left(K^{0}\right)$ & $-2.19 \pm 2.3$ & $0.2 \pm 2.0$ & $16.5 \pm 5.5$ & $15.0 \pm 4.9$ & $18.7 \pm 6.0$ & $14.8 \pm 5.3$ \\
\hline \multicolumn{7}{|c|}{ Lepton-untagged events } \\
\hline $0.6-1.1$ & $22 \pm 11$ & $14 \pm 10$ & $434 \pm 31$ & $435 \pm 29$ & $412 \pm 33$ & $421 \pm 31$ \\
\hline $1.1-1.5$ & $6.3 \pm 6.5$ & $4.9 \pm 5.9$ & $167 \pm 20$ & $152 \pm 20$ & $161 \pm 21$ & $147 \pm 21$ \\
\hline $1.5-1.9$ & $10.9 \pm 9.0$ & $4.9 \pm 7.3$ & $91 \pm 21$ & $90 \pm 19$ & $80 \pm 23$ & $85 \pm 20$ \\
\hline $1.9-2.3$ & $18 \pm 13$ & $21 \pm 13$ & $22 \pm 23$ & $69 \pm 25$ & $4 \pm 26$ & $48 \pm 28$ \\
\hline $0.6-2.3$ & $40 \pm 19$ & $48 \pm 19$ & $714 \pm 50$ & $754 \pm 48$ & $674 \pm 53$ & $706 \pm 52$ \\
\hline$B^{0}$ & $18 \pm 13$ & $21 \pm 13$ & $419 \pm 34$ & $420 \pm 33$ & $401 \pm 36$ & $399 \pm 35$ \\
\hline$B^{ \pm}\left(K^{ \pm}\right)$ & $14 \pm 11$ & $20 \pm 11$ & $180 \pm 28$ & $180 \pm 26$ & $166 \pm 30$ & $160 \pm 28$ \\
\hline$B^{ \pm}\left(K^{0}\right)$ & $9.0 \pm 8.8$ & $6.8 \pm 8.4$ & $113 \pm 22$ & $150 \pm 22$ & $104 \pm 24$ & $143 \pm 24$ \\
\hline
\end{tabular}

Table 25: Monte Carlo peaking background, full Monte Carlo peaking events and reconstructed number of events from the difference of the full Monte Carlo peaking events and Monte Carlo peaking background as a function of the hadronic mass bins for lepton-tagged and untagged events. The quoted errors are just statistical errors.

\subsection{Expected $A_{C P}(b \rightarrow s \gamma)$ in Monte Carlo Events}

The $A_{C P}$ in Monte Carlo events, using Equation (48), and using the input numbers from Tables 25, 26 and 27, are shown in Table 28. As can be observed in Table 28, we do not expect an asymmetry in Monte Carlo events.

\subsection{Data Fits}

Figure 34 shows the background subtracted fits to the data for $M_{X s}=0.6-2.3 \mathrm{GeV}$. The results for all the categories we study are shown in Table 29. The expected peaking 


\begin{tabular}{|l|c|c|c|c|}
\hline \multicolumn{5}{|c|}{ Lepton-tagged events } \\
\hline$M_{X s}(\mathrm{GeV})$ & $\omega$ & $\bar{\omega}$ & $\Delta D$ & $<D>$ \\
\hline $0.6-1.1$ & $0.0017 \pm 0.0009$ & $0.006 \pm 0.002$ & $0.004 \pm 0.002$ & $0.996 \pm 0.001$ \\
$1.1-1.5$ & $0.013 \pm 0.007$ & $0.014 \pm 0.008$ & $0.001 \pm 0.011$ & $0.987 \pm 0.005$ \\
$1.5-1.9$ & - & $0.012 \pm 0.0009$ & $0.0120 \pm 0.0009$ & $0.9940 \pm 0.0005$ \\
$1.9-2.3$ & $0.015 \pm 0.011$ & - & $-0.015 \pm 0.011$ & $0.993 \pm 0.006$ \\
\hline $0.6-2.3$ & $0.0021 \pm 0.0009$ & $0.007 \pm 0.002$ & $0.005 \pm 0.002$ & $0.995 \pm 0.001$ \\
\hline$B^{0}$ & $0.004 \pm 0.002$ & $0.012 \pm 0.003$ & $0.008 \pm 0.004$ & $0.992 \pm 0.002$ \\
$B^{ \pm}\left(K^{ \pm}\right)$ & - & - & - & - \\
$B^{ \pm}\left(K^{0}\right)$ & $0.006 \pm 0.003$ & $0.002 \pm 0.002$ & $-0.004 \pm 0.004$ & $0.996 \pm 0.002$ \\
\hline \hline \multicolumn{5}{|c|}{ Lepton-untagged events } \\
\hline$M_{X s}(\mathrm{GeV})$ & $\omega$ & $\bar{\omega}$ & $\Delta D$ & $<D>$ \\
\hline $0.6-1.1$ & $0.0049 \pm 0.0005$ & $0.0052 \pm 0.0008$ & $0.0003 \pm 0.0009$ & $0.9950 \pm 0.0005$ \\
$1.1-1.5$ & $0.012 \pm 0.003$ & $0.008 \pm 0.003$ & $-0.004 \pm 0.004$ & $0.990 \pm 0.002$ \\
$1.5-1.9$ & $0.004 \pm 0.002$ & $0.007 \pm 0.004$ & $0.003 \pm 0.004$ & $0.995 \pm 0.002$ \\
$1.9-2.3$ & $0.014 \pm 0.004$ & $0.010 \pm 0.005$ & $-0.004 \pm 0.006$ & $0.988 \pm 0.003$ \\
\hline $0.6-2.3$ & $0.0052 \pm 0.0005$ & $0.0056 \pm 0.0007$ & $-0.004 \pm 0.006$ & $0.988 \pm 0.003$ \\
\hline$B^{0}$ & $0.0073 \pm 0.0008$ & $0.0072 \pm 0.0011$ & $0.000 \pm 0.001$ & $0.9928 \pm 0.0007$ \\
$B \pm\left(K^{ \pm}\right)$ & $0.0014 \pm 0.0005$ & $0.0009 \pm 0.0006$ & $0.000 \pm 0.001$ & $0.9989 \pm 0.0004$ \\
$B^{ \pm}\left(K^{0}\right)$ & $0.006 \pm 0.001$ & $0.007 \pm 0.002$ & $0.001 \pm 0.002$ & $0.994 \pm 0.001$ \\
\hline
\end{tabular}

Table 26: The dilution difference, $\Delta D$, and average, $\langle D\rangle$, and mis-tag rates $(\omega$ and $\bar{\omega}$ for $b$ and $\bar{b}$ events, respectively) as derived from signal Monte Carlo events in Section D.1 for lepton-tagged and untagged events. Where there is - means that there were not enough statistics.

\begin{tabular}{|l||c|c|}
\hline$M_{X s}(\mathrm{GeV})$ & $A_{C P \text { tag. }}^{\text {Shift }}$ & $A_{C P \text { Shift }}^{\text {Shig. }}$ \\
\hline $0.6-1.1$ & $-0.014 \pm 0.025$ & $-0.014 \pm 0.015$ \\
$1.1-1.5$ & $-0.016 \pm 0.068$ & $-0.016 \pm 0.028$ \\
$1.5-1.9$ & $-0.017 \pm 0.079$ & $-0.016 \pm 0.033$ \\
$1.9-2.3$ & $-0.017 \pm 0.091$ & $-0.017 \pm 0.038$ \\
\hline $0.6-2.3$ & $-0.014 \pm 0.023$ & $-0.014 \pm 0.015$ \\
\hline$B^{0}$ & $-0.006 \pm 0.028$ & $-0.006 \pm 0.014$ \\
$B^{ \pm}\left(K^{ \pm}\right)$ & $0.0041 \pm 0.045$ & $-0.007 \pm 0.018$ \\
$B^{ \pm}\left(K^{0}\right)$ & $-0.026 \pm 0.060$ & $-0.048 \pm 0.026$ \\
\hline \hline
\end{tabular}

Table 27: Shifts due to the fake detector $C P$ asymmetry in reconstructed data signal events as a function of the hadronic mass bins for lepton-tagged $\left(A_{C P}{ }^{\text {Shift }}{ }_{\text {tag. }}\right)$ and untagged events $\left(A_{C P}{ }_{\text {Shift }}\right.$ untag. $)$. 


\begin{tabular}{|l||c|c|}
\hline$M_{X s}(\mathrm{GeV})$ & $A_{C P_{\text {tag. }}}^{M C}$ & $A_{C P_{\text {untag. }}^{M C}}^{M C}$ \\
\hline $0.6-1.1$ & $0.02 \pm 0.12$ & $0.01 \pm 0.05$ \\
$1.1-1.5$ & $-0.03 \pm 0.19$ & $-0.04 \pm 0.10$ \\
$1.5-1.9$ & $-0.06 \pm 0.27$ & $0.03 \pm 0.18$ \\
$1.9-2.3$ & $0.02 \pm 0.64$ & $0.85 \pm 1.00$ \\
\hline $0.6-2.3$ & $0.00 \pm 0.10$ & $0.02 \pm 0.05$ \\
\hline$B^{0}$ & $0.00 \pm 0.13$ & $0.00 \pm 0.06$ \\
$B^{ \pm}\left(K^{ \pm}\right)$ & $0.03 \pm 0.20$ & $-0.02 \pm 0.13$ \\
$B^{ \pm}\left(K^{0}\right)$ & $-0.12 \pm 0.24$ & $0.16 \pm 0.14$ \\
\hline
\end{tabular}

Table 28: Expected $A_{C P}$ (in Monte Carlo) as a function of the hadronic mass bins. The $A_{C P}$ is not corrected by the mistag rate and the detector shift. The quoted errors are just statistical errors.

contribution from cross-feed and $B \bar{B}$ Monte Carlo are used for background subtraction. We have now all the information needed in order to measure the direct $C P$ violation in data.

\subsection{Measured $A_{C P}(b \rightarrow s \gamma)$ in Data Events}

The measured $A_{C P}$ in data events, using Equation (48), and using the input numbers from Tables 26, 27 and 29 is shown in Table 30 for all of our categories. The same results described in Table 30 are also presented in Figure 35 for clarity.

The measured value of the $A_{C P}$ for $M_{X s}=0.6-2.3 \mathrm{GeV}$ is our most significant result, and is consistent with zero within $5.0 \%$ statistical error and $1.5 \%$ systematical error. The breakdown in categories show that the results are all consistent with zero within 1-2 $\sigma$. We note the opposite behavior of the last two categories $\left(B^{ \pm}\left(K^{ \pm}\right)\right.$and $\left.B^{ \pm}\left(K^{0}\right)\right)$. These two categories are $2.3 \sigma$ apart and represent the values of the direct $C P$ asymmetry for the charged $B$ categories split into charged and neutral kaons. When we further investigate this deviation and find that:

- The asymmetry is only seen in hadronic masses greater than $1.1 \mathrm{GeV}$. There is no asymmetry in the $\mathrm{K}^{*}$ region. 


\begin{tabular}{|c|c|c|c|c|}
\hline \multicolumn{5}{|c|}{ Data events } \\
\hline & \multicolumn{2}{|c|}{ Full MC peaking events } & \multicolumn{2}{|c|}{$n_{\text {recoed }}$} \\
\hline$M_{X s}(\mathrm{GeV})$ & $b$ & $\bar{b}$ & $b$ & $\bar{b}$ \\
\hline \multicolumn{5}{|c|}{ Lepton-tagged events } \\
\hline $0.6-1.1$ & $45.2 \pm 8.3$ & $44.9 \pm 8.8$ & $40.8 \pm 8.6$ & $43.6 \pm 9.2$ \\
\hline $1.1-1.5$ & $21.3 \pm 5.2$ & $19.7 \pm 5.9$ & $20.8 \pm 5.5$ & $20 \pm 6.2$ \\
\hline $1.5-1.9$ & $21.9 \pm 6.7$ & $18.9 \pm 5.5$ & $19.7 \pm 7.2$ & $16.9 \pm 6.2$ \\
\hline $1.9-2.3$ & $10.0 \pm 5.1$ & $17.3 \pm 4.9$ & $8.5 \pm 6.1$ & $18.2 \pm 5.8$ \\
\hline $0.6-2.3$ & $99.0 \pm 13.0$ & $102.0 \pm 12.0$ & $90.8 \pm 14.2$ & $100.2 \pm 13.4$ \\
\hline$B^{0}$ & $52.2 \pm 9.1$ & $57.6 \pm 9.1$ & $48.2 \pm 9.9$ & $56.4 \pm 9.9$ \\
\hline$B^{ \pm}\left(K^{ \pm}\right)$ & $37.0 \pm 7.4$ & $24.7 \pm 6.6$ & $33.1 \pm 8.2$ & $21.7 \pm 7.6$ \\
\hline$B^{ \pm}\left(K^{0}\right)$ & $10.1 \pm 4.8$ & $19.3 \pm 5.2$ & $9.9 \pm 5.2$ & $21.5 \pm 5.6$ \\
\hline \multicolumn{5}{|c|}{ Lepton-untagged events } \\
\hline $0.6-1.1$ & $392 \pm 30$ & $418 \pm 31$ & $378 \pm 31.6$ & $396 \pm 32.9$ \\
\hline $1.1-1.5$ & $167 \pm 21$ & $142 \pm 22$ & $162.1 \pm 21.8$ & $135.7 \pm 22.9$ \\
\hline $1.5-1.9$ & $144 \pm 18$ & $135 \pm 19$ & $139.1 \pm 19.4$ & $124.1 \pm 21.0$ \\
\hline $1.9-2.3$ & $122 \pm 26$ & $85 \pm 33$ & $101 \pm 29.1$ & $67 \pm 35.5$ \\
\hline $0.6-2.3$ & $835 \pm 51$ & $809 \pm 51$ & $787 \pm 54.4$ & $769 \pm 54.4$ \\
\hline$B^{0}$ & $476 \pm 34$ & $465 \pm 36$ & $455 \pm 36$ & $447 \pm 38$ \\
\hline$B^{ \pm}\left(K^{ \pm}\right)$ & $249 \pm 29$ & $162 \pm 28$ & $229 \pm 31$ & $148 \pm 30$ \\
\hline$B \pm\left(K^{0}\right)$ & $107 \pm 23$ & $175 \pm 23$ & $100 \pm 24$ & $166 \pm 25$ \\
\hline
\end{tabular}

Table 29: Data peaking yield and corresponding reconstructed number of events (after the Monte Carlo peaking background from Table 25 is subtracted) as a function of the hadronic mass bins. The quoted errors are just statistical errors.

\begin{tabular}{|l||c|c|}
\hline$M_{X s}(\mathrm{GeV})$ & $A_{C P \text { tag. }}$ & $A_{C P \text { untag. }}$ \\
\hline $0.6-1.1$ & $-0.02 \pm 0.15 \pm 0.03$ & $0.003 \pm 0.059 \pm 0.015$ \\
$1.1-1.5$ & $0.04 \pm 0.21 \pm 0.07$ & $0.108 \pm 0.108 \pm 0.018$ \\
$1.5-1.9$ & $0.09 \pm 0.26 \pm 0.08$ & $0.072 \pm 0.110 \pm 0.033$ \\
$1.9-2.3$ & $-0.34 \pm 0.34 \pm 0.09$ & $0.224 \pm 0.293 \pm 0.038$ \\
\hline $0.6-2.3$ & $-0.04 \pm 0.10 \pm 0.02$ & $0.025 \pm 0.050 \pm 0.015$ \\
\hline$B^{0}$ & $-0.08 \pm 0.14 \pm 0.03$ & $0.015 \pm 0.059 \pm 0.014$ \\
$B^{ \pm}\left(K^{ \pm}\right)$ & $0.20 \pm 0.20 \pm 0.05$ & $0.222 \pm 0.117 \pm 0.018$ \\
$B^{ \pm}\left(K^{0}\right)$ & $-0.34 \pm 0.25 \pm 0.06$ & $-0.201 \pm 0.135 \pm 0.026$ \\
\hline
\end{tabular}

Table 30: Measured final $A_{C P}$ in data as a function of the hadronic mass bins. 

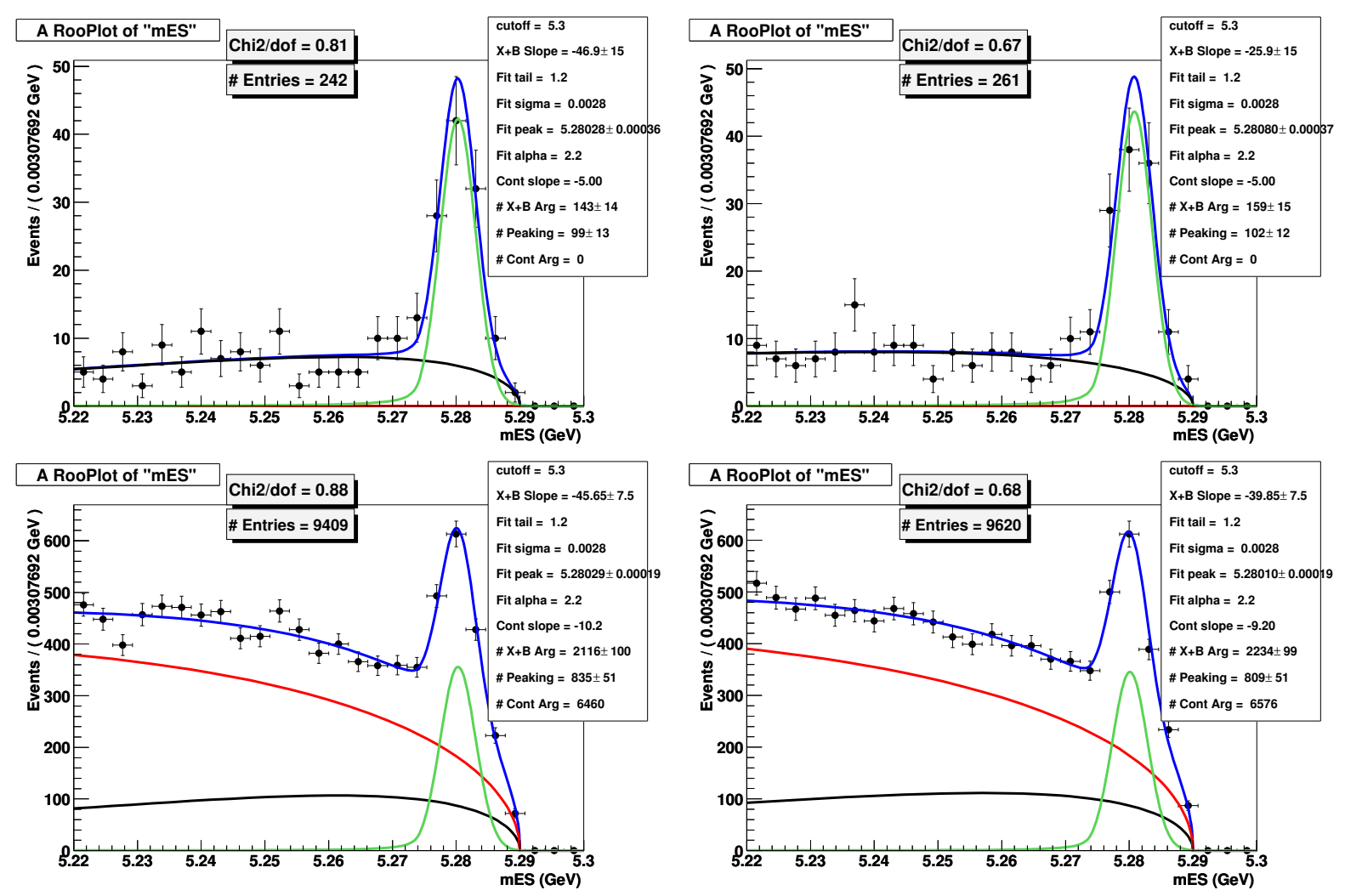

Figure 34: $m_{\mathrm{ES}}$ plot for all for data events $\left(M_{X s}=0.6-2.3 \mathrm{GeV}\right)$ : the first row shows the lepton-tagged events, the second row shows the lepton-untagged events. The left column shows the $b$ flavor sample, the left column shows the $\bar{b}$ flavor sample.

- The asymmetry is not seen in two hadronic body final states (non resonant $\mathrm{K} \pi$ decays).

- The asymmetry is seen in three and four hadronic body final states ( $\mathrm{K} \pi \pi$ and $\mathrm{K} \pi \pi \pi$ decays).

- The asymmetry is seen in the tagged sub-sample. Since the tagging is limited to the other $B$, this is almost an independent sample. 


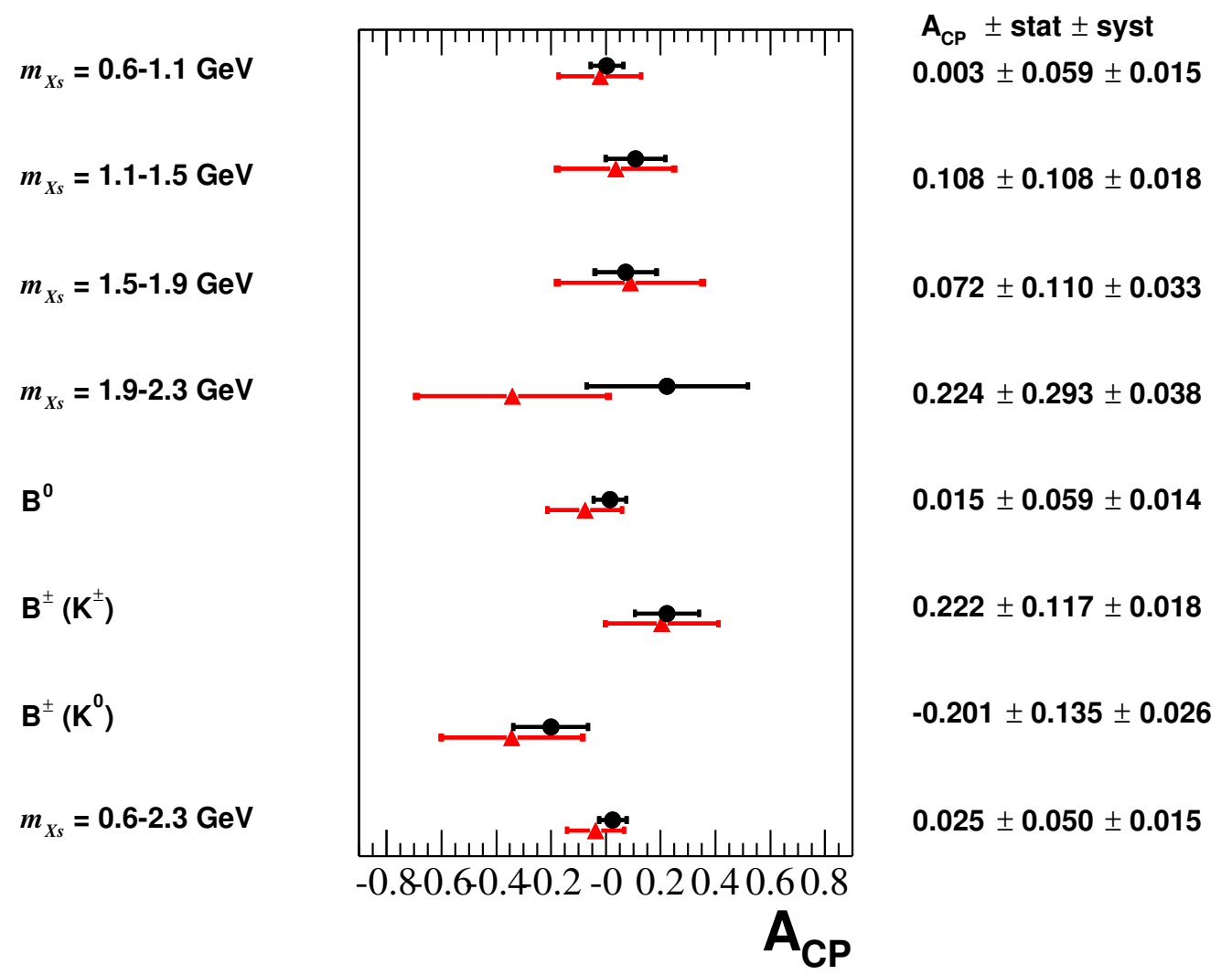

Figure 35: Measured $A_{C P}$ values for all the studied categories for lepton-tagged (triangles) and untagged (circles) events. The $A_{C P}$ numbers are for the untagged events. 
The further breakdown of the $C P$ asymmetry in the charged $B$ modes can be seen in Figures 36 and $37^{6}$.

We conclude that we are statistically limited and for now we interpret the split as a statistically fluctuation. More data will enlighten the reasons of the current $2.3 \sigma$ in the charged $B$ categories.

Finally, as shown in Table 30, the results obtained using lepton-tagged and untagged samples are very consistent, although the lepton-tagged sample corresponds only to a fraction of the total sample.

\footnotetext{
${ }^{6}$ As we do not have the breakdown of the mistag rates and detector shifts as a function of the decay mode and hadronic mass bins, which are limited by the signal statistics, the mistag rate and detector asymmetry as for the all category $M_{X s}=0.6-2.3 \mathrm{GeV}$ are used.
} 


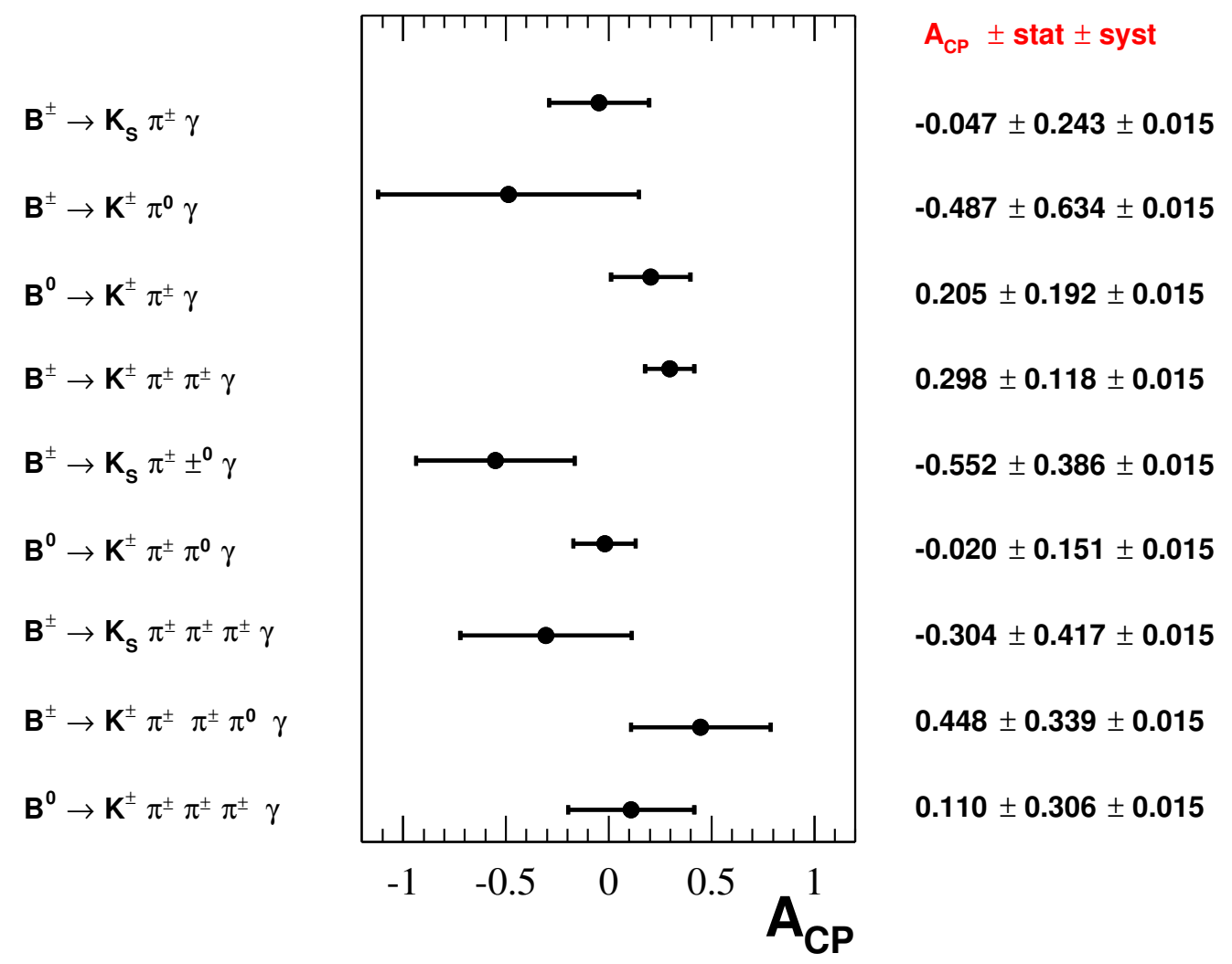

Figure 36: Measured direct $C P$ asymmetry as a function of decay mode. 


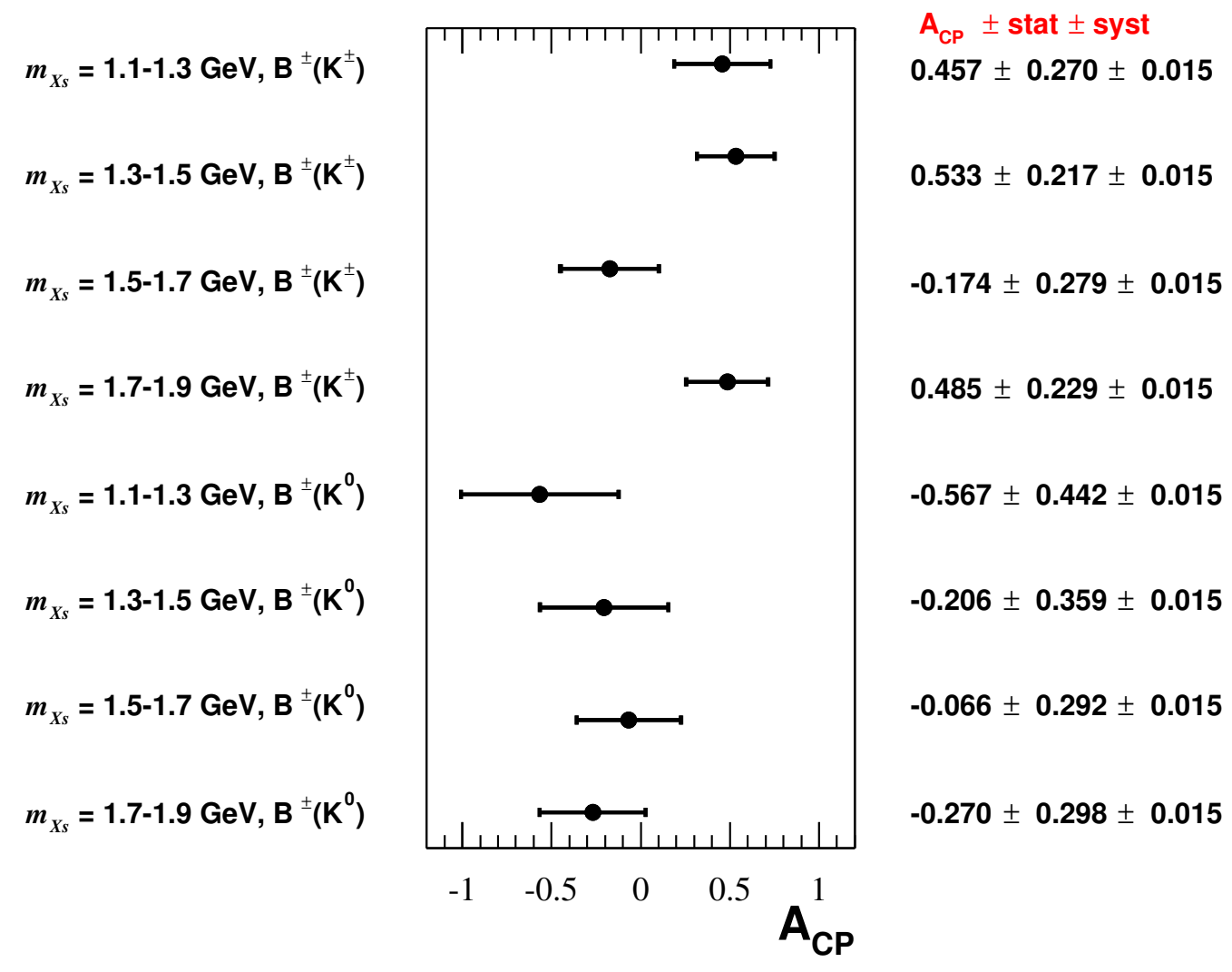

Figure 37: Measured direct $C P$ asymmetry as a function of hadronic mass. 


\section{Summary and Conclusions}

It looks like we have done a precision study.

The measurements of the direct $C P$ violation in the inclusive process $b \rightarrow s \gamma$ is performed using 89 million $B \bar{B}$ pairs. Signal event yields are extracted from the exclusive reconstruction of 12 final states, with continuum and $B \bar{B}$ backgrounds are reduced mainly by means of a neural network variable. The $B$ candidate with the smallest $\left|\Delta E^{*}\right|$ is chosen if more than one candidate survives the selection, and the $m_{\mathrm{ES}}$ distribution is fit to extract our signal. We perform separate analyses with and without a lepton-tag from the other $B$, since the leptontagging gives a significant reduction in continuum background. The flavor of the exclusive final states is identified either by the overall charge for $B^{ \pm}$decays, or by the charge of the Kaon in the $X_{s}$ system, for $B^{0}$ decays.

We split our sample into several categories for which the direct $C P$ asymmetry is measured. We show that the asymmetry does not depend on the hadronic mass, with the direct $C P$ asymmetry using all our reconstructed events is [38]:

$$
A_{C P}=0.025 \pm 0.050 \pm 0.015
$$

for the lepton-untagged sample, and

$$
A_{C P}^{\text {tag }}=-0.038 \pm 0.103 \pm 0.023
$$

for the lepton tagged sample. The results are consistent with Standard Model predictions within the experimental resolution. These results bound an $A_{C P}$ asymmetry in $B \rightarrow X_{s} \gamma$ decays to $-0.06<A_{C P}(b \rightarrow s \gamma)<+0.11$ at the $90 \%$ confidence level.

We can compare our results with the BELLE result of $A_{C P}$ in $B \rightarrow X_{S} \gamma$ decays [27]

introduced earlier. With a data sample of $140 \mathrm{fb}^{-1}$ they reconstruct 682 events to measure:

$$
A_{C P}^{B E L L E}=-0.004 \pm 0.051 \pm 0.038
$$


Both BELLE and BABAR $A_{C P}$ measurements are consistent with Standard Model predictions, but $C P$ asymmetries can arise from various Standard Model extensions. Various SUSY models (see Table 31 ) can increase $C P$ asymmetries to $\gtrsim 0.15$ in $B \rightarrow X_{S} \gamma$ decays, and with our current analysis we can only limit the possible $A_{C P}$ parameter phase space.

\begin{tabular}{|c|c|}
\hline Model & $A_{C P}$ \\
\hline Standard Model [25] & +0.004 \\
\hline 2 Higgs Doublet [39] & +0.006 \\
\hline 3 Higgs Doublet [39] & $\sim-0.03-+0.03$ \\
\hline Supergravity [40, 41, 42] & $\sim-0.10-+0.10$ \\
\hline SUSY with Squark Mixing [25, 43, 44] & $\sim-0.15-+0.15$ \\
\hline SUSY with R-parity Violation [45] & $\sim-0.17-+0.17$ \\
\hline
\end{tabular}

Table 31: $C P$ asymmetries in various models. Ranges are approximate using current knowledge of $B \rightarrow X_{s} \gamma$ decays.

\subsection{Outlook}

By 2006 BABAR should accumulate $\sim 500 \mathrm{fb}^{-1}$, and will improve our statistical errors by more than a factor of two. With the higher statistical sample we can also perform dedicated studies to determine detector $C P$ effects that will reduce the systematic error by greater than a factor of two.

With higher statistics, dedicated detector studies, and new signal extraction techniques, future $A_{C P}$ measurements of $B \rightarrow X_{S} \gamma$ decays should have total expected errors of $<0.02$. With these limits, we will be able to put stricter constraints on physics contributions beyond the Standard Model.

All work supported in part by the U.S. Department of Energy under contracts number DE-AC02-76SF00515 and DE-FG02-95ER40896. 


\section{A Truth-Match for Signal and Cross-Feed}

Truth-matching our signal events would mean finding a GHit associated track with the $B$ daughters and have the track come from the $B$ ("full truth-match"). In reality, we need to apply a "pseudo truth-match," for which we use the information carried on by the reconstructed hadronic mass, because the truth-matching is not perfect. This happens when one of the reconstructed daughters is not close enough to the MC truth track in $p / \theta / \phi$.

Our strategy is to allow for a window in reconstructed/true (i.e. generated) hadronic mass difference to catch the signal which would otherwise be lost. In Figure 38, the difference between the reconstructed $M_{X s}$ and the true $M_{X s}$ for "fully truth-matched" signal events is shown. This sample tells us which is our resolution on the hadronic mass.

Figure 39 shows the difference between the reconstructed $M_{X s}$ and the true $M_{X s}$ for events which are not "fully truth-matched," but whose tracks have an associated GHit track and the $B$ reconstructed final state is the same than the generated one. Both events with $M_{X s}$ greater and lower (ie $K^{*}$ mass peak) than $1.1 \mathrm{GeV}$ are shown. We can see that there is a combination of signal and Xfeed. The cut at $\mid$ Reco $M_{X s^{-}}$True $M_{X s} \mid<0.05 \mathrm{GeV}$ recovers these lost signal events. Actually at lower $M_{X s}$ masses, the window does not need to be as large due to the lower number of bodies. So for the $K^{*}$ region $\left(M_{X s}<1.1 \mathrm{GeV}\right)$ we only require this window to be $0.025 \mathrm{GeV}$. The events which do not pass the cut on the hadronic mass are considered cross-feed events.

Figure 40 shows all the other events, that is, the ones whose tracks have all a GHit track associated but the reconstructed final state is different from the generated one, and all the events in which at least a $B$ daughter track does not have an associated GHit track. 


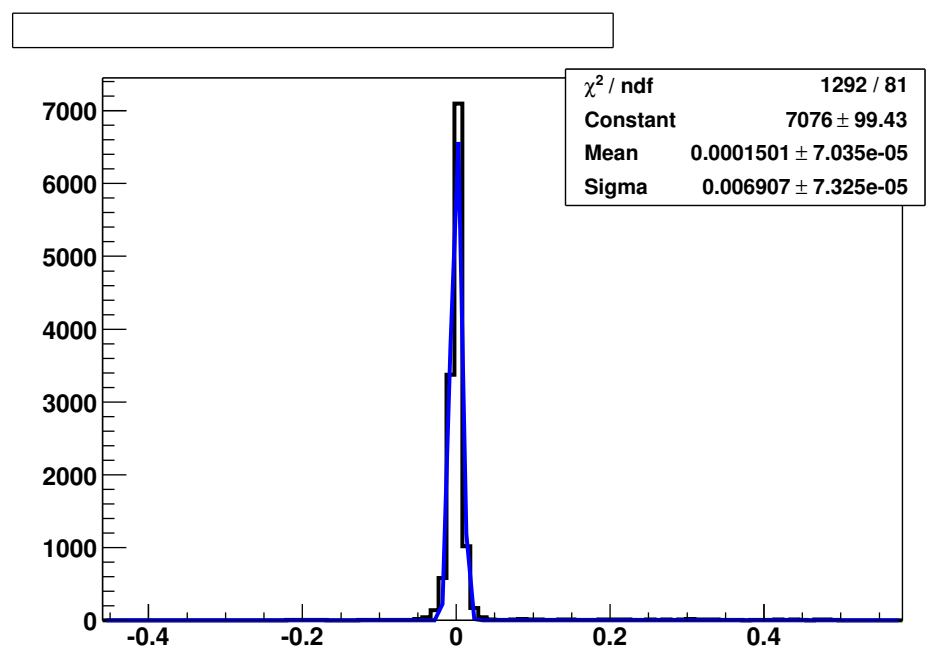

Figure 38: (Reco $M_{X s^{-}}$True $\left.M_{X s}\right)(\mathrm{GeV})$ for fully truth-matched signal events.


Figure 39: (Reco $M_{X s^{-}}$True $\left.M_{X s}\right)(\mathrm{GeV})$ for events which are not "fully truth-matched," but whose tracks have an associated GHit track and the $B$ reconstructed final state is the same than the generated one. The left plots shows the events for $M_{X s}>1.1$ and the right one for $M_{X s}<1.1$. 


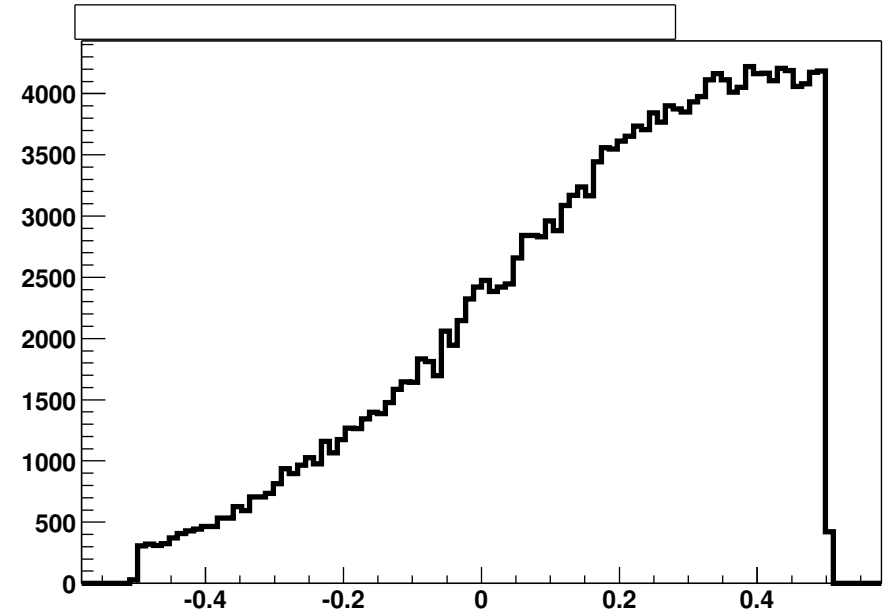

Figure 40: (Reco $M_{X s^{-}}$True $\left.M_{X s}\right)(\mathrm{GeV})$ for Xfeed events: events whose tracks have all a GHit track associated but the reconstructed final state is different from the generated one, and all the events in which at least a $B$ daughter track does not have an associated GHit track. 


\section{B The PDFs used in the fits}

\section{B.1 Argus Background Distribution}

The Argus distribution for the background shape defined:

$$
A\left(m ; m_{0}, c\right)=\frac{1}{N} \cdot m \sqrt{1-\left(m / m_{0}\right)^{2}} \cdot \exp \left(c\left(1-\left(m / m_{0}\right)^{2}\right)\right) \quad\left(m<m_{0}\right) .
$$

Note that $m_{0}$ represents the kinematic upper limit for the constrained mass and is usually held fixed at half of the center of mass energy (nominally $5.29 \mathrm{GeV} / c^{2}$ for $\mathrm{Y}(4 \mathrm{~S})$ events) in a fit. The minimum value of $m_{0}$ must be $\geq$ the maximum value of $m$. The parameter $c$ is what is usually referred to as the Argus parameter or Argus shape parameter in the text.

\section{B.2 Crystal Ball Line Shape Distribution}

The Crystal Ball line shape distribution:

$$
C\left(m ; m_{0}, \sigma, \alpha, n\right)=\frac{1}{N} \cdot \begin{cases}\exp \left(-\left(m-m_{0}\right)^{2} /\left(2 \sigma^{2}\right)\right) & , m>m_{0}-\alpha \sigma \\ \frac{(n / \alpha)^{n} \exp \left(-\alpha^{2} / 2\right)}{\left(\left(m_{0}-m\right) / \sigma+n / \alpha-\alpha\right)^{n}} & , m \leq m_{0}-\alpha \sigma\end{cases}
$$

is useful for fitting a radiative tail. It consists of a Gaussian signal peak matched to a power law tail. Note that the tail parameter, $n$, is not necessarily an integer, and is usually held fixed in a fit: lower values generate a longer tail. The parameter $\alpha$ determines the crossover point from the Gaussian distribution to the power law tail distribution, in units of the peak width, $\sigma$. Typical values for $|\alpha|$ are $0.6-1.1$. With $\alpha>0$ the tail is below the peak, and with a negative value and the two ranges switched the tail is above the peak. 


\section{Toy Study}

To ensure that the fitting is done correctly, we run a toy Monte Carlo study. The toy Monte Carlo example are not for $b$ or $\bar{b}$ specific events, and were from an earlier data set without optimized cuts. The toy set simply gives us a range of signal to background ratios to check our fit technique.

First, we have checked that if the cross-feed and generic $B \bar{B}$ are added together, and then fit with a single Crystal Ball and Argus function, we get the same number of peaking background events in the Crystal Ball function as we do if we fit them separately. Examples of the expected numbers of peaking events from several fits can be found in Table 32. In the table we report the results from the fits to the Xfeed and $B \bar{B}$ samples separately, the Xfeed and $B \bar{B}$ together, the Xfeed, $B \bar{B}$ and continuum together, the full Monte Carlo (i.e. backgrounds and signal events summed up). The difference between the number of events from the full Monte Carlo fit and the peaking component of the fit to the Xfeed, $B \bar{B}$ and continuum together is our signal background subtracted, to be compared to the expected signal events.

Second, we check whether the number of peaking background and signal events has any bias. Table 33 gives the percent of the pulls and the statistical error in percent for our toy Monte Carlo examples. Note that the statistical error is much larger than the pull error. The largest expected pull relative to expected statistical error is less than $4 \%$. This gives an expected pull of $<1$ event for the worst case scenario. The statistical errors of the full fits are much larger that one event, so we conclude that the fit method does not contribute a significant systematic error. 


\begin{tabular}{|c|c|c|c|c|c|}
\hline $\begin{array}{c}M_{X s} \\
(\mathrm{GeV})\end{array}$ & $\begin{array}{c}\text { Peaking } \\
\text { Xfeed }\end{array}$ & $\begin{array}{c}\text { Peaking } \\
B \bar{B}\end{array}$ & $\begin{array}{c}\text { Peaking Combined } \\
\text { Xfeed and } B \bar{B}\end{array}$ & $\begin{array}{c}\text { Peaking } \\
\text { Full Fit }\end{array}$ & $\begin{array}{c}\text { Expected } \\
\text { Signal }\end{array}$ \\
\hline $1.1-1.2$ & $1.2 \pm 2.3$ & $0.0 \pm 0.4$ & $0.7 \pm 2.3$ & $60 \pm 10$ & 65.1 \\
$1.2-1.4$ & $2.4 \pm 5.4$ & $0.0 \pm 3.3$ & $2.4 \pm 4.3$ & $137 \pm 15$ & 133.6 \\
$1.4-1.6$ & $6.2 \pm 5.8$ & $1.0 \pm 2.2$ & $7.3 \pm 5.8$ & $105 \pm 23$ & 106.0 \\
$1.6-1.8$ & $5.1 \pm 7.9$ & $0.0 \pm 5.7$ & $5.0 \pm 6.2$ & $76 \pm 14$ & 73.7 \\
$1.8-2.0$ & $8.1 \pm 7.4$ & $4.0 \pm 4.9$ & $8.4 \pm 7.3$ & $51 \pm 14$ & 54.9 \\
$2.0-2.2$ & $1.7 \pm 8.1$ & $1.1 \pm 6.4$ & $1.8 \pm 6.1$ & $32 \pm 11$ & 19.6 \\
$2.2-2.4$ & $2.3 \pm 7.4$ & $2.3 \pm 7.4$ & $2.0 \pm 7.5$ & $20 \pm 12$ & 10.5 \\
\hline
\end{tabular}

Table 32: This table shows results from toy data set fits to the peaking components of Monte Carlo samples, number of expected peaking background from cross-feed and $B \bar{B}$ Monte Carlo, their combined fit, fit after adding the continuum Monte Carlo, and then fit after adding the signal Monte Carlo. One can subtract the "Peaking Combined Xfeed + $B \bar{B}$ " from the "Peaking Full Fit" column to get the result in the "Signal" column. These numbers come from the toy study data set, and are to be used to show that the fitting technique is consistent.

\begin{tabular}{|c|c|c|c|c|c|c|}
\hline $\begin{array}{c}M_{X s} \\
(\mathrm{GeV})\end{array}$ & $\begin{array}{c}\text { Pull in } \\
\text { Peaking BG (\%) }\end{array}$ & $\begin{array}{c}\text { Stat. Error in } \\
\text { Peaking BG (\%) }\end{array}$ & $\begin{array}{c}\text { Pull in } \\
\text { Full BG (\%) }\end{array}$ & $\begin{array}{c}\text { Stat. Error in } \\
\text { Full BG (\%) }\end{array}$ & $\begin{array}{c}\text { Pull in } \\
\text { Sig.+BG (\%) }\end{array}$ & $\begin{array}{c}\text { Stat. Error in } \\
\text { Sig.+B }(\%)\end{array}$ \\
\hline $1.1-1.2$ & 9 & 329 & 8 & 111 & 1 & 52 \\
$1.2-1.4$ & 12 & 179 & 12 & 214 & 2 & 36 \\
$1.4-1.6$ & 12 & 79 & 13 & 506 & 1 & 22 \\
$1.6-1.8$ & 9 & 124 & 31 & 783 & 3 & 18 \\
$1.8-2.0$ & 6 & 87 & 18 & 81 & 4 & 12 \\
$2.0-2.2$ & 7 & 66 & 10 & 275 & 2 & 12 \\
$2.2-2.4$ & 2 & 375 & & 12 & 2 & 9 \\
\hline
\end{tabular}

Table 33: The pull error and statistical error for all the toy examples. Large statistical errors come from low statistics, e.g. for the 1.6-1.8 GeV bin, the $783 \%$ statistical error comes from an expected peaking component of $1.2 \pm 9.4$ events. These numbers come from the toy study data set, and are to be used to show that the fitting technique is consistent. All possible pulls correspond to pulls of $<1$ event in the final fit, and are not consistently in the positive or negative yield direction. 


\section{Supplementary Systematics Section}

\section{D.1 CP Asymmetry in Mistagged Signal Monte Carlo Events due to Mis-ID Biases}

Some signal events are not properly tagged in the correct $C P$ state due to detector effects. This can generate a fake $C P$ asymmetry. In this section we discuss only those events that are still classified as "true" signal events, i.e. reconstructed in the same or opposite flavor, and with a hadronic mass within $50 \mathrm{MeV}$ of the generated value. Events reconstructed with opposite flavor are what we call "mistagged" events.

Table 34 shows how signal events can have mis-identified particles. This can lead to a different or identical $C P$ to the one with which they were generated. In the table we ignore cases in which the mis-ID is due to the combination of several sources as this gives a negligible contribution. There is a negligible contribution to this from charge confusion, (i.e. change of the charge(s) of a $K^{+}$and/or a $\pi^{-}$), but there is a higher contribution from pion mis-identification as a kaon ${ }^{7}$.

We estimate the mistag rate using reconstructed signal Monte Carlo event. However, the Monte Carlo does not reproduce correctly the asymmetry in data events; we have reweighted the Monte Carlo events as described in Section 9.1, and we apply the tracking and PID tables (pion to Kaon mis-identification rate as a function of the pion momentum from the PID tables is shown in Table 43). The mistag rate is defined as the percentage of times the wrong $b$-flavor is reconstructed with respect to all the events which pass the selection. Results are reported in Table 35 as a function of the hadronic mass. The error on the mistag rate is the sum in quadrature of the statistical error, and the error from the variation of of the weights $\pm 1 \sigma$ from Tables 22 and 23. Note that in case that there are not enough

\footnotetext{
${ }^{7}$ Note that we may easily have a kaon mis-identified as a pion $\left(K^{+} \rightarrow \pi^{+}\right)$, since there is no particle identification applied to the charged pions (i.e. we do not veto charged Kaons in the pion selection).
} 
statistics to measure the mistag rate, so we assume that the mistag rate is zero.

In order to estimate the flavor asymmetry in the misidentified events, we compute the asymmetry of the wrongly reconstructed $b$ flavor events versus the wrongly reconstructed $\bar{b}$ events, normalized by the total events which pass the selection. The results are shown in Table 36, and the asymmetry is consistent with zero within one sigma. The detector asymmetry and the mistag rate error from the flavor asymmetry from misidentified events is: the sum in quadrature of the statistical error and the error from the variation of of the weights $\pm 1 \sigma$ from Tables 22 and 23 . Note that in case that there are not enough statistics to measure the flavor asymmetry in the misidentified events, we assume that the flavor asymmetry is zero.

\begin{tabular}{|l|c|c|}
\hline Final State & Same flavor & Opposite flavor \\
\hline$B^{+} \rightarrow K_{s} \pi^{+} \gamma$ & - & $\pi^{+} \rightarrow \pi^{-}$ \\
$B^{+} \rightarrow K^{+} \pi^{0} \gamma$ & - & $K^{+} \rightarrow K^{-}$ \\
$B^{0} \rightarrow K^{+} \pi^{-} \gamma$ & - & $K^{+} \pi^{-} \rightarrow \pi^{+} K^{-}$ \\
$B^{+} \rightarrow K^{+} \pi^{+} \pi^{-} \gamma$ & $K^{+} \pi^{+} \rightarrow \pi^{+} K^{+}$ & $K^{+} \rightarrow K^{-}$ \\
$B^{+} \rightarrow K_{s} \pi^{+} \pi^{0} \gamma$ & - & $\pi^{+} \rightarrow \pi^{-}$ \\
$B^{+} \rightarrow K^{+} \pi^{0} \pi^{0} \gamma$ & - & $K^{+} \rightarrow K^{-}$ \\
$B^{0} \rightarrow K^{+} \pi^{-} \pi^{0} \gamma$ & - & $K^{+} \pi^{-} \rightarrow \pi^{+} K^{-}$ \\
$B^{+} \rightarrow K_{s} \pi^{+} \pi^{-} \pi^{+} \gamma$ & - & $\pi^{+} \rightarrow \pi^{-}$ \\
$B^{+} \rightarrow K^{+} \pi^{+} \pi^{-} \pi^{0} \gamma$ & $K^{+} \pi^{+} \rightarrow \pi^{+} K^{+}$ & $K^{+} \rightarrow K^{-}$ \\
$B^{+} \rightarrow K_{s} \pi^{+} \pi^{0} \pi^{0} \gamma$ & - & $\pi^{-} \rightarrow \pi^{+}$ \\
$B^{0} \rightarrow K^{+} \pi^{-} \pi^{+} \pi^{-} \gamma$ & $K^{+} \pi^{+} \rightarrow \pi^{+} K^{+}$ & $K^{+} \pi^{-} \rightarrow \pi^{+} K^{-}$ \\
$B^{0} \rightarrow K^{+} \pi^{-} \pi^{0} \pi^{0} \gamma$ & - & $K^{+} \pi^{-} \rightarrow \pi^{+} K^{-}$ \\
\hline
\end{tabular}

Table 34: Cases in which mistagged signal events generate the same or the opposite flavor due to charge confusion or pion mis-ID as a Kaon. It is assumed that the signal events are not re-classified as cross-feed as a result of the mis-interpretation. 


\begin{tabular}{|l|c|c|}
\hline \multicolumn{3}{|c|}{ mistag rate for signal events } \\
$M_{X s}(\mathrm{GeV})$ & $\omega_{\text {tag }}$ & $\omega_{\text {untag }}$ \\
\hline \hline \multicolumn{3}{|c|}{$b$ flavor } \\
\hline $0.6-1.1$ & $0.0017 \pm 0.0009$ & $0.0049 \pm 0.0005$ \\
$1.1-1.5$ & $0.013 \pm 0.007$ & $0.012 \pm 0.003$ \\
$1.5-1.9$ & - & $0.004 \pm 0.002$ \\
$1.9-2.3$ & $0.015 \pm 0.011$ & $0.014 \pm 0.004$ \\
\hline $0.6-2.3$ & $0.0021 \pm 0.0009$ & $0.0052 \pm 0.0005$ \\
\hline$B^{0}$ & $0.004 \pm 0.002$ & $0.0073 \pm 0.0008$ \\
$B^{ \pm}\left(K^{ \pm}\right)$ & - & $0.0014 \pm 0.0005$ \\
$B \pm\left(K^{0}\right)$ & $0.006 \pm 0.003$ & $0.006 \pm 0.001$ \\
\hline \hline \multicolumn{3}{|c|}{$\bar{b}$ flavor } \\
\hline $0.6-1.1$ & $0.006 \pm 0.002$ & $0.0052 \pm 0.0008$ \\
$1.1-1.5$ & $0.014 \pm 0.008$ & $0.008 \pm 0.003$ \\
$1.5-1.9$ & $0.012 \pm 0.0009$ & $0.007 \pm 0.004$ \\
$1.9-2.3$ & - & $0.010 \pm 0.005$ \\
\hline $0.6-2.3$ & $0.007 \pm 0.002$ & $0.0056 \pm 0.0007$ \\
\hline$B^{0}$ & $0.012 \pm 0.003$ & $0.0072 \pm 0.0011$ \\
$B \pm\left(K^{ \pm}\right)$ & - & $0.0009 \pm 0.0006$ \\
$B \pm\left(K^{0}\right)$ & $0.002 \pm 0.002$ & $0.007 \pm 0.002$ \\
\hline
\end{tabular}

Table 35: The mistag rate for signal events lepton-tagged $\left(\omega_{\text {tag }}\right)$ and untagged $\left(\omega_{\text {untag }}\right)$ events is shown for the $b$ and $\bar{b}$ flavors. Monte Carlo events are re-weighted. Where there is a "-," means that there were not enough statistics.

\begin{tabular}{|l||c|c|}
\hline$M_{X s}(\mathrm{GeV})$ & $A_{C P}^{\text {fake }}{ }_{\text {tag. }}$ & $A_{C P}^{\text {fake }}$ untag. \\
\hline $0.6-1.1$ & $-0.002 \pm 0.001$ & $-0.0002 \pm 0.0004$ \\
$1.1-1.5$ & $0.000 \pm 0.006$ & $0.002 \pm 0.002$ \\
$1.5-1.9$ & $-0.006 \pm 0.005$ & $-0.002 \pm 0.002$ \\
$1.9-2.3$ & $0.008 \pm 0.006$ & $0.002 \pm 0.004$ \\
\hline $0.6-2.3$ & $-0.002 \pm 0.001$ & $-0.0001 \pm 0.0005$ \\
\hline$B^{0}$ & $-0.004 \pm 0.002$ & $0.000 \pm 0.0008$ \\
$B^{ \pm}\left(K^{ \pm}\right)$ & - & $0.0003 \pm 0.0005$ \\
$B^{ \pm}\left(K^{0}\right)$ & $0.002 \pm 0.002$ & $-0.0005 \pm 0.0012$ \\
\hline
\end{tabular}

Table 36: Fake $A_{C P}$ in mistagged events due to detector bias as a function of the hadronic mass bins. The events are split into lepton-tagged $\left(A_{C P}{ }_{\text {fake }}{ }_{\text {tag. }}\right)$ and untagged events $\left(A_{C P}^{\text {fake }}{ }_{\text {untag. }}\right)$. Monte Carlo events are re-weighted. Where there is a "-," means that there were not enough statistics. 


\section{D.2 CP Asymmetry in Cross-Feed Monte Carlo Events Due to Detector Effects}

There can also be a $C P$ asymmetry in the cross-feed events due to detector efficiency bi-

ases. In this section we consider only the peaking component of the cross-feed which is subtracted directly from the fitted yields in data. Cross-feed includes signal events which are reconstructed in a different final state from which they were generated, either due to the replacement of one particle by another (e.g. $K_{S}$ replaced by a charged kaon or $\pi^{0}$ replaced by a charged track), or due to the event being reconstructed with more or less particles than the generated final state. Cross-feed also includes cases where the final state is the same as from which they were generated (although it may have the opposite flavor), but the reconstructed hadronic mass differs from the generated mass by more than $50 \mathrm{MeV}$. Finally, the cross-feed includes the other $b \rightarrow s \gamma$ final states which were not included in our study.

Table 44 identifies the different cases in which replacing a particle leads to a final state different from the signal final state. Table 45 shows the cases of adding or removing a particle. Note that we have to include the cases where an initial mixed-flavor becomes a $b$ or $\bar{b}$ flavor (is included in our 12 modes), and where an initial $b$ or $\bar{b}$ flavor becomes a mixed-flavor (is removed from the 12 modes).

There are no recipes available for estimating the rates for replacing or adding particles, so we use the peaking component of the cross-feed events from the reconstructed signal Monte Carlo samples. Results of the difference in the peaking component of $b$ and $\bar{b}$ crossfeed events are shown in Table 37. As the statistical error is very large we do not observe any asymmetry. We do not reweight the cross-feed events as we do for the signal, to take into account detector asymmetries, because the statistical error is so large that variations of $1-2 \%$ are negligible. We note that the peaking background components are just $\leq 5 \%$ of the peaking yield. 


\begin{tabular}{|c|c|c|c|c|c|c|}
\hline \multirow[b]{2}{*}{$M_{X s}(\mathrm{GeV})$} & \multicolumn{6}{|c|}{ Cross-feed events } \\
\hline & $b$ & $\bar{b}$ & $A_{C P \text { taq. }}^{X \text { feed }}$ & $b$ & $\bar{b}$ & $A_{C P \text { untag. }}$ \\
\hline $0.6-1.1$ & $3.85 \pm 3.10$ & $1.30 \pm 3.73$ & $0.50 \pm 0.97$ & $13.4 \pm 10.0$ & $22.3 \pm 10.6$ & $-0.25 \pm 0.43$ \\
\hline $1.1-1.5$ & $0 \pm 49.2$ & $10.0 \pm 50.2$ & $0.00 \pm 03.51$ & $3.77 \pm$ & $5.22 \pm 6.08$ & 0.95 \\
\hline $1.5-1.9$ & $39 \pm 2.29$ & $1.0 \pm 2.29$ & $16 \pm 1$ & $3.77 \pm 7.36$ & $6.72 \pm 7.57$ & $-0.28 \pm 1.05$ \\
\hline $1.9-2.3$ & $.44 \pm 2.22$ & $0.38 \pm 1.60$ & $58 \pm 1.96$ & $4.58 \pm 6.65$ & $-0.015 \pm 6.36$ & $1.01 \pm 2.92$ \\
\hline $0.6-2.3$ & $30.5 \pm 14.6$ & $2.77 \pm 4.32$ & $0.83 \pm 0.80$ & $30.5 \pm 14.6$ & $32.2 \pm 14.9$ & $-0.03 \pm 0.33$ \\
\hline$B^{0}$ & $2.86 \pm 2.95$ & $0.31 \pm 2.46$ & 1.69 & $15.29 \pm 9.95$ & $10.43 \pm 9.99$ & 0.56 \\
\hline$B^{ \pm}$ & $3.02 \pm 3.40$ & $2.05 \pm 2.75$ & $0.19 \pm 0.91$ & $10.03 \pm 8.94$ & $11.91 \pm 8.86$ & $-0.09 \pm 0.58$ \\
\hline$B^{ \pm}\left(K^{0}\right)$ & $0.75 \pm 1.61$ & $0.30 \pm 2.05$ & $0.43 \pm 2.46$ & $5.00 \pm 6.58$ & $9.71 \pm 7.36$ & $-0.32 \pm 0.73$ \\
\hline
\end{tabular}

Table 37: Fit peaking component for $b$ and $\bar{b}$ cross-feed Monte Carlo events and corresponding $A_{C P}$ for lepton-tagged and untagged events. The events are scaled to luminosity.

\section{D.3 Background Asymmetry of Monte Carlo Events}

Similarly to signal events, background events are affected by the detector asymmetry. In this section we study the apparent $C P$ asymmetry in Monte Carlo background events. Note that the fits (described in Section 7) assume that the $b$ and $\bar{b}$ flavors have the same fitting parameters. This assumption is cross-checked in Section D.4.

To study the Monte Carlo background asymmetry we look at the generic $B \bar{B}$ Monte Carlo events and the continuum Monte Carlo events. We compare the number of events in the fitted Argus shape and the peaking Crystal Ball for $b$ and $\bar{b}$ events. Only lepton-untagged samples are used because of higher statistics. The results are shown in Table 38. As in the signal Monte Carlo events, we do not see any asymmetry in the background Monte Carlo events.

\section{D.4 Variation of Fitting Parameters}

In performing the fits described in Section 7, the Crystal Ball and one of the two Argus functions are fixed (see Table 15). The Crystal Ball parameters are obtained from a weighted average of the parameters from the fit to the $b+\bar{b}$ signal events in the hadronic mass bins (see Table 16). The fixed Argus shape parameters come from the fit to the continuum Monte 


\begin{tabular}{|c|c|c|c|c|c|c|}
\hline \multirow[b]{2}{*}{$M_{X s}(\mathrm{GeV})$} & \multicolumn{3}{|c|}{ Argus } & \multicolumn{3}{|c|}{ Crystal Ball } \\
\hline & $\bar{b}$ & $b$ & $A_{C P}$ & $\bar{b}$ & $b$ & $A_{C P}$ \\
\hline & \multicolumn{6}{|c|}{ Generic $B \bar{B}$ MC } \\
\hline $0.6-1.1$ & $17.7 \pm 4.9$ & $12.3 \pm 4.4$ & $-0.18 \pm 0.22$ & $-0.7 \pm 2.4$ & $.7 \pm 2.8$ & $0.01 \pm 2.2$ \\
\hline $1.1-1.5$ & $19.4 \pm 4.8$ & $20.2 \pm 4.8$ & $0.02 \pm 0.18$ & $0.6 \pm 2.0$ & $0.1 \pm 1.7$ & $-0.71 \pm 4.9$ \\
\hline $1.5-1.9$ & $106 \pm 11$ & $95 \pm 10$ & $-0.05 \pm 0.07$ & $2.9 \pm 4.8$ & $8.0 \pm 2.8$ & $0.47 \pm 0.44$ \\
\hline $1.9-2.3$ & $493 \pm 24$ & $490 \pm 24$ & $0.00 \pm 0.03$ & $1.9 \pm 10.2$ & $14.9 \pm 10.6$ & $0.77 \pm 1.12$ \\
\hline $0.6-2.3$ & $636 \pm 28$ & $641 \pm 28$ & $0.004 \pm 0.31$ & $5.1 \pm 11.6$ & $13.9 \pm 11.8$ & $0.46 \pm 0.96$ \\
\hline$B^{0}$ & $284 \pm 19$ & $281 \pm 19$ & $-0.01 \pm 0.05$ & $6.3 \pm 8.6$ & $4.6 \pm 8.3$ & $-0.16 \pm 1.12$ \\
\hline$B^{ \pm}\left(K^{ \pm}\right)$ & $214 \pm 16$ & $224 \pm 16$ & $0.02 \pm 0.05$ & $0.0 \pm 6.1$ & $7.6 \pm 6.7$ & $0.33 \pm 0.43$ \\
\hline \multirow[t]{2}{*}{$B^{ \pm}\left(K^{0}\right)$} & $137 \pm 13$ & $137 \pm 13$ & $0.00 \pm 0.07$ & $-0.9 \pm 4.9$ & $1.2 \pm 5.2$ & $0.91 \pm 4.33$ \\
\hline & \multicolumn{6}{|c|}{ Continuum MC } \\
\hline $0.6-1.1$ & 1261 & 1245 & $-0.01 \pm 0.02$ & & & \\
\hline $1.1-1.5$ & 848 & 842 & $0.00 \pm 0.02$ & & & \\
\hline $1.5-1.9$ & 1570 & 1531 & $-0.01 \pm 0.02$ & & & \\
\hline $1.9-2.3$ & 2879 & 2842 & $-0.01 \pm 0.01$ & & & \\
\hline $0.6-2.3$ & 6576 & 6460 & $-0.009 \pm 0.009$ & & & \\
\hline$B^{0}$ & 2584 & 2548 & $-0.01 \pm 0.01$ & & & \\
\hline$B^{ \pm}\left(K^{ \pm}\right)$ & 2491 & 2369 & $-0.02 \pm 0.01$ & & & \\
\hline$B^{ \pm}\left(K^{0}\right)$ & 1573 & 1543 & $-0.01 \pm 0.02$ & & & \\
\hline
\end{tabular}

Table 38: Fitted number of events in Argus and peaking the Crystal Ball fitted distributions in generic $B \bar{B}$ and continuum Monte Carlo events for $b$ and $\bar{b}$ flavors. The corresponding $C P$ asymmetry is computed. The events are lepton-untagged. 
Carlo.

In this section, we cross-check that the fitting parameters are identical between the $b$ and $\bar{b}$ flavors. We have looked at the lepton-untagged sample as there are more statistics. We have made the following tests:

- We have looked at the fits on the continuum Monte Carlo and checked that the Argus shape parameter is the same for $b$ and $\bar{b}$ final states (see Table 46 ).

- We have fit the signal events with a free Crystal Ball shape and checked that the Argus shape parameter is the same for $b$ and $\bar{b}$ final states (see Table 47 ).

- We have looked at the fits on Xfeed $+B \bar{B}$ background and checked that the Argus shape parameter is the same for $b$ and $\bar{b}$ final states (see Table 48 ).

The results are practically identical between the $b$ and $\bar{b}$ final states, as expected. Thus, we do not attribute any systematic error for possible differences in the fitting parameters.

\section{D.5 NN Validation}

We validate the NN showing that there is a good agreement between data and Monte Carlo for both the background and signal events. In order to compare the NN for background events, we have used the $9.6 \mathrm{fb}^{-1}$ of off-resonance data. The overlay of the off-resonance data and continuum Monte Carlo (normalized to the same luminosity) is shown in Figure 41. Similarly, we look at the NN for the signal $B \rightarrow K^{*} \gamma$ data (see section 8) and Monte Carlo events in Figure 42 for lepton-tagged and untagged events.

\section{D.6 Fragmentation Corrections}

The Monte Carlo generator for inclusive $B \rightarrow X_{S} \gamma$ uses JETSET to fragment the $X_{S}$ system, ignoring resonant substructure. The fractions of each final state obtained from JETSET are 

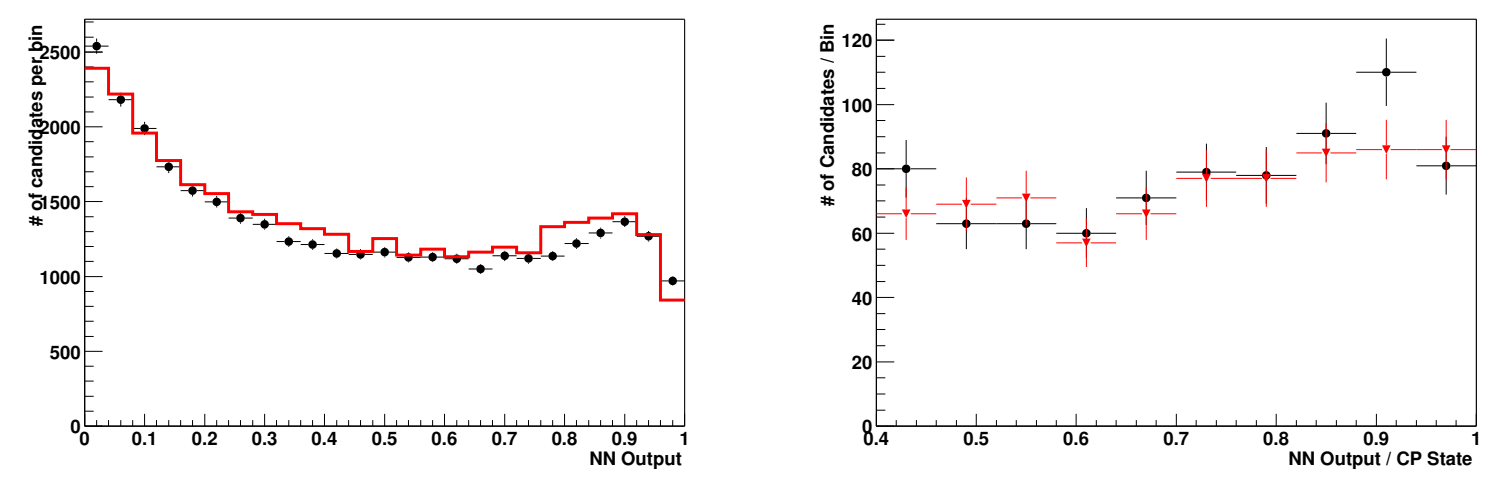

Figure 41: The left plot is an overlay of $9.6 \mathrm{fb}^{-1}$ of off-resonance data on top of an equal amount of $u d s$ and $c \bar{c}$ Monte Carlo. The right plot is of the off-resonance NN output (after all cuts and $\left|\Delta E^{*}\right|$ best candidate selection) for the $\bar{b}$ (circles) and $b$ (triangles) final states.
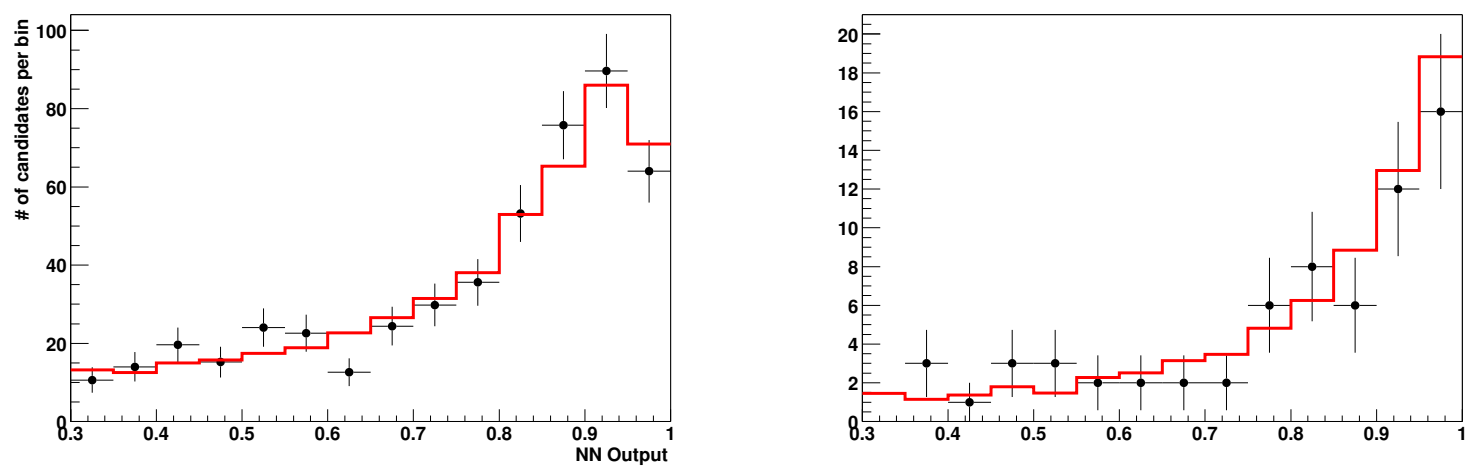

Figure 42: The plots (left untagged background subtracted, right tagged) are an overlay of scaled $B \rightarrow K^{*} \gamma$ Monte Carlo signal, and on resonance data after all the selection cuts are applied. Moreover, the $m_{\mathrm{ES}}$ cut has been tightened to $5.271 \mathrm{GeV}$ to show the NN performance on "signal" like events. 
not expected to model the data accurately due to this unmodeled resonance structure. To check this, we compare data and Monte Carlo fractions above $M_{X s}=1.1 \mathrm{GeV}$ in the various categories. In particular, the dominant contribution will be given by the ratio of charged and neutral kaons. When applying the fragmentation corrections, we re-weight both the signal and cross-feed events according to the data Monte Carlo comparison.

We first try a "worst case" scenario by reweighting the Monte Carlo $K^{ \pm}$to $K_{S}$ ratio. We increase the $K^{ \pm}$relative yields by $10 \%$ in signal Monte Carlo events. We refit the peaking background events and the full Monte Carlo events, and from the difference of the peaking components we compute the $A_{C P}$. The results can be found in Table 39 and show no evidence of an induced $A_{C P}$. We have also done the same study with the $\pi^{0}$ to $\pi$ ratio set to 1.5 . This $50 \%$ deviation gives a $C P$ asymmetry of $0.05 \pm 0.07$.

\begin{tabular}{|l||c|c||c|c||c||}
\hline \multicolumn{1}{|c||}{} & \# Peaking Background & \# Peaking Signal + Background & \\
$M_{X s}(\mathrm{GeV})$ & $b$ & $\bar{b}$ & $b$ & $\bar{b}$ & $A_{C P}$ \\
\hline \hline \multicolumn{7}{|c||}{ lepton-tagged events } \\
\hline $0.6-1.1$ & $1.7 \pm 2.4$ & $0.9 \pm 2.4$ & $60.8 \pm 8.6$ & $55.3 \pm 8.2$ & $0.050 \pm 0.10$ \\
$1.1-1.5$ & $0.4 \pm 2.0$ & $-0.4 \pm 2.3$ & $28.1 \pm 6.2$ & $29.2 \pm 6.5$ & $-0.02 \pm 0.16$ \\
$1.5-1.9$ & $3.1 \pm 3.1$ & $3.6 \pm 3.5$ & $22.0 \pm 5.8$ & $25.4 \pm 6.2$ & $-0.07 \pm 0.18$ \\
$1.9-2.3$ & $4.3 \pm 4.3$ & $-2.2 \pm 4.2$ & $12.5 \pm 5.6$ & $6.5 \pm 5.5$ & $0.32 \pm 0.44$ \\
\hline $0.6-2.3$ & $12.6 \pm 6.7$ & $2.3 \pm 6.8$ & $125 \pm 13$ & $119 \pm 14$ & $0.02 \pm 0.08$ \\
\hline \hline \multicolumn{7}{|c||}{ Lepton- untagged events } \\
\hline $0.6-1.1$ & $17.1 \pm 9.3$ & $14.7 \pm 9.7$ & $411 \pm 27$ & $424 \pm 30$ & $-0.02 \pm 0.04$ \\
$1.1-1.5$ & $6.5 \pm 6.0$ & $7.5 \pm 6.7$ & $169 \pm 21$ & $183 \pm 22$ & $-0.04 \pm 0.08$ \\
$1.5-1.9$ & $6.9 \pm 8.1$ & $15.7 \pm 9.8$ & $95 \pm 21$ & $119 \pm 25$ & $-0.11 \pm 0.16$ \\
$1.9-2.3$ & $17 \pm 14$ & $12 \pm 14$ & $101 \pm 30$ & $76 \pm 28$ & $0.14 \pm 0.24$ \\
\hline $0.6-2.3$ & $48 \pm 21$ & $58 \pm 21$ & $776 \pm 51$ & $777 \pm 53$ & $0.00 \pm 0.05$ \\
\hline
\end{tabular}

Table 39: Number of peaking background events and number of peaking signal and background events, for lepton-tagged and untagged events, with $K^{ \pm}$yield increased by $10 \%$.

Then by looking at data we found a $\mathrm{K}^{+}$to $\mathrm{K}_{S}$ ratio of $1.08 \pm 0.08$, and a $\pi^{0}$ to $\pi$ ratio of 1.20. So we reweighted the signal Monte Carlo by a factor of 1.08 for the kaon ratio and 
1.2 for the pion ratio. This reweighting of the Monte Carlo to data gave a $C P$ asymmetry of $-0.003 \pm 0.052$.

We also checked that the $B^{+}\left(K^{+}\right)$decay to $B^{0}\left(K^{0}\right)$ decay (The $B^{0}\left(K^{0}\right)$ decays were not used in the main analysis.) ratio was the same as found earlier for the $\mathrm{K}^{+}$to $\mathrm{K}_{S}$ ratio. We found the $B^{+}\left(K^{+}\right)$decay to $B^{0}\left(K^{0}\right)$ decay ratio to be $0.99 \pm 0.11$, and in good agreement with the previous $\mathrm{K}^{+}$to $\mathrm{K}_{S}$ ratio.

\begin{tabular}{|c|c|c|c|c|c|}
\hline \multirow{2}{*}{$\begin{array}{l}\text { Momentum } \\
(\mathrm{GeV})\end{array}$} & \multicolumn{2}{|c|}{ RUN1 } & \multicolumn{2}{|c|}{ RUN2 } & \multirow[b]{2}{*}{$A_{C P}{ }^{D e t}$} \\
\hline & $\varepsilon\left(K^{+}\right)$ & $\varepsilon\left(K^{-}\right)$ & $\varepsilon\left(K^{+}\right)$ & $\varepsilon\left(K^{-}\right)$ & \\
\hline $0.250-0.500$ & $1.000 \pm 0.014$ & $1.000 \pm 0.014$ & $1.000 \pm 0.013$ & $0.990 \pm 0.013$ & $-0.003 \pm 0.007$ \\
\hline $0.500-0.625$ & $0.935 \pm 0.011$ & $0.907 \pm 0.010$ & $0.918 \pm 0.009$ & $0.877 \pm 0.010$ & $-0.019 \pm 0.005$ \\
\hline $0.625-0.750$ & $0.812 \pm 0.010$ & $0.791 \pm 0.010$ & $0.807 \pm 0.009$ & $0.783 \pm 0.009$ & $-0.014 \pm 0.006$ \\
\hline $0.750-0.875$ & $0.713 \pm 0.010$ & $0.713 \pm 0.010$ & $0.716 \pm 0.008$ & $0.690 \pm 0.009$ & $-0.011 \pm 0.006$ \\
\hline $0.875-1.000$ & $0.807 \pm 0.009$ & $0.771 \pm 0.009$ & $0.798 \pm 0.007$ & $0.786 \pm 0.008$ & $-0.014 \pm 0.005$ \\
\hline $1.000-1.120$ & $0.855 \pm 0.008$ & $0.839 \pm 0.008$ & $0.854 \pm 0.007$ & $0.827 \pm 0.007$ & $-0.013 \pm 0.004$ \\
\hline $1.120-1.250$ & $0.864 \pm 0.008$ & $0.857 \pm 0.007$ & $0.857 \pm 0.006$ & $0.860 \pm 0.006$ & $-0.001 \pm 0.004$ \\
\hline $1.250-1.500$ & $0.884 \pm 0.005$ & $0.875 \pm 0.005$ & $0.881 \pm 0.004$ & $0.865 \pm 0.004$ & $-0.007 \pm 0.003$ \\
\hline $1.500-1.750$ & $0.888 \pm 0.005$ & $0.892 \pm 0.005$ & $0.885 \pm 0.004$ & $0.883 \pm 0.004$ & $0.001 \pm 0.002$ \\
\hline $1.750-2.000$ & $0.888 \pm 0.005$ & $0.885 \pm 0.005$ & $0.891 \pm 0.004$ & $0.884 \pm 0.004$ & $-0.003 \pm 0.003$ \\
\hline $2.000-$ & $0.898 \pm 0.005$ & $0.879 \pm$ & $3 \pm 0.005$ & $0.893 \pm$ & $-0.004 \pm 0.003$ \\
\hline $2.250-2.500$ & $0.878 \pm$ & $0.888 \pm 0$ & $0.878 \pm 0.006$ & $0.881 \pm$ & $0.003 \pm 0.003$ \\
\hline $2.500-2.750$ & $0.879 \pm 0.007$ & $0.861 \pm 0.008$ & $0.872 \pm 0.006$ & $0.851 \pm$ & $-0.011 \pm 0.004$ \\
\hline 2.750 & $0.857 \pm 0.009$ & $0.829 \pm$ & $0.845 \pm 0.008$ & $0.835 \pm$ & $-0.011 \pm 0.005$ \\
\hline $3.000-$ & $0.810 \pm 0.011$ & $0.792 \pm$ & $0.815 \pm 0.009$ & $0.800 \pm$ & $-0.010 \pm 0.006$ \\
\hline $3.250-3.500$ & $0.763 \pm 0.014$ & $0.749 \pm 0.014$ & $0.763 \pm 0.012$ & $0.732 \pm 0.012$ & $-0.016 \pm 0.009$ \\
\hline $3.500-3.750$ & $0.698 \pm 0.017$ & $0.680 \pm 0.017$ & $0.708 \pm 0.014$ & $0.701 \pm 0.014$ & $-0.008 \pm 0.011$ \\
\hline $3.750-4.000$ & $0.623 \pm 0.020$ & $0.611 \pm 0.021$ & $0.638 \pm 0.016$ & $0.616 \pm 0.017$ & $-0.015 \pm 0.015$ \\
\hline $4.000-5.000$ & $0.458 \pm 0.015$ & $0.431 \pm 0.015$ & $0.440 \pm 0.013$ & $0.437 \pm 0.013$ & $-0.014 \pm 0.016$ \\
\hline $0.250-5.000$ & $0.848 \pm 0.002$ & $0.834 \pm 0.002$ & $0.848 \pm 0.001$ & $0.834 \pm 0.001$ & $-0.0061 \pm 0.0009$ \\
\hline
\end{tabular}

Table 40: Kaon efficiency $\varepsilon\left(K^{+}\right)$and $\varepsilon\left(K^{-}\right)$for positively and negatively charged kaons during RUN1 and RUN2 as a function of the kaon momentum and the resulting detector efficiency $C P$ asymmetry. The kaon efficiency is obtained from the official PID tables. The apparent $C P$ asymmetry is defined as $A_{C P}{ }^{D e t}=\frac{\varepsilon\left(K^{-}\right)-\varepsilon\left(K^{+}\right)}{\varepsilon\left(K^{-}\right)+\varepsilon\left(K^{+}\right)}$. 


\begin{tabular}{|l||c|c|}
\hline Categories & $A_{C P}{ }^{\text {Det }}{ }_{\text {tag. }}$ & $A_{C P}{ }^{\text {Det }}{ }_{\text {untag. }}$ \\
\hline$M_{X s}=0.6-1.1 \mathrm{GeV}$ & $0.013 \pm 0.015$ & $0.000 \pm 0.005$ \\
$M_{X s}=1.1-1.5 \mathrm{GeV}$ & $-0.027 \pm 0.048$ & $-0.014 \pm 0.018$ \\
$M_{X s}=1.5-1.9 \mathrm{GeV}$ & $0.040 \pm 0.055$ & $0.041 \pm 0.021$ \\
$M_{X s}=1.9-2.3 \mathrm{GeV}$ & $-0.029 \pm 0.064$ & $0.035 \pm 0.024$ \\
\hline$M_{X s}=0.6-2.3 \mathrm{GeV}$ & $0.009 \pm 0.013$ & $0.003 \pm 0.005$ \\
\hline$B^{0}$ & $0.018 \pm 0.018$ & $0.002 \pm 0.006$ \\
$B^{ \pm}\left(K^{ \pm}\right)$ & $-0.025 \pm 0.028$ & $-0.027 \pm 0.010$ \\
$B^{ \pm}\left(K^{0}\right)$ & $0.000 \pm 0.031$ & $0.011 \pm 0.011$ \\
\hline
\end{tabular}

Table 41: Apparent $C P$ asymmetry in reconstructed Monte Carlo signal events due to the detector efficiency bias as a function of the hadronic mass bins for lepton-tagged $\left(A_{C P}{ }^{D e t}\right.$ tag.) and untagged events $\left(A_{C P}{ }^{\text {Det }}\right.$ untag. $)$.

\begin{tabular}{|l||c|c|}
\hline$M_{X s}(\mathrm{GeV})$ & $A_{C P}{ }^{\text {Det }}$ tag. & $A_{C P}^{\text {Det }}$ untag. \\
\hline $0.6-1.1$ & $-0.002 \pm 0.015$ & $-0.014 \pm 0.006$ \\
$1.1-1.5$ & $-0.043 \pm 0.047$ & $-0.031 \pm 0.018$ \\
$1.5-1.9$ & $0.023 \pm 0.055$ & $0.025 \pm 0.021$ \\
$1.9-2.3$ & $-0.046 \pm 0.063$ & $0.017 \pm 0.024$ \\
\hline $0.6-2.3$ & $-0.005 \pm 0.013$ & $-0.012 \pm 0.005$ \\
\hline$B^{0}$ & $0.012 \pm 0.018$ & $-0.003 \pm 0.006$ \\
$B^{ \pm}\left(K^{ \pm}\right)$ & $-0.032 \pm 0.028$ & $-0.034 \pm 0.010$ \\
$B^{ \pm}\left(K^{0}\right)$ & $-0.047 \pm 0.030$ & $-0.037 \pm 0.011$ \\
\hline
\end{tabular}

Table 42: Apparent $C P$ asymmetry in reconstructed Monte Carlo signal events due to the detector efficiency bias as a function of the hadronic mass bins for lepton-tagged $\left(A_{C P}{ }^{D e t}\right.$ tag. $)$ and untagged events $\left(A_{C P}{ }^{D e t}{ }_{\text {untag. }}\right)$. The signal events are re-weighted according to the $A_{C P}$ found in data events. 


\begin{tabular}{|l||c|c||c|c||c|}
\hline \multicolumn{1}{|l||}{ Momentum Range } \\
$(\mathrm{GeV})$ & \multicolumn{2}{c||}{ RUN1 } & \multicolumn{2}{c||}{ RUN2 } \\
\hline $0.250-0.500$ & $\varepsilon\left(\pi^{+} \rightarrow K^{+}\right)$ & $\varepsilon\left(\pi^{-} \rightarrow K^{-}\right)$ & $\varepsilon\left(\pi^{+} \rightarrow K^{+}\right)$ & $\varepsilon\left(\pi^{-} \rightarrow K^{-}\right)$ & $A_{C P}$ fake \\
$0.500-0.625$ & $0.004 \pm 0.003$ & $0.003 \pm 0.002$ & $0.004 \pm 0.002$ & $0.003 \pm 0.002$ & $-0.0003 \pm 0.0012$ \\
$0.625-0.750$ & $0.020 \pm 0.004$ & $0.011 \pm 0.003$ & $0.011 \pm 0.003$ & $0.008 \pm 0.002$ & $-0.0029 \pm 0.0014$ \\
$0.750-0.875$ & $0.028 \pm 0.004$ & $0.019 \pm 0.003$ & $0.015 \pm 0.002$ & $0.017 \pm 0.003$ & $-0.0007 \pm 0.0014$ \\
$0.875-1.000$ & $0.001 \pm 0.001$ & $0.003 \pm 0.002$ & $0.002 \pm 0.001$ & $0.002 \pm 0.001$ & $0.0004 \pm 0.0007$ \\
$1.000-1.120$ & $0.007 \pm 0.002$ & $0.010 \pm 0.002$ & $0.008 \pm 0.009$ & $0.009 \pm 0.002$ & $0.0011 \pm 0.0010$ \\
$1.120-1.250$ & $0.013 \pm 0.003$ & $0.012 \pm 0.003$ & $0.014 \pm 0.002$ & $0.011 \pm 0.002$ & $-0.0016 \pm 0.0012$ \\
$1.250-1.500$ & $0.019 \pm 0.003$ & $0.018 \pm 0.003$ & $0.017 \pm 0.002$ & $0.018 \pm 0.002$ & $-0.0000 \pm 0.0013$ \\
$1.500-1.750$ & $0.021 \pm 0.002$ & $0.015 \pm 0.002$ & $0.021 \pm 0.002$ & $0.016 \pm 0.002$ & $-0.0029 \pm 0.0010$ \\
$1.750-2.000$ & $0.017 \pm 0.002$ & $0.016 \pm 0.002$ & $0.021 \pm 0.002$ & $0.014 \pm 0.002$ & $-0.0024 \pm 0.0010$ \\
$2.000-2.250$ & $0.018 \pm 0.003$ & $0.020 \pm 0.003$ & $0.020 \pm 0.002$ & $0.017 \pm 0.002$ & $-0.0005 \pm 0.0011$ \\
$2.250-2.500$ & $0.024 \pm 0.003$ & $0.024 \pm 0.003$ & $0.019 \pm 0.002$ & $0.018 \pm 0.002$ & $-0.0003 \pm 0.0013$ \\
$2.500-2.750$ & $0.031 \pm 0.004$ & $0.030 \pm 0.004$ & $0.026 \pm 0.003$ & $0.028 \pm 0.003$ & $0.0002 \pm 0.0017$ \\
$2.750-3.000$ & $0.016 \pm 0.004$ & $0.022 \pm 0.004$ & $0.023 \pm 0.004$ & $0.026 \pm 0.004$ & $0.0026 \pm 0.0018$ \\
$3.000-3.250$ & $0.031 \pm 0.005$ & $0.028 \pm 0.005$ & $0.027 \pm 0.004$ & $0.032 \pm 0.004$ & $0.0009 \pm 0.0023$ \\
$3.250-3.500$ & $0.029 \pm 0.006$ & $0.033 \pm 0.007$ & $0.027 \pm 0.005$ & $0.032 \pm 0.005$ & $0.0031 \pm 0.0028$ \\
$3.500-3.750$ & $0.024 \pm 0.006$ & $0.029 \pm 0.007$ & $0.035 \pm 0.007$ & $0.028 \pm 0.006$ & $-0.0002 \pm 0.0031$ \\
$3.750-4.000$ & $0.046 \pm 0.010$ & $0.047 \pm 0.010$ & $0.041 \pm 0.009$ & $0.033 \pm 0.008$ & $-0.0036 \pm 0.0045$ \\
$4.000-5.000$ & $0.037 \pm 0.011$ & $0.025 \pm 0.010$ & $0.023 \pm 0.008$ & $0.019 \pm 0.007$ & $-0.0052 \pm 0.0044$ \\
\hline $0.250-5.000$ & $0.021 \pm 0.007$ & $0.023 \pm 0.008$ & $0.019 \pm 0.006$ & $0.027 \pm 0.007$ & $0.0054 \pm 0.0034$ \\
\hline
\end{tabular}

Table 43: Pion to Kaon mis-ID rates $\varepsilon\left(\pi^{+} \rightarrow K^{+}\right)$and $\varepsilon\left(\pi^{-} \rightarrow K^{-}\right)$for positively and negatively charged pions during RUN1 and RUN2 as a function of the pion momentum and the resulting fake $C P$ asymmetry. The pion mis-ID rates are obtained from the official PID tables. The fake $C P$ asymmetry is defined as $A_{C P}$ fake $=\frac{\varepsilon\left(\pi^{-} \rightarrow K^{-}\right)-\varepsilon\left(\pi^{+} \rightarrow K^{+}\right)}{\varepsilon\left(K^{-}\right)+\varepsilon\left(K^{+}\right)}$. 


\begin{tabular}{|l|c|c|c|c|}
\hline Final States & Same flavor & Opp. flavor & To mixed-flavor & From mixed-flavor \\
\hline$B^{+} \rightarrow K_{s} \pi^{+} \gamma$ & - & $K_{s} \rightarrow K^{-}$ & - & - \\
$B^{+} \rightarrow K^{+} \pi^{0} \gamma$ & $\pi^{0} \rightarrow \pi^{-}$ & - & - & - \\
$B^{0} \rightarrow K^{+} \pi^{-} \gamma$ & - & $K^{+} \rightarrow K_{s}$ & - & - \\
$B^{0} \rightarrow K_{s} \pi^{0} \gamma$ & - & - & - & $K_{s} \rightarrow K^{ \pm}$ \\
& & & & $\pi^{0} \rightarrow \pi^{ \pm}$ \\
\hline$B^{+} \rightarrow K^{+} \pi^{+} \pi^{-} \gamma$ & $\pi^{+} \rightarrow \pi^{0}$ & $K^{+} \rightarrow K_{s}$ & - & - \\
$B^{+} \rightarrow K_{s} \pi^{+} \pi^{0} \gamma$ & - & $K_{s} \rightarrow K^{-}$ & $\pi^{+, 0} \rightarrow \pi^{0,-}$ & - \\
$B^{+} \rightarrow K^{+} \pi^{0} \pi^{0} \gamma$ & $\pi^{0} \rightarrow \pi^{-}$ & - & $K^{+} \rightarrow K_{s}$ & $K_{s} \rightarrow K^{ \pm}$ \\
$B^{0} \rightarrow K_{s} \pi^{+} \pi^{-} \gamma$ & - & - & - & $\pi^{ \pm} \rightarrow \pi^{0}$ \\
$B^{0} \rightarrow K^{+} \pi^{-} \pi^{0} \gamma$ & $\pi^{0,-} \rightarrow \pi^{+, 0}$ & $K^{+} \rightarrow K_{s}$ & - & $K_{s} \rightarrow K^{ \pm}$ \\
$B^{0} \rightarrow K_{s} \pi^{0} \pi^{0} \gamma$ & - & - & - & $\pi^{0} \rightarrow \pi^{ \pm}$ \\
\hline$B^{+} \rightarrow K_{s} \pi^{+} \pi^{-} \pi^{+} \gamma$ & - & & & - \\
$B^{+} \rightarrow K^{+} \pi^{+} \pi^{-} \pi^{0} \gamma$ & $\pi^{+, 0} \rightarrow \pi^{0,-}$ & $K_{s} \rightarrow K^{-}$ & $\pi^{+} \rightarrow \pi^{0}$ & - \\
$B^{+} \rightarrow K_{s} \pi^{+} \pi^{0} \pi^{0} \gamma$ & - & $K_{s} \rightarrow K^{-}$ & $\pi^{+} \rightarrow \pi^{0}$ & - \\
$B^{0} \rightarrow K^{+} \pi^{-} \pi^{+} \pi^{-} \gamma$ & $\pi^{-} \rightarrow \pi^{0}$ & $K^{+} \rightarrow K_{s}$ & - & $K_{s} \rightarrow K^{ \pm}$ \\
$B^{0} \rightarrow K_{s} \pi^{0} \pi^{+} \pi^{-} \gamma$ & - & - & - & $\pi^{ \pm, 0} \rightarrow \pi^{0, \pm}$ \\
$B^{0} \rightarrow K^{+} \pi^{-} \pi^{0} \pi^{0} \gamma$ & $\pi^{-, 0} \rightarrow \pi^{0,+}$ & $K^{+} \rightarrow K_{s}$ & - & - \\
\hline
\end{tabular}

Table 44: Cross-feed events that can contribute to CP asymmetries due to replacement of a final state particle. The table also includes cases where not mixed-flavors change to mixed-flavors, and vice-versa. 


\begin{tabular}{|c|c|c|c|c|}
\hline Final States & Same flavor & Opposite flavor & To mixed-flavor & From mixed-flavor \\
\hline$B^{+} \rightarrow K_{s} \pi^{+} \gamma$ & $+\pi^{0}$ & - & $+\pi^{-}$ & - \\
\hline$B^{+} \rightarrow K^{+} \pi^{0} \gamma$ & $+\pi^{0},+\pi^{-}$ & - & - & - \\
\hline$B^{0} \rightarrow K^{+} \pi^{-} \gamma$ & $+\pi^{0},+\pi^{+}$ & - & - & - \\
\hline$B^{0} \rightarrow K_{s} \pi^{0} \gamma$ & - & - & $+\pi^{0}$ & $+\pi^{ \pm}$ \\
\hline$B^{+} \rightarrow K^{+} \pi^{+} \pi^{-} \gamma$ & $+\pi^{0},-\pi^{+},+\pi^{-}$ & - & - & - \\
\hline$B^{+} \rightarrow K_{s} \pi^{+} \pi^{0} \gamma$ & $\pm \pi^{0}$ & - & - & $+\pi^{-}$ \\
\hline$B^{+} \rightarrow K^{+} \pi^{0} \pi^{0} \gamma$ & $-\pi^{0},+\pi^{-}$ & - & - & - \\
\hline$B^{0} \rightarrow K_{s} \pi^{+} \pi^{-} \gamma$ & - & - & $+\pi^{0}$ & $\pm \pi^{ \pm}$ \\
\hline$B^{0} \rightarrow K^{+} \pi^{-} \pi^{0} \gamma$ & $\pm \pi^{0},-\pi^{-},+\pi^{+}$ & - & - & - \\
\hline$B^{0} \rightarrow K_{s} \pi^{0} \pi^{0} \gamma$ & - & - & $-\pi^{0}$ & $+\pi^{ \pm}$ \\
\hline$B^{+} \rightarrow K_{s} \pi^{+} \pi^{-} \pi^{+} \gamma$ & - & - & - & $-\pi^{+}$ \\
\hline$B^{+} \rightarrow K^{+} \pi^{+} \pi^{-} \pi^{0} \gamma$ & $-\pi^{+},-\pi^{0}$ & - & - & - \\
\hline$B^{+} \rightarrow K_{s} \pi^{+} \pi^{0} \pi^{0} \gamma$ & $-\pi^{0}$ & 一 & $-\pi^{+}$ & \\
\hline$B^{0} \rightarrow K^{+} \pi^{-} \pi^{+} \pi^{-} \gamma$ & $-\pi^{-}$ & - & - & - \\
\hline$B^{0} \rightarrow K_{s} \pi^{0} \pi^{+} \pi^{-} \gamma$ & - & - & - & $-\pi^{ \pm}$ \\
\hline$B^{0} \rightarrow K^{+} \pi^{-} \pi^{0} \pi^{0} \gamma$ & $-\pi^{0},-\pi^{-}$ & - & - & - \\
\hline
\end{tabular}

Table 45: Cross-feed events that can contribute to $C P$ asymmetries due to the addition or removal of a pion in the final state. Again note that there are cases where not mixed-flavors change to mixed-flavors, and vice-versa.

\begin{tabular}{|c||c|c|}
\hline \multicolumn{3}{|c|}{ Float Argus Shape } \\
$M_{X s}(\mathrm{GeV})$ & $\bar{b}$ events & $b$ events \\
\hline \hline \multicolumn{1}{|c||}{} & Continuum MC events \\
\hline $0.6-1.1$ & $-33.0 \pm 4.0$ & $-31.1 \pm 4.0$ \\
$1.1-1.5$ & $-15.5 \pm 4.9$ & $-13.6 \pm 4.9$ \\
$1.5-1.9$ & $-5.6 \pm 3.6$ & $-2.1 \pm 3.7$ \\
$1.9-2.3$ & $-5.0 \pm 2.6$ & $-4.3 \pm 2.7$ \\
\hline $0.6-2.3$ & $-9.2 \pm 1.8$ & $-10.2 \pm 1.8$ \\
\hline$B^{0}$ & $-14.5 \pm 2.8$ & $-16.9 \pm 2.8$ \\
$B \pm\left(K^{ \pm}\right)$ & $-5.3 \pm 2.9$ & $-3.4 \pm 3.0$ \\
$B^{ \pm}\left(K^{0}\right)$ & $-6.6 \pm 3.6$ & $-9.6 \pm 3.7$ \\
\hline
\end{tabular}

Table 46: Comparison of $b$ and $\bar{b}$ Argus parameters for a fit to the lepton-untagged continuum MC events. 


\begin{tabular}{|c||c|c||c|c||c|c|}
\hline \multicolumn{1}{|c||}{$M_{X s}(\mathrm{GeV})$} & \multicolumn{9}{c|}{$\sigma(\mathrm{MeV})$} & \multicolumn{3}{c|}{$n$} \\
& $\bar{b}$ events & $b$ events & $\bar{b}$ events & $b$ events & $\bar{b}$ events & $b$ events \\
\hline \hline \multicolumn{1}{|c||}{} & \multicolumn{7}{c|}{ Signal MC events } \\
\hline $0.6-1.1$ & $2.73 \pm 0.11$ & $2.74 \pm 0.12$ & $2.3 \pm 0.5$ & $2.3 \pm 0.5$ & $2.39 \pm 1.48$ & $2.64 \pm 1.72$ \\
$1.1-1.5$ & $2.80 \pm 0.17$ & $2.78 \pm 0.17$ & $2.1 \pm 0.3$ & $2.1 \pm 0.3$ & $2.01 \pm 1.01$ & $1.78 \pm 0.86$ \\
$1.5-1.9$ & $2.89 \pm 0.23$ & $2.82 \pm 0.22$ & $2.6 \pm 0.6$ & $2.1 \pm 0.4$ & $0.68 \pm 0.76$ & $1.37 \pm 0.71$ \\
$1.9-2.3$ & $2.94 \pm 0.42$ & $2.84 \pm 0.29$ & $2.6 \pm 2.2$ & $2.1 \pm 0.6$ & $0.40 \pm 0.91$ & $1.12 \pm 0.87$ \\
\hline $0.6-2.3$ & $2.80 \pm 0.09$ & $2.78 \pm 0.10$ & $2.4 \pm 0.3$ & $2.2 \pm 0.3$ & $1.32 \pm 0.56$ & $1.68 \pm 0.69$ \\
\hline$B^{0}$ & $2.74 \pm 0.19$ & $2.75 \pm 0.10$ & $2.5 \pm 0.3$ & $2.5 \pm 0.3$ & $1.46 \pm 0.70$ & $1.26 \pm 0.59$ \\
$B^{ \pm}\left(K^{ \pm}\right)$ & $3.04 \pm 0.19$ & $2.93 \pm 0.21$ & $2.2 \pm 0.2$ & $1.7 \pm 0.4$ & $1.28 \pm 0.30$ & $2.27 \pm 1.26$ \\
$B^{ \pm}\left(K^{0}\right)$ & $2.70 \pm 0.19$ & $2.67 \pm 0.18$ & $2.5 \pm 0.2$ & $2.2 \pm 0.4$ & $1.15 \pm 0.36$ & $1.70 \pm 0.95$ \\
\hline
\end{tabular}

Table 47: Comparison of $\bar{b}$ and $b$ floated Crystal Ball parameters for lepton-untagged signal $\mathrm{MC}$ events (normally fixed to a weighted average).

\begin{tabular}{|c||c|c||c|c|}
\hline \multicolumn{1}{|c||}{} & \multicolumn{4}{c|}{ Float Argus Shape } \\
\multicolumn{1}{|c||}{$M_{X s}(\mathrm{GeV})$} & $\bar{b}$ events & $b$ events & $\bar{b}$ events & $b$ events \\
\hline \hline \multicolumn{1}{|c||}{} & \multicolumn{3}{c|}{ cross-feed and $B \bar{B}$ MC events } \\
\hline $0.6-1.1$ & $-132 \pm 14$ & $-133 \pm 14$ & $173 \pm 15$ & $176 \pm 15$ \\
$1.1-1.5$ & $-79 \pm 17$ & $-72 \pm 18$ & $95 \pm 11$ & $89 \pm 11$ \\
$1.5-1.9$ & $-45 \pm 10$ & $-50 \pm 27$ & $263 \pm 18$ & $271 \pm 18$ \\
$1.9-2.3$ & $-37 \pm 6$ & $-32 \pm 6$ & $692 \pm 29$ & $680 \pm 28$ \\
\hline $0.6-2.3$ & $-53 \pm 5$ & $-51 \pm 5$ & $1214 \pm 39$ & $1205 \pm 39$ \\
\hline$B^{0}$ & $-56.7 \pm 7.3$ & $-56.8 \pm 7.4$ & $543 \pm 27$ & $524 \pm 26$ \\
$B{ }^{ \pm}\left(K^{ \pm}\right)$ & $-48.8 \pm 8.4$ & $-46.0 \pm 8.2$ & $402 \pm 23$ & $417 \pm 23$ \\
$B^{ \pm}\left(K^{0}\right)$ & $-52.5 \pm 10.0$ & $-46.4 \pm 10.0$ & $267 \pm 18$ & $263 \pm 18$ \\
\hline
\end{tabular}

Table 48: Comparison of $b$ and $\bar{b}$ Argus parameters and corresponding yields for a fit to the lepton-untagged cross-feed and $B \bar{B}$ MC events. 


\section{E Nuclear Cross-sections for Charged Kaons}

Kaons that interact before the DCH could be undetected. The difference in the cross-section between $K^{+}$and $K^{-}$(see Figures 43 and 44, respectively, for their cross-sections) can be the source of a fake charge asymmetry. This effect could be not completely taken into account by the tracking and PID tables, the first because it is based on a pion sample, the second because they already assume the existence of a track.

In order to estimate the size of the possible asymmetry, we use the simple model described in $\mathrm{BAD} \# 239^{8}$. It is based on the cross-section for the kaon nuclear interaction as a function of the kaon momentum and uses a simplified material model before the DCH.

Table 49 shows the $C P$ asymmetry we find as a function of the kaon momentum. The

errors on these asymmetry predictions are around $0.1 \%$ above $1 \mathrm{GeV}, 0.2 \%$ between 0.6 and $1.0 \mathrm{GeV}$, and $0.5 \%$ below $0.6 \mathrm{GeV}$. On top of these errors we need to add a small factor due to the incorrect modeling of the detector.

From Table 49 we can see that the $A_{C P}$ numbers are a little too low to account for the $C P$ asymmetries in the data below $1 \mathrm{GeV}$, but they certainly go in the right direction.

\footnotetext{
${ }^{8}$ Internal $B A B A R$ document
} 
39. Plots of cross sections and related quantities $\quad 010001-15$
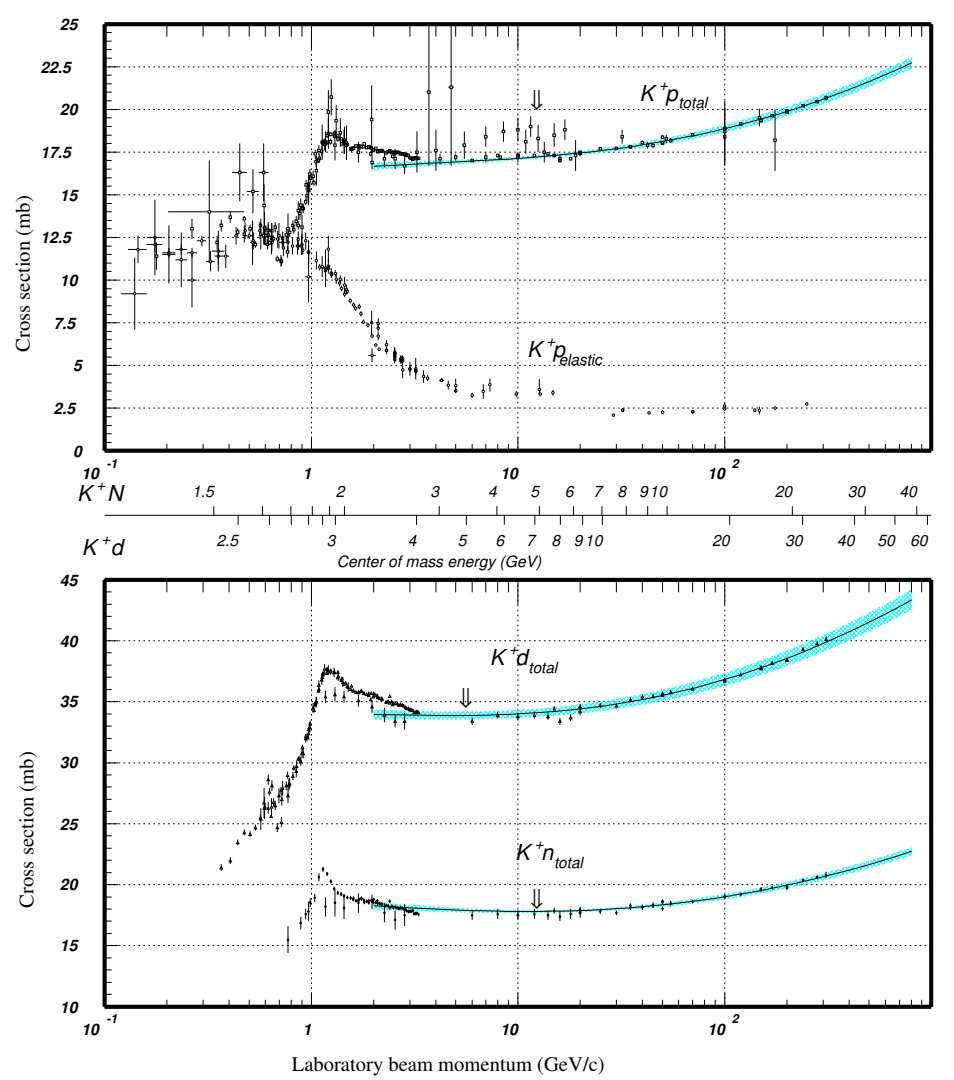

Figure 39.16: Total and elastic cross sections for $K^{+} p$ and total cross sections for $K^{+} d$ and $K^{+} n$ collisions as a funchttp://pdg.lbl.gov//xsect/contents.html (Courtesy of the COMPAS Group, IHEP, Protvino, Russia, August 2001.)

Figure 43: Total and elastic collision cross-section for $K^{+} p, K^{+} d$ (total only) and $K^{+} n$ as a function of the laboratory beam momentum and total center-of-mass energy. 
010001-14 39. Plots of cross sections and related quantities
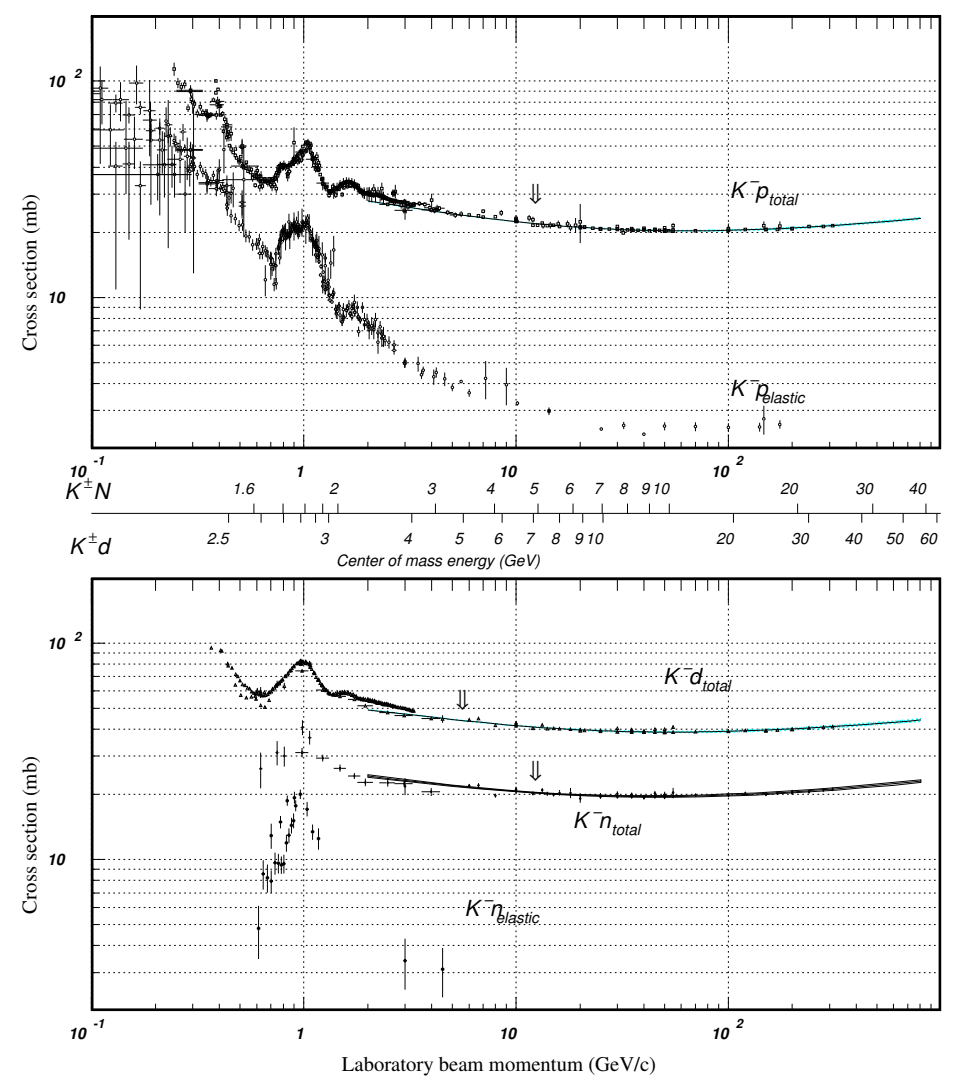

Figure 39.15: Total and elastic cross sections for $K^{-} p$ and $K^{-} d$ (total only), and $K^{-} n$ collisions as a function of laboratory beam momentum (Courtesy of the COMPAS Group, IHEP, Protvino, Russia, August 2001.)

Figure 44: Total and elastic collision cross-section for $K^{-} p, K^{-} d$ (total only) and $K^{-} n$ as a function of the laboratory beam momentum and total centre-of-mass energy. 


\begin{tabular}{|l||c|c|c|c|c|}
\hline \multicolumn{1}{|c||}{} & \multicolumn{2}{c|}{$K^{+}$} & \multicolumn{2}{c|}{$K^{-}$} & \\
$\mathrm{P}_{K}(\mathrm{GeV})$ & $\sigma(m b)$ & $90^{\circ}(\%)$ & $\sigma(m b)$ & $90 \%)$ & $A_{C P}$ \\
\hline $0.4-0.6$ & 15 & 0.9 & 50 & 3.0 & $\sim-0.015$ \\
$0.6-0.8$ & 13.8 & 0.81 & 32.4 & 1.90 & -0.0082 \\
$0.8-1.0$ & 16.0 & 0.94 & 37.9 & 2.22 & -0.0096 \\
$1.0-1.2$ & 18.8 & 1.10 & 42.4 & 2.49 & -0.0104 \\
$1.2-1.4$ & 19.1 & 1.12 & 30.9 & 1.81 & -0.0052 \\
$1.4-1.6$ & 18.4 & 1.08 & 29.6 & 1.74 & -0.0050 \\
$1.6-1.8$ & 18.3 & 1.07 & 28.8 & 1.69 & -0.0047 \\
$1.8-2.0$ & 18.2 & 1.07 & 26.8 & 1.57 & -0.0038 \\
\hline
\end{tabular}

Table 49: $C P$ asymmetry (last column) we find as a function of the kaon momentum (first column). The other columns show the details needed to extract the $A_{C P}$ : the second and the fourth columns show the kaon cross-section while the third and fifth columns show the percentage of lost kaons due to the nuclear interaction at $90^{\circ}$. 


\section{References}

[1] S. Novaes, hep-ph/0001283 (2000).

[2] K. Hagiwara et al. (Particle Data Group), Phys. Rev. D66, 349 (2002).

[3] K. Hagiwara et al. (Particle Data Group), Phys. Rev. D66, 281 (2002).

[4] H. E. Haber and G. L. Kane, Phys. Rep. 117, 75 (1985).

[5] K. Hagiwara et al. (Particle Data Group), Phys. Rev. D66, 98 (2002).

[6] S. Weinberg, Phys. Rev. Lett. 19, 1264 (1967).

[7] R. Barate et al., Phys. Lett. B565, 61 (2003).

[8] D. Abbaneo et al., hep-ex/0312023 (2003).

[9] E. Witten, Phys. Lett. B105, 267 (1981).

[10] M. Spira and P. Zerwas, hep-ph/9803257 (1998).

[11] W. Pauli, I1 Nuovo Cimento 6, 204 (1957).

[12] A. Alavi-Harati et al., Phys. Rev. D67, 012005 (2003).

[13] M. Kobayashi and T. Maskawa, Prog. Theor. Phys. 49, 652 (1973).

[14] K. Hagiwara et al. (Particle Data Group), Phys. Rev. D66, 113 (2002).

[15] L. Wolfenstein, Phys. Rev. Lett. 51, 1945 (1983)

[16] J. Christensen, J. Cronin, V. Fitch, R. Turlay, Phys. Rev. Lett. 13, 138 (1964).

[17] K. Hagiwara et al. (Particle Data Group), Phys. Rev. D66, 559 (2002). 
[18] A. Lai et al., Euro. Phys. Jour. C22, 231 (2001).

[19] R. Mohapatra, J. Pati, L. Wolfenstein, Phys. Rev. D11, 3319 (1975).

[20] K. Hagiwara et al. (Particle Data Group), Phys. Rev. D66, 654 (2002).

[21] K. Lingel, T. Skwarnicki, J. Smith, hep-ex/9804015, (1998).

[22] S. Glashow, J. Iliopoulos, L. Maiani, Phys. Rev. D2, 1285/1292 (1970).

[23] T. Hurth, E. Lunghi, W. Porod, hep-ph/0310282 (2003).

[24] G. Buchalla, A. Buras, M.Lautenbacher, Rev. Mod. Phys. 68, 1125 (1996).

[25] A. Kagan and M. Neubert, Phys. Rev. D58, 094012 (1998).

[26] T. E. Coan et al., Phys. Rev. Lett. 86, 5661 (2001).

[27] S. Nishida et al., hep-ex/0308038, (2003).

[28] A. Kagan and M. Neubert, Euro. Phys. Jour. 7, 5 (1999).

[29] T. Sjostrand, "PYTHIA 5.7 and JETSET 7.4: Physics and Manual", CERN-TH-711293-REV, (1995).

[30] T. Hurth, E.Lunghi, W. Porod, hep-ph/0312260 (2003).

[31] B. Aubert et al., Phys. Rev. Lett. 89, 201802 (2002).

[32] G. C. Fox and S. Wolfram, Nucl. Phys. B149, 413 (1970).

[33] R. Reed and R. Marks II, Neural Smithing, MIT Press, (1999). 
[34] A. Zell, N. Mache, R. Hubner, G. Mamier, M. Vogt, K. Herrmann, M. Schmalzl, T. Sommer, A. Hatzigeorgiou, S. Doring, and D. Posselt, "SNNS : Stuttgart Neural Network Simulator," Tech. Rep. 3/93, Institute for Parallel and Distributed High Performance Systems, University of Stuutgart, Fed. Rep. of Germany, 1993.

[35] H. Albrecht et al., Phys. Lett. B185, 218 (1987).

[36] E. Bloom and C. Peck, Ann. Rev. Nucl. Part. Sci. 33, 143 (1983).

[37] D. Wright, private communication.

[38] B. Aubert et al., Phys. Rev. Lett. 93, 021804 (2004).

[39] K. Kiers, A. Soni, G. Wu, Phys. Rev. D62, 116004 (2000).

[40] Y. Keum, T. Nihei, Y. Okada, Y. Shimizu, Phys. Lett. B460, 333 (1999).

[41] M. Aoki, G. Cho, N. Oshimo, Phys. Rev D60, 035004 (1999).

[42] H. Asatryan and H. Asatrian, Phys. Lett. B460, 148 (1999).

[43] C. Chua, X. He, W. Hu, Phys. Rev. D60, 014003 (1999).

[44] Y. Kim, P. Ko, J. Lee, Nucl. Phys. B544, 64 (1999).

[45] E. Chun, K. Hwang, J. Lee, Phys. Rev. D62, 076006 (2000). 